QA: QA

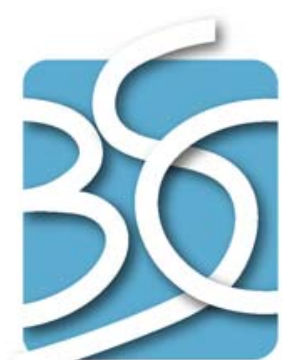

BECHTEL SAIC COMPANYLC
ANL-NBS-HS-000077 REV 00

July 2006

\title{
Data Analysis for Infiltration Modeling: Technical Evaluation of Previous Soil Depth Estimation Methods and Development of Alternate Parameter Values
}

Prepared for:

U.S. Department of Energy

Office of Civilian Radioactive Waste Management

Office of Repository Development

1551 Hillshire Drive

Las Vegas, Nevada 89134-6321

Prepared by:

Bechtel SAIC Company, LLC

1180 Town Center Drive

Las Vegas, Nevada 89144

Under Contract Number

DE-AC28-01RW12101 


\section{DISCLAIMER}

This report was prepared as an account of work sponsored by an agency of the United States Government. Neither the United States Government nor any agency thereof, nor any of their employees, nor any of their contractors, subcontractors or their employees, makes any warranty, express or implied, or assumes any legal liability or responsibility for the accuracy, completeness, or any third party's use or the results of such use of any information, apparatus, product, or process disclosed, or represents that its use would not infringe privately owned rights. Reference herein to any specific commercial product, process, or service by trade name, trademark, manufacturer, or otherwise, does not necessarily constitute or imply its endorsement, recommendation, or favoring by the United States Government or any agency thereof or its contractors or subcontractors. The views and opinions of authors expressed herein do not necessarily state or reflect those of the United States Government or any agency thereof. 
QA: QA

Data Analysis for Infiltration Modeling: Technical Evaluation of Previous Soil Depth Estimation Methods and Development of Alternate Parameter Values

ANL-NBS-HS-000077 REV 00

July 2006 


\section{ACKNOWLEDGMENTS}

This document was developed through the contributions of Terry A. Grant who performed the analysis, created the spreadsheets, and wrote nearly all of the original drafts of the analysis. Tim Vogt provided assistance with creation of many of the maps presented in this analysis. Sharad Khamamkar provided assistance with the checking of the data sets presented in this analysis. 


\section{INTENTIONALLY LEFT BLANK}




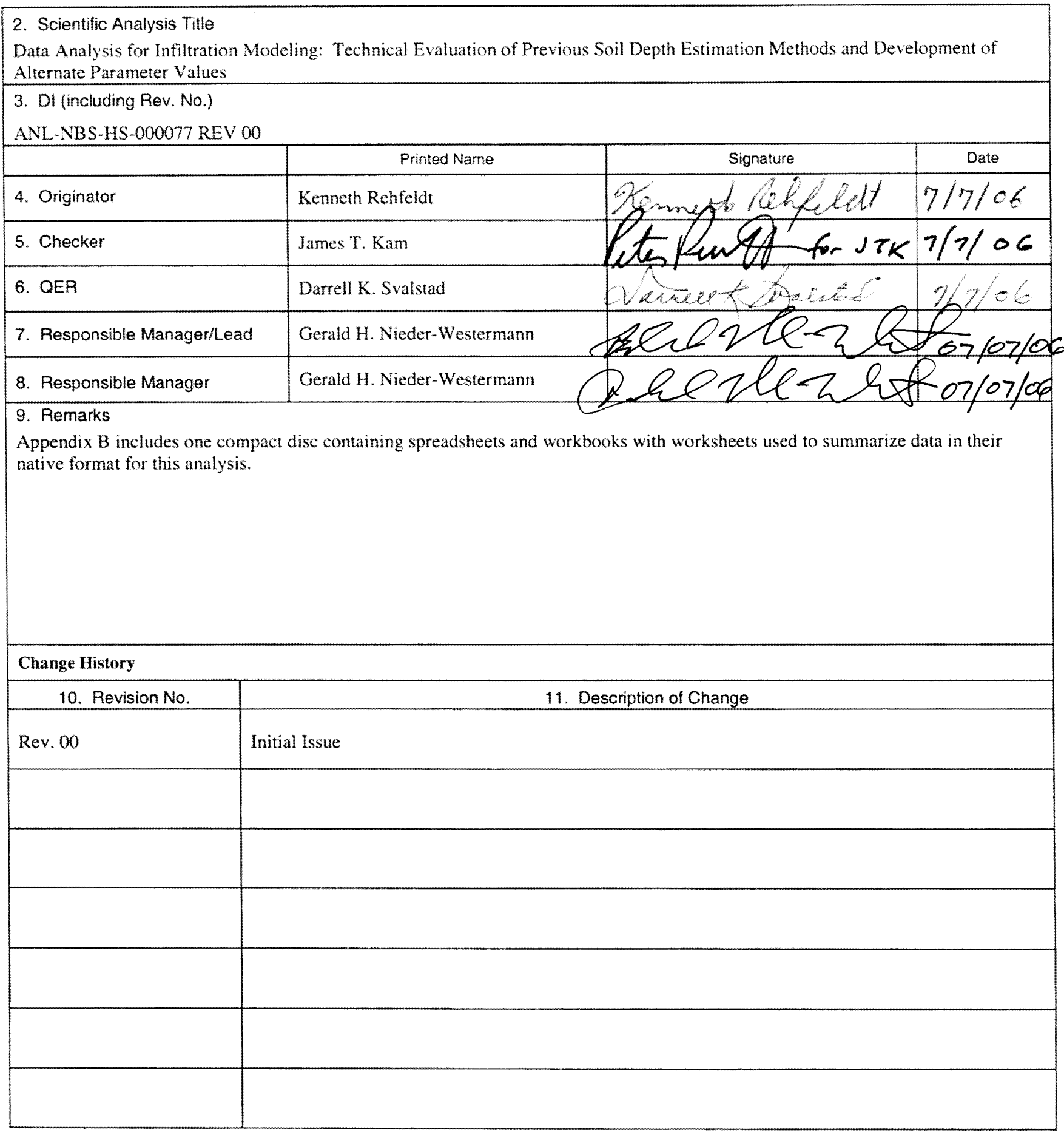




\section{CONTENTS}

Page

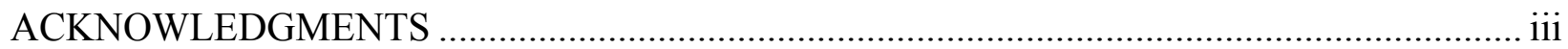

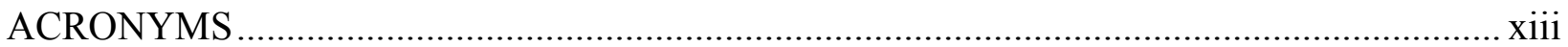

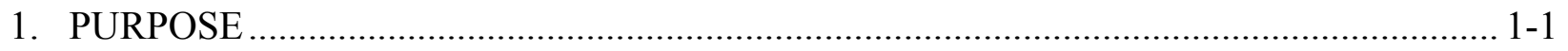

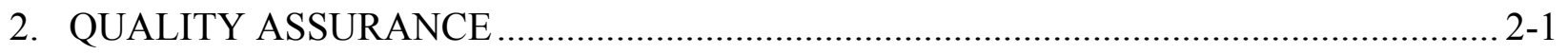

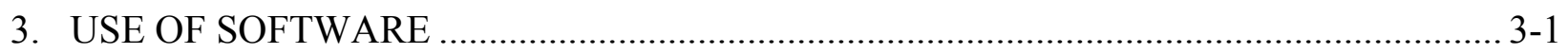

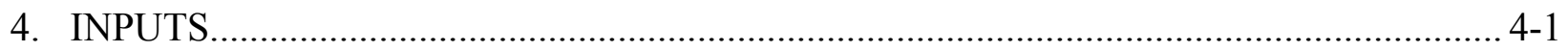

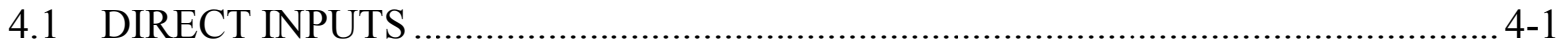

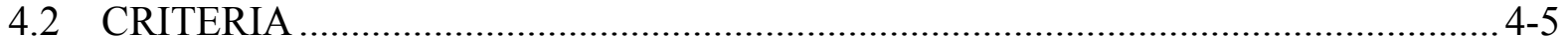

4.3 CODES, STANDARDS, AND REGULATIONS .................................................... 4-6

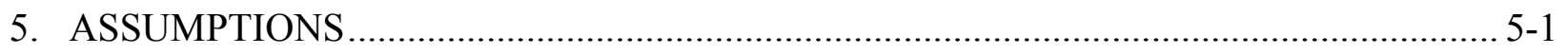

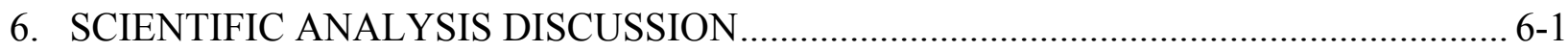

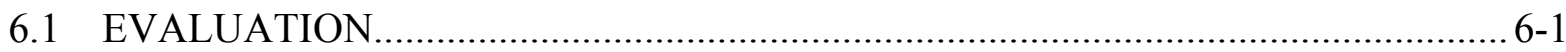

6.1.1 Background Information and Evaluation of SOILMAP6.INP ......................... 6-1

6.1.2 Evaluation of SOILMAP6.INP ............................................................. 6-3

6.2 ALTERNATE SOIL DEPTH EVALUATION …….......................................... 6-22

6.2.1 Summary and Evaluation of Soil Depth Data................................................. 6-23

6.2.2 Uncertainty in Estimating Soil Depth Parameters for Modeling ..................... 6-41

6.2.3 Intended Use of Output and Limitations......................................................... 6-44

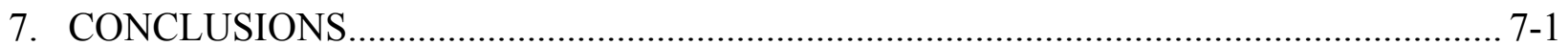

7.1 TECHNICAL ADEQUACY OF THE EXISTING SOIL DEPTH FILE ...................... 7-1

7.2 CONCLUSIONS BASED ON THE SUMMARY OF EXISTING DATA..................... 7-2

7.3 SUMMARY OF THIS ANALYSIS ………………..............................................

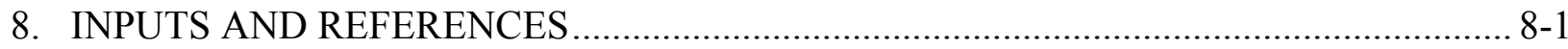

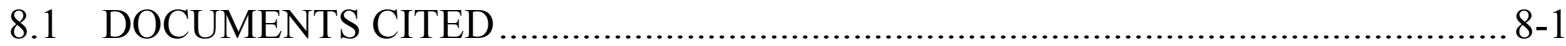

8.2 CODES, STANDARDS, REGULATIONS, AND PROCEDURES ........................... 8-4

8.3 SOURCE DATA, LISTED BY DATA TRACKING NUMBER ………………......... 8-4

8.4 OUTPUT DATA, LISTED BY DATA TRACKING NUMBER …………............... 8-9

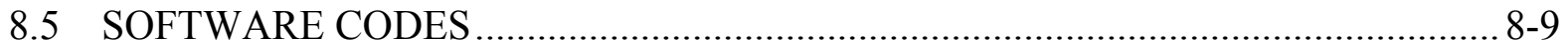

APPENDIX A - BOREHOLES AND SURFICIAL DEPOSIT DEPTH

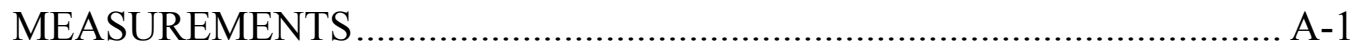

APPENDIX B - ELECTRONIC SPREADSHEETS AND WORKBOOK-WORKSHEETS FOR CALCULATIONS PRESENTED IN THE ANALYSIS 
Data Analysis for Infiltration Modeling: Technical Evaluation of Previous Soil Depth Estimation Methods and Development of Alternate Parameter Values

INTENTIONALLY LEFT BLANK 


\section{FIGURES}

Page

6-1. Representation of the Digital Soil Map Using SOILMAP6.INP ...................................... 6-4

6-2. Surficial Deposit Units Grouped into Depth Classes...................................................... 6-5

6-3. Final Soil Depth Codes in SOILMAP6.INP ............................................................... 6-6

6-4. Comparison of Field Measurements to Depths Calculated Using Equation $\mathrm{D}=0.013 \times(10-\mathrm{S})+0.4$ for $10<\mathrm{S}<40$ Degrees ................................................... 6-11

6-5. Comparison of Field Measurements to Depths Calculated Using Equation $\mathrm{D}=0.03 \times \mathrm{S}+0.1$ for $\mathrm{S} \leq 10$ Degrees.

6-6. Enlargement of the Area around the Prow Showing Soil Depth Values from

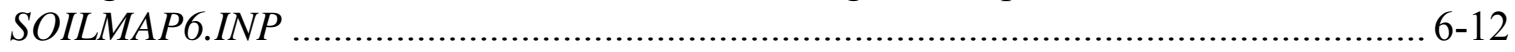

6-7. Topographic Map for the Area Covered in Figure 6-6 ……....................................... 6-13

6-8. Comparison of SOILMAP6.INP Calculated Depths to Borehole Actual Measured Depths .............................................................................................. 6-15

6-9. Area of Densest Borehole Coverage (Enlarged)........................................................ 6-16

6-10. Plot Showing the Relationship between the Soil Depth Measured in Boreholes and the Predicted Soil Depth for the Corresponding Grid Cell in SOILMAP6.INP ...... 6-17

6-11. Data of Borehole Soil Depth Near the Proposed Waste Handling Building (Midway Valley); Projected to an East-West Line

6-12. Graphical Representation of Depth Classes Used for Alternate Soil Depth Evaluation $6-25$

6-13. Distribution of Soil Depths from Surface Field Measurements for Soil Depth Class 4 (Shallow Soils)

6-14. Probability Plot of the $\log _{e}$ Transformed Data Shown in Figure 6-13 ....................... 6-30

6-15. Distribution of Soil Depths from Boreholes and Surface Field Measurements for Soil Depth Class 3 (Intermediate Depth Soils).....

6-16. Probability Plot of the Data Listed in Table 6-7

6-17. Distribution of Soil Depths in Qualified and Unqualified Boreholes for Soil Depth Class 2 (Moderately Deep Soils)

6-18. Probability Plot of the Qualified Data Shown in Figure 6-17 6-36

6-19. Distribution of Soil Depths in Qualified and Unqualified Boreholes for Soil Depth Class 1 (Very Deep Soils).

7-1. Comparison of Values Calculated for Soil Depth Code 1 in SOILMAP6.INP and Soil Depth Class 4 
Data Analysis for Infiltration Modeling: Technical Evaluation of Previous Soil Depth Estimation Methods and Development of Alternate Parameter Values

INTENTIONALLY LEFT BLANK 


\section{TABLES}

Page

4-1. Direct Input Data........................................................................................... 4-2

6-1. Explanation of Soil Depth Codes Used in SOILMAP6.INP ...................................... 6-2

6-2. Classification Scheme Used in the Digital Soil Map for Surficial

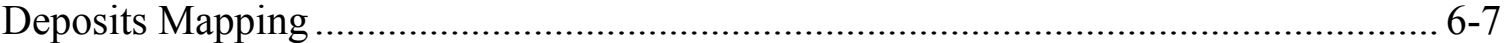

6-3. Comparison of Measured Soil Thickness with Calculated Thickness......................... 6-10

6-4. Comparison between Surficial Deposits Thickness Measured in Boreholes and

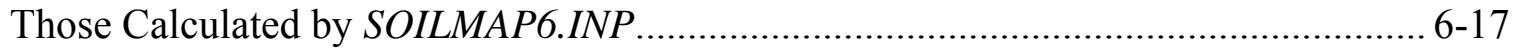

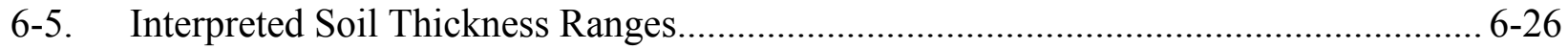

6-6. Field Measurements of Soil Depth for Soil Depth Class 4 ...................................... 6-28

6-7. Field Measurements and Qualified Borehole Depths Used to Calculate Depth

Distributions and Unqualified Borehole Depths Used for Corroboration for Soil

Depth Class 3

6-8. Qualified Borehole Depths Used to Calculate Depth Distribution and Unqualified Borehole Depths Used for Corroboration for Soil Depth Class 2 .............................. 6-36

6-9. Qualified Borehole Soil Depths Used to Determine the Depth Distribution Limits and Unqualified Borehole Soil Depths for Corroboration for Soil Depth Class 1 ....... 6-40

6-10. Summary of Recommended Distributions for Soil Depth Classes Used in Alternate Evaluation

7-1. Mapping of Yucca Mountain Review Plan Acceptance Criteria and Soil Depth Summary

A-1. Boreholes and Surficial Deposit Depth Measurements 
Data Analysis for Infiltration Modeling: Technical Evaluation of Previous Soil Depth Estimation Methods and Development of Alternate Parameter Values

INTENTIONALLY LEFT BLANK 
Data Analysis for Infiltration Modeling: Technical Evaluation of Previous Soil Depth Estimation Methods and Development of Alternate Parameter Values

\section{ACRONYMS}

ACC accession number

DTN data tracking number

GFM geologic framework model

RIS Records Information System

TDMS Technical Data Management System 
Data Analysis for Infiltration Modeling: Technical Evaluation of Previous Soil Depth Estimation Methods and Development of Alternate Parameter Values

INTENTIONALLY LEFT BLANK 


\section{PURPOSE}

The purpose of this analysis is to evaluate the reasonableness of the approach and the results used to generate surficial deposits (soil) depth information in input file SOILMAP6.INP (USGS 2000 [DIRS 175858], folder Soilmap6).

In this analysis, soil refers to unconsolidated surficial deposits that range in composition from talus accumulations of cobbles and boulders to fine-grained eolian deposits. SOILMAP6.INP data were used in support of the infiltration model (BSC 2004 [DIRS 170007], Sections 6.6.2, 6.6.3, and 6.7.1, and Appendices B, F, and G). The evaluation also considers the utility of SOILMAP6.INP (USGS 2000 [DIRS 175858], folder Soilmap6) for use in a future version of the infiltration model. SOILMAP6.INP assigns a soil type and depth to each $30 \times 30 \mathrm{~m}$ grid cell in the model area. Soil type data are used to assign hydrologic and physical properties to surficial deposits for each grid cell. Soil depth data are used directly in the infiltration model for the Yucca Mountain repository.

Through this analysis, SOILMAP6.INP (USGS 2000 [DIRS 175858], folder Soilmap6) data are considered acceptable for current and previous versions of the infiltration model (BSC 2004 [DIRS 170007]); USGS 2000 [DIRS 123650]; USGS 2001 [DIRS 154674]; USGS 2001 [DIRS 160355]; USGS 2003 [DIRS 166518]). They are determined to be not suitable for use in a future infiltration model. The available data are reviewed herein and alternate soil depth parameter values are recommended as part of the analysis for future infiltration modeling described in Technical Work Plan for: Infiltration Model Assessment, Revision, and Analyses of Downstream Impacts (BSC 2006 [DIRS 176107]). Alternate soil depth parameter values are intended for the limited purpose of supporting these revised infiltration modeling calculations.

The evaluation of SOILMAP6.INP (USGS 2000 [DIRS 175858], folder Soilmap6) represents a work scope element of the technical work plan (BSC 2006 [DIRS 176107], Section 1.1.2, Task 1). Objectives of the work scope element are to:

- Address issues related to technical inadequacies in data and references associated with data collection or reduction that were used in the infiltration model report (BSC 2004 [DIRS 170007])

- Evaluate the technical adequacy of the data and their references (BSC 2004 [DIRS 170007]), and compile a list of issues that require updating or correction

- Evaluate data and records, found to have issues, to identify a resolution plan and to identify, as necessary, other potential sources of data, qualify existing data, and reperform data reduction steps, and to document the evaluation and resolution in accordance with LP-SIII.9Q-BSC, Scientific Analysis. 
This analysis does not deviate from the applicable technical work plan and work scope elements (BSC 2006 [DIRS 176107], Section 1.1.2, Task 1). The principal criteria related to the evaluation of SOILMAP6.INP (USGS 2000 [DIRS 175858], folder Soilmap6) are:

- Review the transparency and traceability of the methods used to generate the file and review the adequacy of these methods

- Review the adequacy and qualification status of the source data used to generate the file

- Review any corroborating or contrary data that might exist that were not used in developing the file. 


\section{QUALITY ASSURANCE}

Development of this analysis and its supporting calculation activities are subject to the Yucca Mountain Project quality assurance program, as indicated in the technical work plan (BSC 2006 [DIRS 176107], Section 8.1), using approved quality assurance procedures (BSC 2006 [DIRS 176107], Section 4.1) to conduct and document the activities. The technical work plan also identifies the methods used to control the electronic management of data (BSC 2006 [DIRS 176107], Section 8.4). Calculations herein were conducted and documented following LP-SIII.9Q-BSC.

This analysis examines the properties of the upper natural barrier - surficial soils, classified as "Safety Category" in Q-List (BSC 2005 [DIRS 175539], Appendix A), because they are important to waste isolation as defined in LS-PRO-0203, Q-List and Classification of Structures, Systems, and Components. This analysis contributes to the evaluation and modeling data used to support postclosure performance assessment. The soil depth conclusions of this analysis are not expected to affect the repository design or engineered features defined as important to safety per LS-PRO-0203. It is possible, however, that a future postclosure performance-assessment modeling report will use the conclusions of this analysis and the report could produce results that might affect a design or engineered feature. Such a modeling report would then address any potentially affected feature, structure, system, or component. 
Data Analysis for Infiltration Modeling: Technical Evaluation of Previous Soil Depth Estimation Methods and Development of Alternate Parameter Values

\section{INTENTIONALLY LEFT BLANK}




\section{USE OF SOFTWARE}

Digital comparisons were conducted using the baseline version of ARCINFO (ARCINFO V.7.2.1. STN: 10033-7.2.1-00 [DIRS 157019]) running on a Silicon Graphics, Inc. machine (CRWMS M\&O 2000 [DIRS 150920]). This geographic information system software is specifically designed for the intended use of graphically displaying the content of the files discussed herein and for comparing or compiling information for individual cells in the files. ARCINFO is the standard software used for geographic information system processing by the Technical Data Management System (TDMS) and it is appropriate for use in this analysis. This software is used within the range of validation.

Data compilation, sorting, and calculations presented in text, tables, and graphs in this analysis were conducted using standard mathematical and statistical functions in Microsoft ${ }^{\circledR} 2000$ Excel ${ }^{\circledR}$ running on a Dell ${ }^{\circ}$ personal computer using the Microsoft ${ }^{\circledR} 2000$ Windows ${ }^{\circledR}$ professional operating system. This software is exempt from the requirements of IT-PRO-0011, Software Management, Sections 1.4.2 and 1.4.6. Spreadsheets and workbooks with worksheets used for these processes are described in Appendix B, which also includes the electronic format on compact disc to meet the requirements for documentation and reproducibility per LP-SIII.9Q-BSC, Section 3 of Attachment 2. 
Data Analysis for Infiltration Modeling: Technical Evaluation of Previous Soil Depth Estimation Methods and Development of Alternate Parameter Values

\section{INTENTIONALLY LEFT BLANK}




\section{INPUTS}

\subsection{DIRECT INPUTS}

This analysis does not use direct inputs to evaluate the technical adequacy (Section 6.1) of existing soil depth data provided in SOILMAP6.INP (USGS 2000 [DIRS 175858], folder Soilmap6). The data used in Section 6.1, some of which are qualified and some unqualified, are used for comparison with the values in SOILMAP6.INP, and thereby determine whether the methods and values used for and in SOILMAP6.INP are corroborated.

Direct inputs used in the development of the alternate soil depth data summary (Section 6.2) are listed in Table 4-1. To define the ranges of soil depths found in each class discussed in Section 6.2.1, this analysis uses the following soil mapping sources, which have been catalogued in the TDMS by data tracking numbers (DTNs) and accession numbers (ACCs):

- GS940108315142.004 [DIRS 160344], ACCs: MOL.20021001.0238 and MOL.20021001.0239

- GS940108315142.005 [DIRS 160345], ACCs: MOL.20021203.0409 and MOL.20021203.0410

- GS940708315142.008 [DIRS 160346], ACCs: MOL.20021001.0260 and MOL.20021001.0261

- GS950408315142.004 [DIRS 160347], ACC: MOL.20020827.0355.

The analysis also uses a composite soils map (DTN: GS960408312212.005 [DIRS 146299], ACCs: JOL.19961118.0098 and MOL.1996 1118.0100), which was compiled from the sources just listed.

The composite soils map (DTN: GS960408312212.005 [DIRS 146299], ACCs: JOL.19961118.0098 and MOL.19961118.0100) is used to define soil depth classes (Section 6.2.1). The sources used are the current qualified and verified sources for soils mapping in the area covered in this analysis. The appropriateness of the composite soils map (DTN: GS960408312212.005 [DIRS 146299], ACCs: JOL.19961118.0098 and MOL.19961118.0100) has been evaluated separate from this analysis (BSC 2006 [DIRS 176335], Section 6).

Field surface measurements of soil depth are used as the primary source for soil depths in the shallow soil class (Table 4-1). The source DTN: GS011208312212.004 [DIRS 176317], Table S02086_001, provides better data on this depth range than do borehole data. Uncertainties associated with using borehole data in shallow soils are discussed in Section 6.2.

The direct inputs listed under the Borehole Soil Depth and Borehole Location data types are compiled in Table A-1. The data in Table A-1 include both qualified and unqualified inputs that are used in Sections 6-1 and 6-2. 
Data Analysis for Infiltration Modeling: Technical Evaluation of Previous Soil Depth Estimation Methods and Development of Alternate Parameter Values

Table 4-1. Direct Input Data

\begin{tabular}{|c|c|c|}
\hline Data Type & Input Description & Input Document Identification Number \\
\hline \multirow[t]{5}{*}{ Soil Mapping } & $\begin{array}{l}\text { Preliminary Surficial Materials Properties Map: } \\
\text { Soils of the Yucca Mountain Area, NV. } \\
\text { Submittal date: } 04 / 18 / 1996 \text {. }\end{array}$ & $\begin{array}{l}\text { DTN: GS960408312212.005 [DIRS 146299], } \\
\text { ACCs: JOL.19961118.0098 and } \\
\text { MOL.19961118.0100 }\end{array}$ \\
\hline & $\begin{array}{l}\text { Draft Surficial Deposits Map of the Northeast } \\
\text { Quarter of the Busted Butte } 7.5 \text {-Minute } \\
\text { Quadrangle. Submittal date: } 12 / 22 / 1993 \text {. } \\
\end{array}$ & $\begin{array}{l}\text { DTN: GS940108315142.004 [DIRS 160344], } \\
\text { ACCs: MOL.20021001.0238 and } \\
\text { MOL.20021001.0239 }\end{array}$ \\
\hline & $\begin{array}{l}\text { Draft Surficial Deposits Map of the Southern Half } \\
\text { of the Topopah Spring NW 7.5-Minute } \\
\text { Quadrangle. Submittal date: } 12 / 22 / 1993 .\end{array}$ & $\begin{array}{l}\text { DTN: GS940108315142.005 [DIRS 160345], } \\
\text { ACCs: MOL.20021203.0409 and } \\
\text { MOL.20021203.0410 }\end{array}$ \\
\hline & $\begin{array}{l}\text { Draft Surficial Deposits Map of the Northwest } \\
\text { Quarter of the Busted Butte 7.5-Minute } \\
\text { Quadrangle, Nye County, Nevada. } \\
\text { Submittal date: 07/27/1994. }\end{array}$ & $\begin{array}{l}\text { DTN: GS940708315142.008 [DIRS 160346], } \\
\text { ACCs: MOL.20021001.0260 and } \\
\text { MOL.20021001.0261 }\end{array}$ \\
\hline & $\begin{array}{l}\text { Draft Map of the Surficial Deposits of the } \\
\text { Southern Half of the Busted Butte } 7.5 \\
\text { Quadrangle, Nye County, Nevada. } \\
\text { Submittal date: } 04 / 03 / 1995 . \\
\end{array}$ & $\begin{array}{l}\text { DTN: GS950408315142.004 [DIRS 160347], } \\
\text { ACC: MOL.20020827.0355 }\end{array}$ \\
\hline $\begin{array}{l}\text { Field Surface } \\
\text { Measurement } \\
\text { of Soil Depth }\end{array}$ & $\begin{array}{l}\text { SN-USGS-SCl-113 V1: Empirical Calculation of } \\
\text { Soil Thickness Based on Field Measurements. } \\
\text { Submittal date: } 01 / 24 / 2002 \text {. } \\
\end{array}$ & $\begin{array}{l}\text { DTN: GS950408315142.004 [DIRS 160347], } \\
\text { ACC: MOL.20020827.0355 }\end{array}$ \\
\hline \multirow[t]{10}{*}{$\begin{array}{l}\text { Borehole Soil } \\
\text { Depth }\end{array}$} & $\begin{array}{l}\text { SN-USGS-SCI-113 V1: Empirical Calculation of } \\
\text { Soil Thickness Based on Field Measurements. } \\
\text { Submittal date: } 01 / 24 / 2002 \text {. }\end{array}$ & $\begin{array}{l}\text { DTN: GS011208312212.004 [DIRS 176317], } \\
\text { Table S02086_001 }\end{array}$ \\
\hline & $\begin{array}{l}\text { UE-25 RF\#13 Borehole Log of Soil and Other } \\
\text { Materials Above Bedrock. } \\
\text { Submittal date: 03/05/1999. }\end{array}$ & $\begin{array}{l}\text { DTN: MO9903BLOGSOIL.000 [DIRS 103790], } \\
\text { Table S99142_001 }\end{array}$ \\
\hline & $\begin{array}{l}\text { Graphical Lithologic Log of Borehole UE-25 } \\
\text { UZ\#16. Submittal date: } 11 / 22 / 1993 .\end{array}$ & $\begin{array}{l}\text { DTN: GS931208314211.047 [DIRS 110313] } \\
\text { ACC: NNA.19940411.0054 } \\
\text { (partially superseded DTN) }\end{array}$ \\
\hline & $\begin{array}{l}\text { Table of Contacts in Boreholes USW UZ-N57, } \\
\text { UZ-N58, UZ-N59, and UZ-N61. } \\
\text { Submittal date: 02/10/1994. }\end{array}$ & $\begin{array}{l}\text { DTN: GS940208314211.008 [DIRS 145581], } \\
\text { ACC: NNA.19940323.0344 }\end{array}$ \\
\hline & $\begin{array}{l}\text { Table of Contacts for the Tiva Canyon Tuff in } \\
\text { Borehole USW UZ-N38. } \\
\text { Submittal date: 03/10/1994. }\end{array}$ & $\begin{array}{l}\text { DTN: GS940308314211.011 [DIRS 145588], } \\
\text { ACC: MOL.19941101.0063 }\end{array}$ \\
\hline & $\begin{array}{l}\text { Graphical Lithologic Log of Borehole NRG-2 } \\
\text { (UE-25 NRG\#2), Yucca Mountain, Nevada. } \\
\text { Submittal date: 10/07/1993. }\end{array}$ & $\begin{array}{l}\text { DTN: GS931008314211.039 [DIRS 150009], } \\
\text { ACC: NNA.19940105.0085 }\end{array}$ \\
\hline & $\begin{array}{l}\text { Graphical Lithologic Log of Bore Hole USW } \\
\text { NRG-6. Submittal date: } 10 / 07 / 1993 \text {. }\end{array}$ & $\begin{array}{l}\text { DTN: GS931008314211.045 [DIRS 150010], } \\
\text { ACC: NNA.19940608.0248 }\end{array}$ \\
\hline & $\begin{array}{l}\text { Lithostratigraphic Contacts from } \\
\text { MO9811MWDGFM03.000 to be Qualified } \\
\text { Under the Data Qualification Plan, } \\
\text { TDP-NBS-GS-000001. } \\
\text { Submittal date: } 04 / 04 / 2000 \text {. }\end{array}$ & $\begin{array}{l}\text { DTN: MO0004QGFMPICK.000 } \\
\text { [DIRS 152554], Table S00214_001 }\end{array}$ \\
\hline & $\begin{array}{l}\text { Table of Contacts in Boreholes USW UZ-N33 and } \\
\text { USW UZ-N34. Submittal date: 02/10/1994. }\end{array}$ & $\begin{array}{l}\text { DTN: GS940208314211.006 [DIRS 152555], } \\
\text { ACC: NNA.19940414.0074 } \\
\text { (partially superseded DTN) }\end{array}$ \\
\hline & $\begin{array}{l}\text { Table of Contacts in Boreholes USW UZ-N31, } \\
\text { UZ-N32, UZ-N37. Submittal date: 02/10/1994. }\end{array}$ & $\begin{array}{l}\text { DTN: GS940208314211.005 [DIRS 152563], } \\
\text { ACC: NNA.19940323.0348 } \\
\text { (partially superseded DTN) }\end{array}$ \\
\hline
\end{tabular}


Data Analysis for Infiltration Modeling: Technical Evaluation of Previous Soil Depth Estimation Methods and Development of Alternate Parameter Values

Table 4-1. Direct Input Data (Continued)

\begin{tabular}{|c|c|c|}
\hline Data Type & Input Description & Input Document Identification Number \\
\hline \multirow[t]{13}{*}{$\begin{array}{l}\text { Borehole Soil } \\
\text { Depth } \\
\text { (Continued) }\end{array}$} & $\begin{array}{l}\text { Table of Contacts in Boreholes USW UZ-N53, } \\
\text { USW UZ-N54, and USW UZ-N55. } \\
\text { Submittal date: 02/01/1994. }\end{array}$ & $\begin{array}{l}\text { DTN: GS940208314211.003 [DIRS 152565], } \\
\text { ACC: NNA.19940323.0355 } \\
\text { (partially superseded DTN) }\end{array}$ \\
\hline & $\begin{array}{l}\text { Table of Contacts in Borehole USW UZ-N35. } \\
\text { Submittal date: } 02 / 10 / 1994 .\end{array}$ & $\begin{array}{l}\text { DTN: GS940208314211.007 [DIRS 155533], } \\
\text { ACC: NNA.19940414.0078 }\end{array}$ \\
\hline & $\begin{array}{l}\text { Table of Contacts for the Tiva Canyon Tuff in } \\
\text { Borehole UE-25 UZN\#63. } \\
\text { Submittal date: } 03 / 28 / 1994 .\end{array}$ & $\begin{array}{l}\text { DTN: GS940308314211.017 [DIRS 155534], } \\
\text { ACC: MOL.19941101.0064 }\end{array}$ \\
\hline & $\begin{array}{l}\text { Generalized Lithologic Log for Test Well } \\
\text { USW H-1. Submittal date: } 06 / 27 / 2001 .\end{array}$ & $\begin{array}{l}\text { DTN: MO0106STRATHFM.028 } \\
\text { [DIRS 155589], ACC: NNA.19870519.0103 }\end{array}$ \\
\hline & $\begin{array}{l}\text { Lithologic Log for Drill-Hole UE-25 P\#1. Submittal } \\
\text { date: 06/27/2001. }\end{array}$ & $\begin{array}{l}\text { DTN: MO0106STRATHFM.029 } \\
\text { [DIRS 155590], ACC: HQS.19880517.2633 }\end{array}$ \\
\hline & $\begin{array}{l}\text { Lithologic Log of Drill-Hole USW G-1. } \\
\text { Submittal date: } 06 / 27 / 2001 .\end{array}$ & $\begin{array}{l}\text { DTN: MO0106STRATHFM.030 } \\
\text { [DIRS 155591], ACC: NNA.19870406.0222 }\end{array}$ \\
\hline & $\begin{array}{l}\text { Litologic Description of Exploratory Drill Hole } \\
\text { USW G-2. Submittal date: 06/27/2001. }\end{array}$ & $\begin{array}{l}\text { DTN: MO0106STRATHFM.031 } \\
\text { [DIRS 155592], ACC: NNA.19870506.0143 }\end{array}$ \\
\hline & $\begin{array}{l}\text { Geotechnical Borehole Logs for the Waste } \\
\text { Handling Building, Yucca Mountain Project, } \\
\text { Nevada Test Site, Nevada, Version 7/16/03. } \\
\text { Submittal date: } 07 / 23 / 2003 \text {. }\end{array}$ & $\begin{array}{l}\text { DTN: GS030783114233.001 [DIRS 164561], } \\
\text { Tables S03299_001 through S03299_016 }\end{array}$ \\
\hline & $\begin{array}{l}\text { Yucca Mountain Site Characterization Project } \\
\text { Geology and Rock Structure Log for Drillhole } \\
\text { USW NRG-7/7A, Rev. } 1 \text {. } \\
\text { Submittal date: 02/06/1995. }\end{array}$ & $\begin{array}{l}\text { DTN: SNF29041993002.042 [DIRS 166437], } \\
\text { Table S97096_001 }\end{array}$ \\
\hline & $\begin{array}{l}\text { Sample Management Facility Geologic Logs for } \\
\text { Nye County Early Warning Drilling Program } \\
\text { (EWDP) Phase III. Submittal date: } 10 / 08 / 2002 \text {. }\end{array}$ & $\begin{array}{l}\text { DTN: MO0210SMFGLOG3.000 } \\
\text { [DIRS 175917], Table S02293_001 }\end{array}$ \\
\hline & $\begin{array}{l}\text { Yucca Mountain Site Characterization Project } \\
\text { Geology and Rock Structure Log for Drillhole } \\
\text { UE25 NRG-2A, Rev. } 1 . \\
\text { Submittal date: 02/06/1995. }\end{array}$ & $\begin{array}{l}\text { DTN: SNF29041993002.036 [DIRS 175928], } \\
\text { ACC: MOL.19950327.0071 }\end{array}$ \\
\hline & $\begin{array}{l}\text { Sample Management \& Drilling Department } \\
\text { Records Package for Borehole Records (Geologic } \\
\text { Logs) from the "Geotechnical Field Investigation } \\
\text { for the Waste Handling Building," } \\
\text { FWP-SB-00-003. Submittal date: 01/10/2001. }\end{array}$ & $\begin{array}{l}\text { DTN: MO0101SEPBGLOG.000 } \\
\text { [DIRS 157481], ACCs: MOL.20010125.0206, } \\
\text { MOL.20010125.0212, MOL.20010125.0194, } \\
\text { MOL.20010125.0188, MOL.20010125.0182, } \\
\text { MOL.20010125.0221, MOL.20010125.0200, } \\
\text { MOL.20010125.0224, MOL.20010125.0191 } \\
\end{array}$ \\
\hline & $\begin{array}{l}\text { Graphical Lithologic Log of Borehole NRG-2B } \\
\text { (UE-25 NRG\#2B), Yucca Mountain, Nevada. } \\
\text { Submittal date: 10/07/1993. }\end{array}$ & $\begin{array}{l}\text { DTN: GS931108314211.041 [DIRS 150011], } \\
\text { ACC: NNA.19940217.0156 } \\
\text { (partially superseded DTN) }\end{array}$ \\
\hline \multirow[t]{5}{*}{$\begin{array}{l}\text { Borehole } \\
\text { Location }\end{array}$} & $\begin{array}{l}\text { As-Built Location of UE-25 RF\#13 Borehole. } \\
\text { Submittal date: } 02 / 23 / 1999 .\end{array}$ & $\begin{array}{l}\text { DTN: MO9902ASBUBOLC.000 } \\
\text { [DIRS 103793], } \\
\text { /pub3/gis/baseline/test/mo9902asbubolc.zip }\end{array}$ \\
\hline & $\begin{array}{l}\text { Yucca Mountain Project (YMP) Borehole } \\
\text { Locations. Submittal date: } 06 / 23 / 1999 \text {. }\end{array}$ & $\begin{array}{l}\text { DTN: MO9906GPS98410.000 [DIRS 109059], } \\
\text { /pub3/gis/baseline/test/g98410.zip }\end{array}$ \\
\hline & $\begin{array}{l}\text { Generalized Lithologic Log for Test Well } \\
\text { USW H-1. Submittal date: } 06 / 27 / 2001 \text {. }\end{array}$ & $\begin{array}{l}\text { DTN: MO0106STRATHFM.028 } \\
\text { [DIRS 155589], ACC: NNA.19870519.0103 }\end{array}$ \\
\hline & $\begin{array}{l}\text { Lithologic Log for Drill-Hole UE-25 P\#1. Submittal } \\
\text { date: 06/27/2001. }\end{array}$ & $\begin{array}{l}\text { DTN: MO0106STRATHFM.029 } \\
\text { [DIRS 155590]; ACC: HQS.19880517.2633 }\end{array}$ \\
\hline & $\begin{array}{l}\text { Lithologic Log of Drill-Hole USW G-1. } \\
\text { Submittal date: } 06 / 27 / 2001 .\end{array}$ & $\begin{array}{l}\text { DTN: MO0106STRATHFM.030 } \\
\text { [DIRS 155591], ACC: NNA.19870406.0222 }\end{array}$ \\
\hline
\end{tabular}


Data Analysis for Infiltration Modeling: Technical Evaluation of Previous Soil Depth Estimation Methods and Development of Alternate Parameter Values

Table 4-1. Direct Input Data (Continued)

\begin{tabular}{|l|l|l|}
\hline \multicolumn{1}{|c|}{ Data Type } & \multicolumn{1}{|c|}{ Input Description } & \multicolumn{1}{|c|}{ Input Document Identification Number } \\
\hline $\begin{array}{l}\text { Borehole } \\
\text { Location } \\
\text { (Continued) }\end{array}$ & $\begin{array}{l}\text { Lithologic Description of Exploratory Drill Hole } \\
\text { USW G-2. Submittal date: 06/27/2001. }\end{array}$ & $\begin{array}{l}\text { DTN: MO0106STRATHFM.031 } \\
\text { [DIRS 155592], ACC: NNA.19870506.0143 }\end{array}$ \\
\cline { 2 - 3 } & $\begin{array}{l}\text { As-Built Borehole Locations Waste } \\
\text { Handling Building WHB Site Investigation. } \\
\text { Submittal date: 03/07/2001. }\end{array}$ & $\begin{array}{l}\text { DTN: MO0103GSC01031.000 [DIRS 157304], } \\
\text { /pub3/gis/baseline/test/mo0103gsc01031.zip }\end{array}$ \\
\cline { 2 - 3 } & $\begin{array}{l}\text { As-Built Survey of Nye County Early Warning } \\
\text { Drilling Program (EWDP) Phase III } \\
\text { Boreholes NC-EWDP-10S, NC-EWDP-18P, and } \\
\text { NC-EWDP-22S - Partial Phase III List. } \\
\text { Submittal date: 03/21/2002. }\end{array}$ & $\begin{array}{l}\text { DTN: MO0203GSC02034.000 [DIRS 168375], } \\
\text { /pub3/gis/baseline/test/ncewdp31.zip }\end{array}$ \\
\cline { 2 - 4 } & $\begin{array}{l}\text { As-Built Location for Surface Boreholes. } \\
\text { Submittal date: 06/20/2005. }\end{array}$ & $\begin{array}{l}\text { DTN: MO0506GSC05047.000 [DIRS 175919], } \\
\text { /pub3/gis/baseline/test/g05047.zip and } \\
\text { Cr-4902-sbh.x/s }\end{array}$ \\
\hline Modeled \\
Alluvial Depth & $\begin{array}{l}\text { Geologic Framework Model (GFM2000). } \\
\text { Submittal date: 12/18/2000. }\end{array}$ & $\begin{array}{l}\text { DTN: MO0012MWDGFM02.002 } \\
\text { [DIRS 153777], model file }\end{array}$ \\
\cline { 2 - 3 } & Geologic Framework Model (GFM2000). & BSC 2004 [DIRS 170029], Figure 6-10 \\
\hline
\end{tabular}

$\mathrm{ACC}=$ accession number; DTN = data tracking number.

Direct inputs for borehole soil depth and borehole location (Table 4-1) used in this evaluation are listed in Section 6.2.1, pages 6-34 and 6-38 to 6-41. Data extracted from the direct-use (qualified) and indirect-use (unqualified) sources for borehole alluvial thickness (soil depth) and borehole location are listed in Table A-1. Qualified data that are directly used are listed in Table 4-1 and are separated from unqualified data in the complete listing of all borehole data in Table A-1. Data from the qualified sources are used to estimate the distribution of soil depths and are appropriate for determining soil depth in thicker soil depth classes. These sources were identified through a search of available data on borehole lithology in the Records Information System (RIS) and in the TDMS. These sources represent available data that have been qualified and verified and are considered appropriate for the use described in Section 6.2.

Other sources of soil depth information, such as test pit data, were reviewed but were not directly used. Test pit data that are qualified and verified are located around the Waste Handling Building location and do not intersect bedrock. There are several boreholes in this area that do intersect bedrock and provide a better estimate of soil depth at this location. Other test pit and trench sources are not qualified or verified, and the test pits in this category do not intersect soil-bedrock contact. Some trenches that do intersect the soil-bedrock contact were not directly used because of their qualification status. Data sources for some boreholes are identified as superseded in the TDMS. These sources are documented in the following DTNs:

- GS931108314211.041 [DIRS 150011], ACC: NNA.19940217.0156 (partially superseded DTN)

- GS931208314211.047 [DIRS 110313], ACC: NNA.19940411.0054 (partially superseded DTN)

- GS940208314211.003 [DIRS 152565], ACC: NNA.19940323.0355 (partially superseded DTN) 
- GS940208314211.005 [DIRS 152563], ACC: NNA.19940323.0348 (partially superseded DTN)

- GS940208314211.006 [DIRS 152555], ACC: NNA.19940414.0074 (partially superseded DTN).

As noted in the description and comment fields on the Technical Data Information Form, the data in these DTNs are partially superseded and only a small section of the borehole log is superseded by another DTN. The superseding DTNs only provide revised data for the bedded tuff section between the Tiva Canyon and Topopah Springs tuffs and data on the rest of the section penetrated by the boreholes remains in the partially superseded DTNs. From these sources, only the data that were not superseded were used in this analysis. Therefore, the data used from these sources are not superseded, are qualified, and are appropriate for use in this analysis.

Sources for modeled alluvial depth (Table 4-1) were used in this analysis (Section 6.2.1) as sources for depth information on the deeper alluvial section; these sources are from the current version of the geologic framework model (BSC 2004 [DIRS 170029]) and in its output product DTN: MO0012MWDGFM02.002 [DIRS 153777], both hereafter referred to as the GFM. This model represents a synthesis of available geologic information on the site area and is appropriate for this purpose.

Equations that calculate standard statistical test values and tables of standard values (Gilbert 1987 [DIRS 163705]) were used to interpret the test results. The standard textbook used is an appropriate source for this type of information and is the type of source that scientists and engineers would use in their standard work practices to retrieve information of this type. This textbook and the methods taken from this textbook that are used in this analysis have also been recognized and recommended as sources for use in regulatory actions by the U.S. Environmental Protection Agency (EPA 2002 [DIRS 176873], p. 8-11).

\subsection{CRITERIA}

The technical work plan (BSC 2006 [DIRS 176107], Section 3) identifies the regulatory criteria applicable to infiltration modeling. A subset of these criteria is applicable to data evaluation and summarization for use in infiltration modeling. The activities described in this analysis are subject to regulatory review per the acceptance criteria in Yucca Mountain Review Plan, Final Report, NUREG-1804 (NRC 2003 [DIRS 163274], Section 2.2.1.3.5.3); acceptance criteria 2 and 3, related to infiltration data, are as follows:

- Acceptance Criterion 2: Data are sufficient for model justification:

(1) Climatological and hydrological values used in the license application (e.g., time of onset of climate change, mean annual temperature, mean annual precipitation, mean annual net infiltration, etc.) are adequately justified. Adequate descriptions of how the data were used, interpreted, and appropriately synthesized into the parameters are provided. 
(3) The effects of fracture properties, fracture distributions, matrix properties, heterogeneities, time-varying boundary conditions, evapotranspiration, depth of soil cover, and surface-water runoff and runon are considered, such that net infiltration is not underestimated.

(4) Sensitivity or uncertainty analyses are performed to assess data sufficiency and determine the possible need for additional data.

- Acceptance Criterion 3: Data uncertainty is characterized and propagated through the model abstraction:

(1) Models use parameter values, assumed ranges, probability distributions, and bounding assumptions that are technically defensible, reasonably account for uncertainties and variabilities, and do not result in an under-representation of the risk estimate.

(2) The technical bases for the parameter values used in this abstraction are provided.

(3) Possible statistical correlations are established between parameters in this abstraction. An adequate technical basis or bounding argument is provided for neglected correlations.

\subsection{CODES, STANDARDS, AND REGULATIONS}

Codes, standards, or regulations applicable to this work are defined in the technical work plan (BSC 2006 [DIRS 176107], Section 3). The acceptance criteria in Section 4.2 are primarily derived from 10 CFR 63.114 (a) and (b) [DIRS 176544]. No other codes, standards, or regulations have been identified as being applicable to this analysis. 
Data Analysis for Infiltration Modeling: Technical Evaluation of Previous Soil Depth Estimation Methods and Development of Alternate Parameter Values

\section{ASSUMPTIONS}

No assumptions were used in this analysis that do not have direct confirming data or evidence. Other assumptions that are confirmed or supported by direct-use or corroborating evidence are discussed in Section 6. 
Data Analysis for Infiltration Modeling: Technical Evaluation of Previous Soil Depth Estimation Methods and Development of Alternate Parameter Values

INTENTIONALLY LEFT BLANK 


\section{SCIENTIFIC ANALYSIS DISCUSSION}

SOILMAP6.INP (USGS 2000 [DIRS 175858], folder Soilmap6) is referred to extensively throughout this analysis. SOILMAP6.INP is an intermediate product output file that was generated using the SOILMAP6 V1.0 routine within the infiltration model report (BSC 2004 [DIRS 170007], Appendix G). Afterwards, SOILMAP6.INP (USGS 2000 [DIRS 175858], folder Soilmap6) was used as an input file to the infiltration model software INFIL (INFIL V.2.0. STN: 10307-2.0-00 [DIRS 139422]), which is documented in the infiltration model report (BSC 2004 [DIRS 170007]). SOILMAP6.INP (USGS 2000 [DIRS 175858], folder Soilmap6) was found in the records system without an assigned DTN.

DTN: MO0512SPASURFD.000 [DIRS 175870] was created to contain SOILMAP6.INP and to provide a traceable link to the file (Section 7). SOILMAP6.INP (USGS 2000 [DIRS 175858], folder Soilmap6) is an input file that consists of 49 columns and 253,597 rows for the infiltration model. Each row corresponds to a $30 \times 30 \mathrm{~m}$ cell in the infiltration model area. The columns provide information on cell location, soil type, soil depth, soil depth class, slope, aspect, elevation, geology, and blocking ridge angles.

The term "soil depth" is used herein to denote the thickness of all surficial deposits that overlie the bedrock at Yucca Mountain. Therefore, "soil" may range in composition from talus accumulations of cobbles and boulders to fine-grained eolian deposits.

Native Excel ${ }^{\circledR}$ spreadsheets, and workbooks with worksheets, of calculations used in this evaluation (Sections 6.1 and 6.2) are provided in Appendix B.

\subsection{EVALUATION}

\subsubsection{Background Information and Evaluation of SOILMAP6.INP}

The construction of SOILMAP6.INP (USGS 2000 [DIRS 175858], folder Soilmap6) is discussed in the infiltration model report (BSC 2004 [DIRS 170007]); SOILMAP6.INP was not an output or reference of the report. SOILMAP6.INP (USGS 2000 [DIRS 175858], folder Soilmap6) is based on a set of qualified and verified surficial deposits maps, generated by Lundstrom et al., documented in DTNs:

- GS940108315142.004 [DIRS 160344], ACC: MOL.20021001.0239

- GS940108315142.005 [DIRS 160345], ACC: MOL.20021203.0410

- GS940708315142.008 [DIRS 160346], ACC: MOL.20021001.0260

- GS950408315142.004 [DIRS 160347], ACC: MOL.20020827.0355.

These maps were later consolidated and modified using a different soil classification system developed by the U.S. Department of Agriculture (USDA 1994 [DIRS 175859]). The resulting map encompasses the infiltration modeling area (DTN: GS960408312212.005 [DIRS 146299], ACC: JOL.19961118.0098). It was Guertal and Davies who, to develop a new map, used soil descriptions and physical properties measurements from test pits and trenches in the Yucca Mountain area, along with the earlier mapping by Lundstrom et al. as listed in the previous paragraph. Creation of the new map consolidated the areas encompassed by the other maps onto 
one single map (DTN: GS960408312212.005 [DIRS 146299], ACC: JOL.19961118.0098); the source tree for this map lists twenty-five DTNs. This DTN and its sources are qualified and verified in accordance with procedures that have subsequently been superseded by LP-3.15Q-BSC.

The new map by Guertal and Davies (DTN: GS960408312212.005 [DIRS 146299], ACC: JOL.19961118.0098) and associated data (DTN: GS960508312212.007 [DIRS 124829], ACCs: MOL.20020930.0554 and MOL.20020930.0552), which give preliminary depth to bedrock, were used in the development of the infiltration model (BSC 2004 [DIRS 170007]) to estimate the thickness of surficial deposits by assigning soil depth codes. The preliminary depth to bedrock codes were further modified to remove unrealistically sharp contrasts between adjacent cells, remove inconsistencies between soil depth and bedrock classification, and assign discrete soil thicknesses. The assignment was then further modified by software routine GEOMOD4 V1.0 (BSC 2004 [DIRS 170007], Appendix F) to ensure that any contacts between thin colluvial deposits and deep alluvial deposits were separated by cells with an intermediate surficial deposit thickness. The same routine also ensured that any cells assigned a bedrock classification in the bedrock properties input file GEOMAP7.INP (BSC 2004 [DIRS 170007], Appendix F), which indicates a thin colluvial cover and deep alluvial classification based on soils mapping, received an intermediate depth value to reconcile the two classifications (BSC 2004 [DIRS 170007], Appendix F). Finally, a discrete thickness, based on the soil class and topographic slope angle for each $30 \times 30 \mathrm{~m}$ cell in the model grid, was calculated using the software routine SOILMAP6 V1.0 (BSC 2004 [DIRS 170007], Appendix G).

The calculation resulted in two values related to the soil depth in SOILMAP6.INP (USGS 2000 [DIRS 175858], folder Soilmap6) for each of the approximately 250,000 cells that compose the model area. The first soil depth value in the file shows one of six soil depth code numbers, as listed in Table 6-1, assigned to a cell. Soil Depth Codes 3, 5, and 6 represent the same depth range; the depth values, however, were calculated using different methods. The second soil depth value in the file provides an estimated thickness of the surficial deposit based on the soil depth code and topographic slope.

Table 6-1. Explanation of Soil Depth Codes Used in SOILMAP6.INP

\begin{tabular}{|c|l|l|}
\hline $\begin{array}{c}\text { Soil Depth } \\
\text { Code }\end{array}$ & $\begin{array}{c}\text { Depth Range } \\
\text { Represented }(\mathbf{m})\end{array}$ & \multicolumn{1}{c|}{ Comments } \\
\hline 1 & 0.0 to 0.5 & Depth based on soil type and topography \\
\hline 2 & 0.5 to 3.0 & Depth based on soil type and topography \\
\hline 3 & 3.0 to 6.0 & Depth based on soil type and topography \\
\hline 4 & $>6.0$ & Depth based on soil type and topography \\
\hline 5 & 3.0 to 6.0 & $\begin{array}{l}\text { Depth based on GEOMOD4 routine } \\
\text { Adds intermediate depth between deep and shallow-soil cells }\end{array}$ \\
\hline 6 & 3.0 to 6.0 & $\begin{array}{l}\text { Depth based on GEOMOD4 routine } \\
\text { Adds intermediate depth when cell mapped as bedrock, but has a deep } \\
\text { alluvial soil }\end{array}$ \\
\hline
\end{tabular}

Source: BSC 2004 [DIRS 170007], Section 6.6.2 and Appendices B and G.

NOTE: Depth ranges and precision are as given in the source. 
SOILMAP6.INP (USGS 2000 [DIRS 175858], folder Soilmap6) consists of a compact disc containing one folder, GEOINPUT-1, which contains four secondary folders: Blockr7, Geomap7, Geomod4, and Soilmap6 (USGS 2000 [DIRS 175858]). SOILMAP6.INP is included in the secondary folder Soilmap6, along with several other files related to the development of SOILMAP6.INP. Although GEOINPUT-1 was intended to be part of an attachment to previous versions of the infiltration model (USGS 2000 [DIRS 123650]; USGS 2001 [DIRS 154674]; USGS 2001 [DIRS 160355]; USGS 2003 [DIRS 166518]), the compact disc containing GEOINPUT-1 was not included in versions located in the Controlled Documents Information System.

\subsubsection{Evaluation of SOILMAP6.INP}

SOILMAP6.INP (USGS 2000 [DIRS 175858], folder Soilmap6) contains a field that identifies the soil type from the original map (DTN: GS960408312212.005 [DIRS 146299], ACC: JOL.19961118.0098) for each cell in the model area, which is the starting point of this evaluation. Soil type is a descriptive classification based upon taxonomy, which is an orderly arrangement for distinguishing soils using observable characteristics (USDA 1994 [DIRS 175859]). Figure 6-1 presents, in map form, the content of the soil type assignments from SOILMAP6.INP (USGS 2000 [DIRS 175858], folder Soilmap6). The SOILMAP6.INP map (Figure 6-1) was compared to the original map (DTN: GS960408312212.005 [DIRS 146299], ACC: JOL.19961118.0098) by overlaying the two maps on a light table, and the SOILMAP6.INP file was found to be a good representation of the original map. In addition, the digital version of the soilmap (DTN: MO9903COV96274.000 [DIRS 176571], /pub3/gis/baseline/geol/soils.e00) was compared to SOILMAP6.INP using the ARCINFO IDENTITY command, and all 253,597 cells in SOILMAP6.INP (USGS 2000 [DIRS 175858], folder Soilmap6) were found to match the source file (Section B.1.1).

To generate a soil depth file for the entire model area, the soil types (Figure 6-1) were grouped into general soil depth classes (Table 6-2). Three main soil depth classes of soil, those being deep (Unit A), intermediate (Unit B), and shallow (Unit C) (Figure 6-2), were initially selected (DTN: GS960508312212.007 [DIRS 124829], ACCs: MOL.20020930.0554 and MOL.20020930.0552) to produce depth classes based solely on the soil mapping that correspond, respectively, to Soil Depth Codes 3, 2, and 1 (Table 6-1). The resulting soil depth file was then modified based on an estimate using a combination of surficial deposits mapping, topography, field observations, and soil-alluvial depth recorded at borehole sites to produce a final soil depth file. The final soil depth codes from the final file are listed in Table 6-1 and are shown in Figure 6-3. This final step, of combining the various sources of information, used the professional judgment of the author or authors of the infiltration model report (BSC 2004 [DIRS 170007]) who also created the map and soil-depth calculations. This professional judgment is unique to each individual and is not recorded in detail in the report or supporting records, and, thus, cannot be reproduced exactly, but the result has been evaluated against the combined soil map (Figure 6-2). 


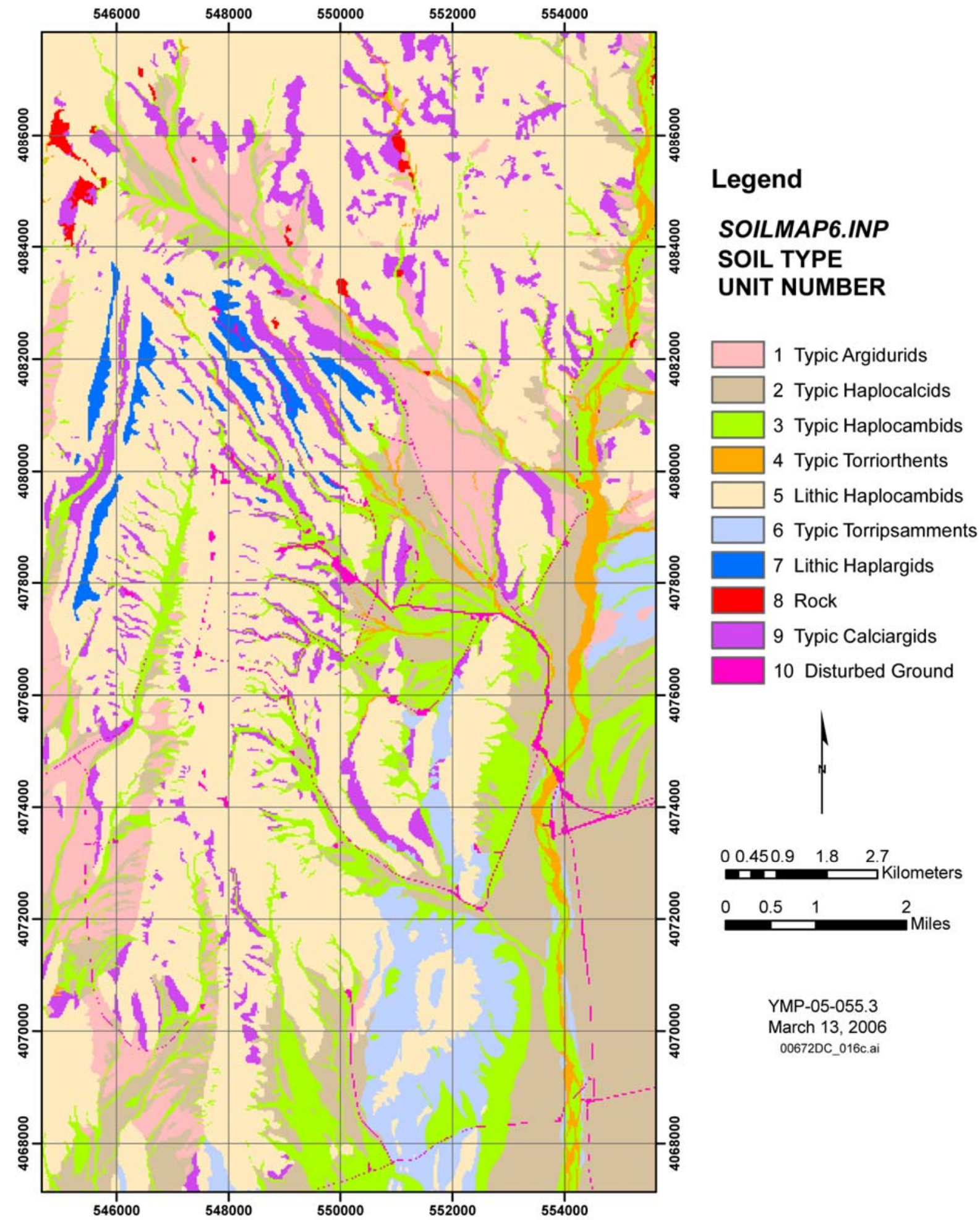

Sources: DTN: GS960408312212.005 [DIRS 146299], ACC: JOL.19961118.0098; USGS 2000 [DIRS 175858], folder Soilmap6.

NOTE: $\quad$ Map units are defined in Table 6-2.

Figure 6-1. Representation of the Digital Soil Map Using SOILMAP6.INP 
Data Analysis for Infiltration Modeling: Technical Evaluation of Previous Soil Depth Estimation Methods and Development of Alternate Parameter Values

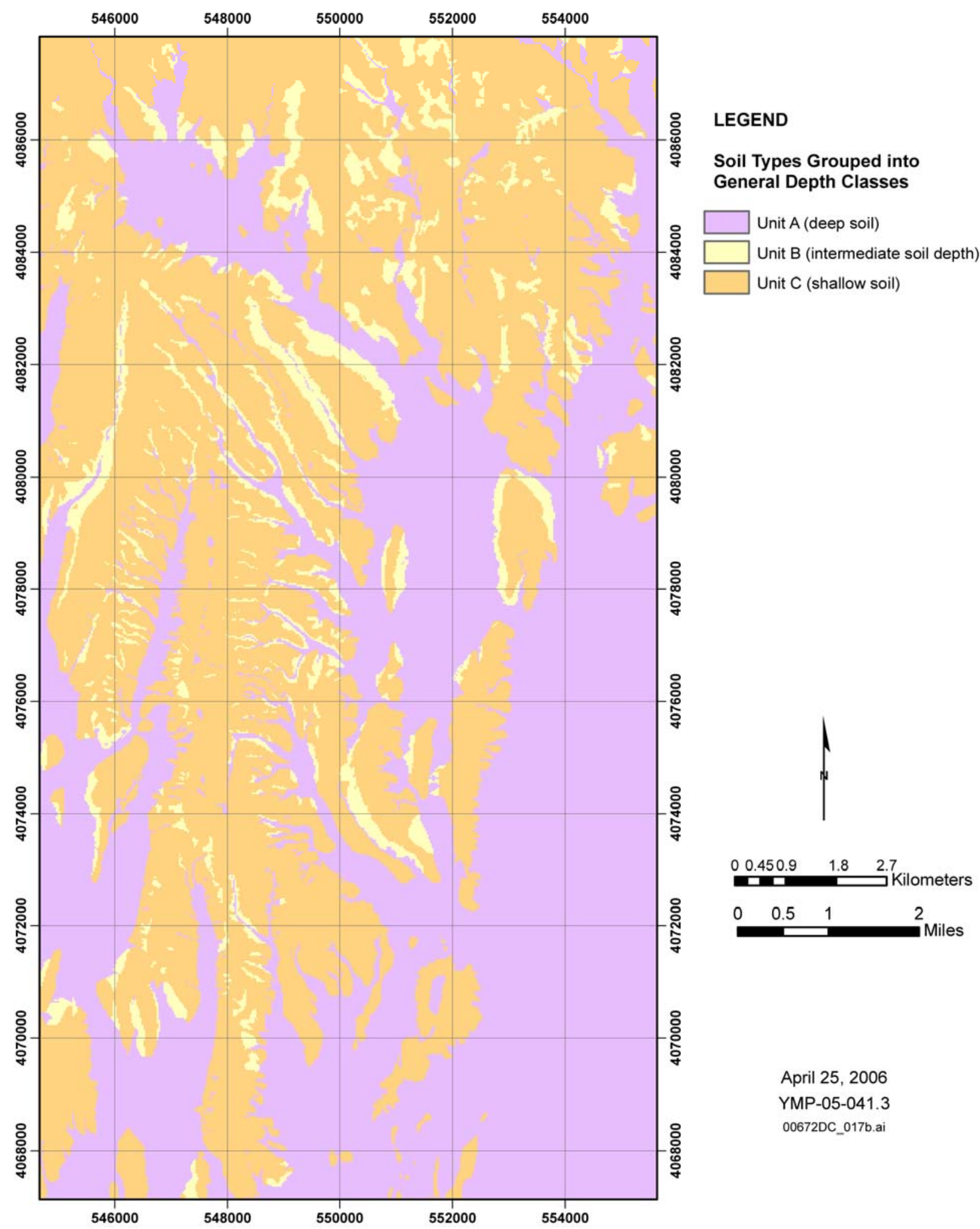

Sources: Figure 6-1; Table 6-2; USGS 2000 [DIRS 175858], folder Soilmap6.

Figure 6-2. Surficial Deposit Units Grouped into Depth Classes 
Data Analysis for Infiltration Modeling: Technical Evaluation of Previous Soil Depth Estimation Methods and Development of Alternate Parameter Values

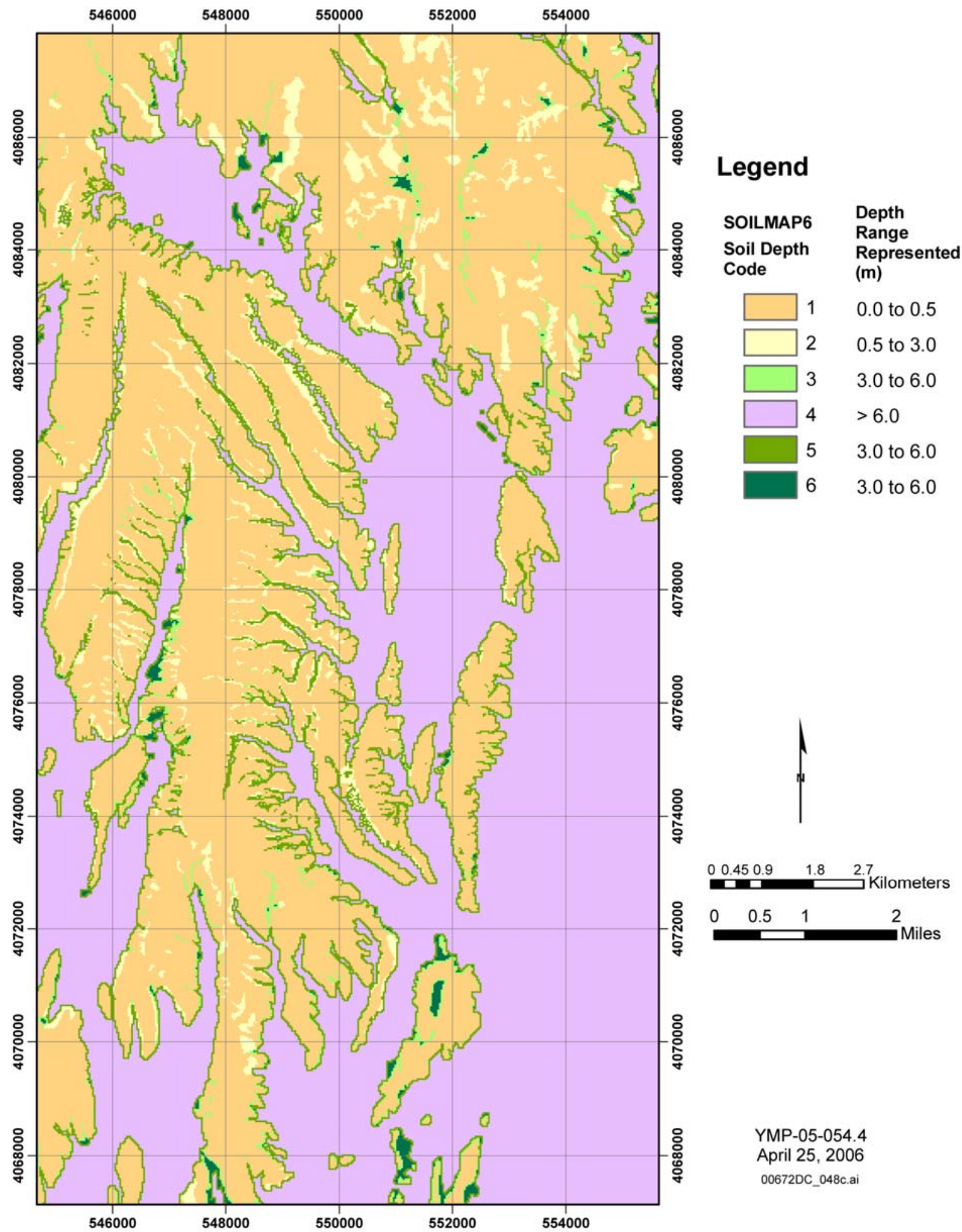

Source: USGS 2000 [DIRS 175858], folder Soilmap6.

NOTE: Map units are defined in Table 6-1.

Figure 6-3. Final Soil Depth Codes in SOILMAP6.INP 
Data Analysis for Infiltration Modeling: Technical Evaluation of Previous Soil Depth Estimation Methods and Development of Alternate Parameter Values

Table 6-2. Classification Scheme Used in the Digital Soil Map for Surficial Deposits Mapping

\begin{tabular}{|c|c|c|c|}
\hline $\begin{array}{c}\text { Soil Type } \\
\text { Unit Number }^{\mathrm{a}}\end{array}$ & Soil Classification $^{a}$ & $\begin{array}{l}\text { Corresponding General } \\
\text { Soil Depth Classes }\end{array}$ & $\begin{array}{l}\text { Lundstrom Soil } \\
\text { Classification }^{c}\end{array}$ \\
\hline 1 & Typic Argidurids & Deep Soil-Alluvium & $0,1,1-3,2$, Tgp \\
\hline 2 & Typic Haplocalcids & Deep Soil-Alluvium & $\begin{array}{l}3,3 f, 3-4,4,4 f, 4 s, 4 / 1,4 s-5 s \\
3-5\end{array}$ \\
\hline 3 & Typic Haplocambids & Deep Soil-Alluvium & $\begin{array}{l}5,5 f, 5 s, 5 / 1,5-6,5 f-6 f, 6,6 f \\
5-7\end{array}$ \\
\hline 4 & Typic Torriorthents & Deep Soil-Alluvium & $7,7 f, 6-7,6 f-7 f$ \\
\hline 5 & Lithic Haplocambids & Shallow Soil-Colluvium & $\mathrm{cu}, \mathrm{cs}$ \\
\hline 6 & Typic Torripsamments & Deep Soil-Alluvium & e, eo, ey, $1 /$ eo, $3 /$ eo, $1 / e, 3 / e$ \\
\hline 7 & Lithic Haplargids & Shallow Soil-Colluvium & $\mathrm{rc}$ \\
\hline 8 & Rock & Exposed Bedrock-No Surficial Deposits & $\mathrm{r}$ \\
\hline 9 & Typic Calciargids & Intermediate Soil & $\mathrm{cf}, \mathrm{cf} / \mathrm{e}$ \\
\hline 10 & Disturbed Ground & Roads and Drill Pads & d \\
\hline
\end{tabular}

a DTN: GS960408312212.005 [DIRS 146299], ACC: JOL.19961118.0098

b Grouping of soil classifications into depth classes shown in DTN: GS960508312212.007 [DIRS 124829], ACC: MOL.20020930.0554

c DTNs: GS940108315142.004 [DIRS 160344], ACC: MOL.20021001.0238 GS940108315142.005 [DIRS 160345], ACC: MOL.20021203.0409 GS940708315142.008 [DIRS 160346], ACC: MOL.20021001.0261 GS950408315142.004 [DIRS 160347], ACC: MOL.20020827.0355

The final soil depth map (Figure 6-3) reasonably correlates to the consolidated map (Figure 6-2), indicating that the soil depth classes are a good, general representation of depth based on soil mapping. Modifications introduced by the GEOMOD4 routine (BSC 2004 [DIRS 170007], Appendix F) were then incorporated into the soil depth code map. The extent of Soil Depth Codes 5 and 6 resulting from the use of this routine is relatively limited (Figure 6-3), so the effect of adding soil depth codes 5 and 6 will be small with respect to the total infiltration for the entire model area. The soil depths in meters from SOILMAP6.INP (USGS 2000 [DIRS 175858], folder Soilmap6) were then calculated using a soil depth model that relies on soil depth code and topographic slope. The principal assumption in this calculation is that soil-colluvial depth varies with topography; for example, flatter ridge crests and areas at the base of steep slopes tend to have thicker accumulations of surficial deposits than the deposits found on steep hillside slopes.

The following formulae were used to calculate soil depth in meters (D) (BSC 2004 [DIRS 170007], Section 6.7.1):

Soil Depth Code 1

$\mathrm{D}=0.03 * \mathrm{~S}+0.1$ for slope angles $(\mathrm{S})$ less than or equal to 10 degrees

$\mathrm{D}=0.013 *(10-\mathrm{S})+0.4$ for slope angles between 10 and 40 degrees

$\mathrm{D}=0.01$ for slope angles equal to or greater than 40 degrees.

Soil Depth Code 2

$\mathrm{D}=2-(0.05 * \mathrm{~S})$ for slope angles less than 32 degrees

$\mathrm{D}=0.4$ for slope angles equal to or greater than 32 degrees.

Soil Depth Code 3

$\mathrm{D}=6-(0.16 * \mathrm{~S})$ for slope angles less than 25 degrees

$\mathrm{D}=2.0$ for slope angles equal to or greater than 25 degrees. 
Soil depth for Soil Depth Code 4 was uniformly set at $6 \mathrm{~m}$ for all cells, even though most actual depths are greater than this value. This was established because the infiltration model assumes that soil thicknesses exceeding $6 \mathrm{~m}$ eliminate the infiltration of water to the soil-bedrock contact, except in some stream channels (BSC 2004 [DIRS 170007], Section 6.1.2), and because the root zone depth is considered to not exceed $6 \mathrm{~m}$, so depth calculations are not required for this code.

A check conducted on the first 65,536 rows in SOILMAP6.INP (USGS 2000 [DIRS 175858], folder Soilmap6), using Excel ${ }^{\circledR}$ (Section B.1.2), found that the depth values in the file could be reproduced using the equations in the infiltration model report (BSC 2004 [DIRS 170007], Section G4) and by using the soil depth codes assigned in the file. The only problem noted was a typographical error in the equation for depth class 3 (BSC 2004 [DIRS 170007], Appendix B), which indicates that the first equation for this class should be used for slope angles less than or equal to 25 degrees. Contrary to this range, the infiltration model report (BSC 2004 [DIRS 170007], Section 6.7.1) and SOILMAP6.INP (USGS 2000 [DIRS 175858], folder Soilmap6) indicate that the equation should be used for slope angles less than 25 degrees and that the constant value of 2.00 should be used for slope angles greater than or equal to 25 degrees.

The infiltration model report (BSC 2004 [DIRS 170007]) and its predecessor reports (USGS 2003 [DIRS 166518]; USGS 2001 [DIRS 160355]; USGS 2000 [DIRS 123650]) do not indicate how these depth relationships were derived, so the accuracy of these relationships cannot be verified directly. The equations appear to be linear fits between certain points in the range of slope angles. For example, for the Soil Depth Code 1 equations just given, the ranges are defined by 0 to 10 degrees, between 10 and 40 degrees, and 40 degrees and greater. It is unclear as to how these ranges were chosen or how the constants used in each equation were determined.

The depth equations also define different end points for the soil depth codes than are identified in the infiltration model report (BSC 2004 [DIRS 170007]). For example, the infiltration model report (BSC 2004 [DIRS 170007], Section 6.6.2) gives the depth range for Soil Depth Code 1 as 0 to $0.5 \mathrm{~m}$. The formulae discussed herein, however, define a depth range of 0.01 to $0.4 \mathrm{~m}$. Similarly, the depth range given in the infiltration model report (BSC 2004 [DIRS 170007]) for Soil Depth Code 2 is 0.5 to $3.0 \mathrm{~m}$, while the formulae define a range between 0.4 and $2.0 \mathrm{~m}$. Soil Depth Code 3 is also at variance with a range of 3.0 to $6.0 \mathrm{~m}$ (BSC 2004 [DIRS 170007]); the formulae define a range between 2.0 and $6.0 \mathrm{~m}$. This variance calls into question either the soil-depth-code definitions or the depth calculation formulae. Whichever rationale is used to calculate the depth value of cells, the result can be evaluated using other lines of evidence as discussed herein.

One set of data contains field measurements that provide soil depth and a corresponding slope value (DTN: GS011208312212.004 [DIRS 176317], Table S02086_001). The formulae can be evaluated by using the formulae to calculate depth from the measured slope value and then comparing the result to the measured depth value at that point. This can be done for two equations by assuming that the data, classed as having an underlying material of "Tpc" and "stone stripe" (DTN: GS011208312212.004 [DIRS 176317], Table S02086_001), correspond to Soil Depth Code 1 (DTN: MO0512SPASURFD.000 [DIRS 175870]). 
The results from calculating depths using the equation for slopes of less than or equal to 10 degrees (Table 6-3, Figure 6-5) show a moderate degree of correlation between the calculated and measured values (correlation coefficient; $r=0.71, r^{2}=0.50$ ). Depths calculated using the equation for slopes between 10 and 40 degrees show a poor degree of correlation between the calculated and measured values (correlation coefficient; $r=0.24, r^{2}=0.06$ ). Three outlying points may affect the comparison (Figure 6-4). A test with these points removed, however, still indicates a poor correlation (correlation coefficient; $r=0.50, \mathrm{r}^{2}=0.25$ ).

The scatter of points resulting from this comparison (Figures 6-4 and 6-5) indicates that, at the detailed level, the correlation of topographic slope to soil depth is subject to uncertainty. This measured soil depth and slope data are not discussed in the infiltration model report (BSC 2004 [DIRS 170007]), even though a principal infiltration investigator participated in the data collection effort and the purpose of the data collection effort is stated as being to develop an empirical relationship to calculate soil thickness for modeling recharge in the unsaturated zone at Yucca Mountain (Taylor 2001 [DIRS 176601], RIS p. 5).

The soil depth file in SOILMAP6.INP (USGS 2000 [DIRS 175858], folder Soilmap6) can also be evaluated on a more general level. Figure 6-6 shows the soil depth values from SOILMAP6.INP (USGS 2000 [DIRS 175858], folder Soilmap6) for a small part of the infiltration model area around The Prow at the north end of Yucca Mountain.

As expected from the equations used, the map mimics the topography in the area (Figures 6-6 and 6-7). Depth values on ridge crests are thin and grade to near zero on steep slopes surrounding the ridge crests. At the base of the steep slopes, the shallower slopes leading down to major drainages show increasing surficial deposit thickness. Thick alluvial deposits exist in the vicinities of major drainages, such as Windy Wash and Yucca Wash. This general pattern is reasonable and consistent with field observations (Sanchez 2006 [DIRS 176569], pp. 62 to 68).

While the general pattern is consistent and reasonable, the assignments to individual grid cells cannot be verified directly and may be more variable than the input file indicates. At a more detailed level, soil depth might be highly variable, even within a $30 \times 30 \mathrm{~m}$ grid cell. Soil depths can range from small patches of bare rock to $0.5 \mathrm{~m}$ or more within a cell (Sanchez 2006 [DIRS 176569], photographs; Taylor 2001 [DIRS 176601], photographs). The methods used to estimate soil depths in a grid cell in the infiltration model report (BSC 2004 [DIRS 170007]) cannot account for this local variability. Thus, users of these depth estimates should be aware of this scale-dependent uncertainty when using these depth estimates.

Another check was performed by comparing surficial deposit depth information in boreholes to the predictions of SOILMAP6.INP (USGS 2000 [DIRS 175858], folder Soilmap6). Table A-1 shows the boreholes, values, and data sources used for this comparison. The use of borehole data to verify surficial deposit depth is subject to some caveats that must be considered in making such a comparison. One consideration is the effect from drill pad construction on the measurement. In uneven terrain, the construction of a level pad for the drill rig may require significant cutting or filling, or both. If a borehole is shown as starting directly in bedrock, then this may indicate that surficial deposits were removed down to the bedrock as part of pad construction. This factor is apparent (Sanchez 2006 [DIRS 176569]) where the depth of surficial deposits, removed by pad construction, is indicated for some boreholes. 
Data Analysis for Infiltration Modeling: Technical Evaluation of Previous Soil Depth Estimation Methods and Development of Alternate Parameter Values

Table 6-3. Comparison of Measured Soil Thickness with Calculated Thickness

\begin{tabular}{|c|c|c|c|}
\hline $\begin{array}{c}\text { Slope Magnitude } \\
\text { (degrees) }^{\mathrm{a}}\end{array}$ & $\begin{array}{l}\text { Measured Soil } \\
\text { Thickness (m) }\end{array}$ & $\begin{array}{c}\text { Calculated Soil Thickness } \\
\text { using Slope }(m)^{b}\end{array}$ & $\begin{array}{c}\text { Difference Between Calculated } \\
\text { and Measured Value }\end{array}$ \\
\hline 1.5 & 0.10 & 0.145 & 0.045 \\
\hline 2.5 & 0.10 & 0.175 & 0.075 \\
\hline 2.5 & 0.20 & 0.175 & -0.025 \\
\hline 4.0 & 0.20 & 0.22 & 0.020 \\
\hline 5.0 & 0.20 & 0.25 & 0.050 \\
\hline 7.0 & 0.20 & 0.31 & 0.110 \\
\hline 10.0 & 0.75 & 0.4 & -0.350 \\
\hline 10.0 & 0.25 & 0.4 & 0.150 \\
\hline 11.5 & 0.25 & 0.381 & 0.131 \\
\hline 12.0 & 0.50 & 0.374 & -0.126 \\
\hline 14.0 & 0.40 & 0.348 & -0.052 \\
\hline 14.0 & 0.50 & 0.348 & -0.152 \\
\hline 15.0 & 3.00 & 0.335 & -2.665 \\
\hline 16.0 & 0.12 & 0.322 & 0.202 \\
\hline 16.0 & 0.45 & 0.322 & -0.128 \\
\hline 17.0 & 0.30 & 0.309 & 0.009 \\
\hline 20.0 & 0.50 & 0.270 & -0.230 \\
\hline 22.0 & 0.20 & 0.244 & 0.044 \\
\hline 22.0 & 1.00 & 0.244 & -0.756 \\
\hline 22.5 & 0.25 & 0.238 & -0.013 \\
\hline 24.0 & 0.30 & 0.218 & -0.082 \\
\hline 25.0 & 0.10 & 0.205 & 0.105 \\
\hline 25.0 & 3.00 & 0.205 & -2.795 \\
\hline 26.5 & 0.25 & 0.186 & -0.065 \\
\hline 27.0 & 0.20 & 0.179 & -0.021 \\
\hline 31.5 & 0.15 & 0.121 & -0.029 \\
\hline 31.5 & 0.20 & 0.121 & -0.080 \\
\hline 31.5 & 0.35 & 0.121 & -0.230 \\
\hline 32.0 & 0.05 & 0.114 & 0.064 \\
\hline 32.0 & 0.10 & 0.114 & 0.014 \\
\hline 32.0 & 0.40 & 0.114 & -0.286 \\
\hline 33.0 & 0.40 & 0.101 & -0.299 \\
\hline 34.0 & 0.17 & 0.088 & -0.082 \\
\hline 36.0 & 0.10 & 0.062 & -0.038 \\
\hline 43.0 & 0.40 & 0.010 & -0.390 \\
\hline
\end{tabular}

Source: Appendix B, Section B.1.3.

a Values from DTN: GS011208312212.004 [DIRS 176317], Table S02086_001, for Deposit Units “Tpc" and "stone st".

${ }^{b}$ Value calculated from slope value using the equations $D=0.03 \times S+0.1$ for $S \leq 10$; $D=0.013 \times(10-S)+0.4$ for $10<S<40$; and $D=0.01$ for $S>40$ degrees.

NOTE: Correlation Coefficient: $r=0.71$ for $S \leq 10$ and $r=0.24$ for $10<S<40$. 
Data Analysis for Infiltration Modeling: Technical Evaluation of Previous Soil Depth Estimation Methods and Development of Alternate Parameter Values

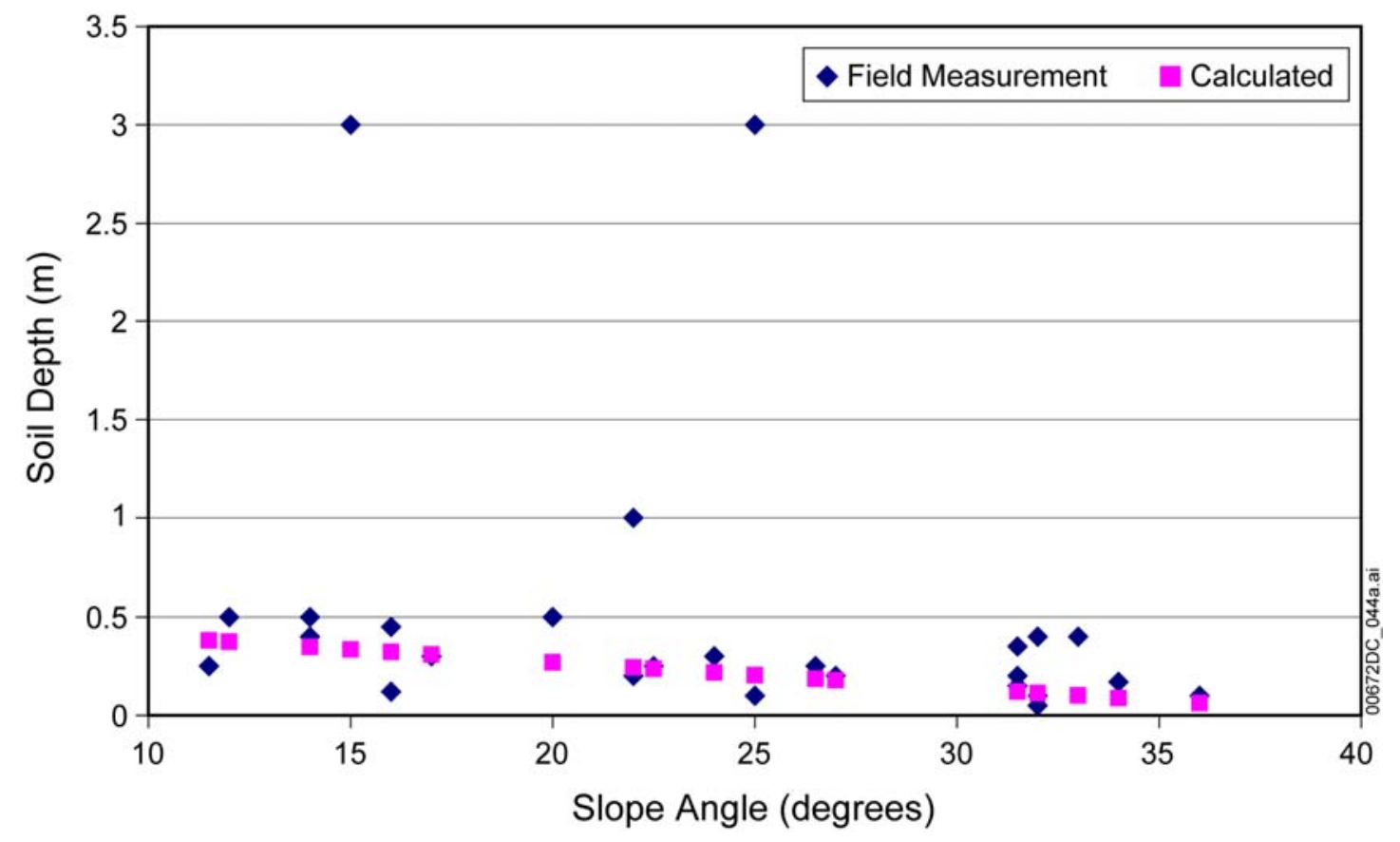

Source: Table 6-3; Appendix B, Section B.1.3 and Taylor soil depth.xls, worksheet 'Final calc'.

Figure 6-4. Comparison of Field Measurements to Depths Calculated Using Equation

$D=0.013 \times(10-S)+0.4$ for $10<S<40$ Degrees

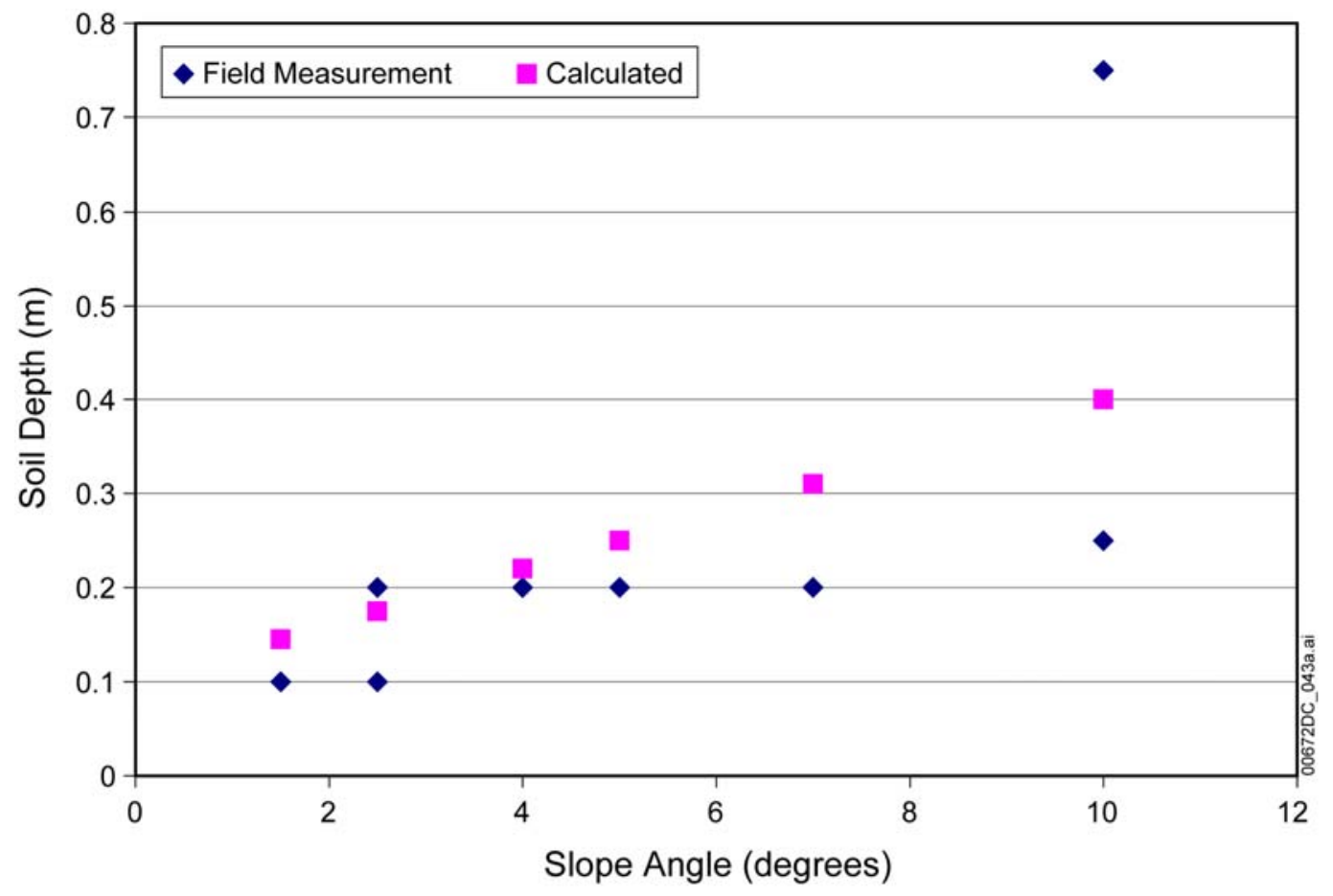

Source: Table 6-3; Appendix B, Section B.1.3 and Taylor soil depth.xls, worksheet 'Final calc'.

Figure 6-5. Comparison of Field Measurements to Depths Calculated Using Equation $D=0.03 \times S+0.1$ for $\mathrm{S} \leq 10$ Degrees 


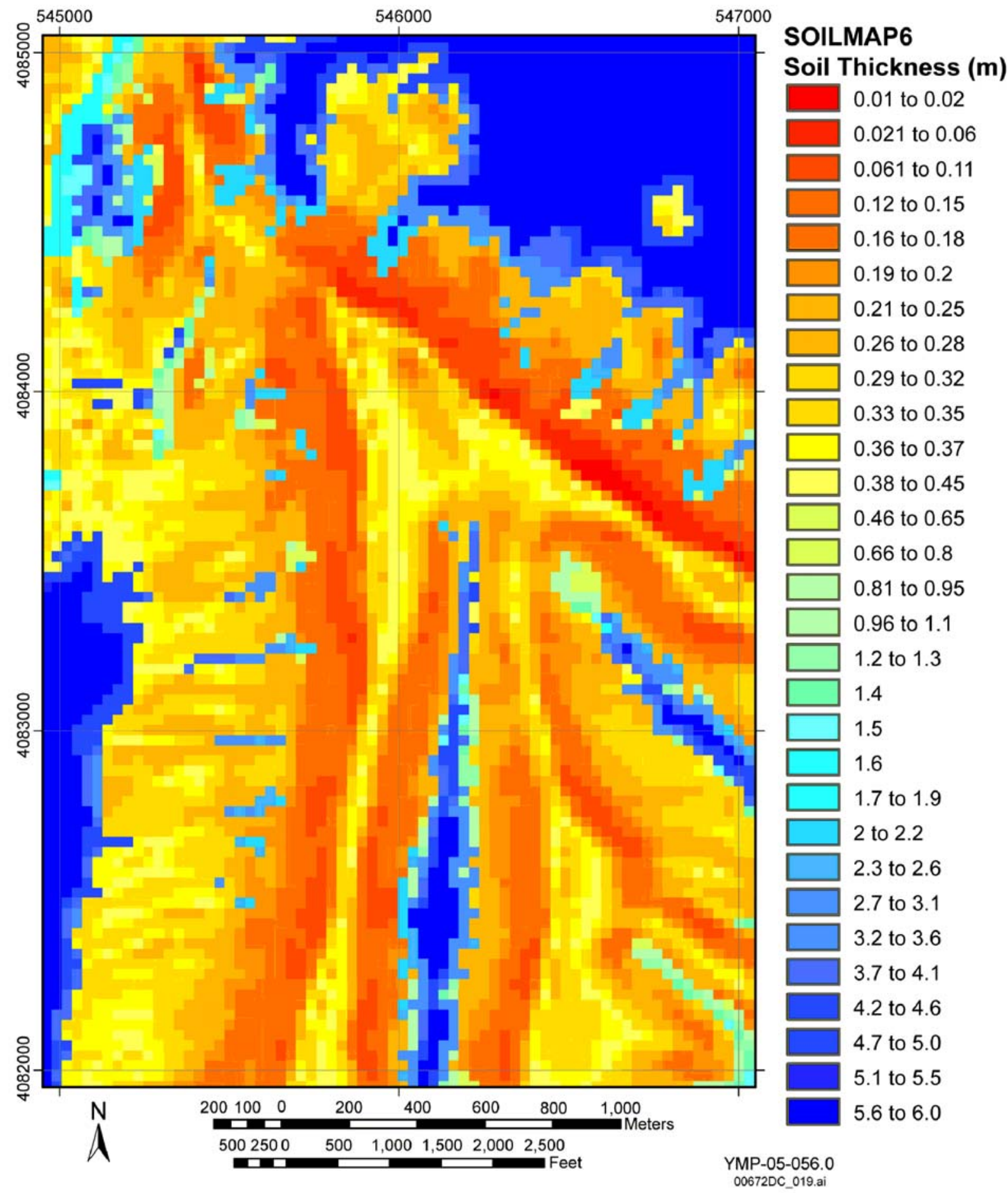

Source: USGS 2000 [DIRS 175858], folder Soilmap6.

Figure 6-6. Enlargement of the Area around the Prow Showing Soil Depth Values from SOILMAP6.INP 
Data Analysis for Infiltration Modeling: Technical Evaluation of Previous Soil Depth Estimation Methods and Development of Alternate Parameter Values

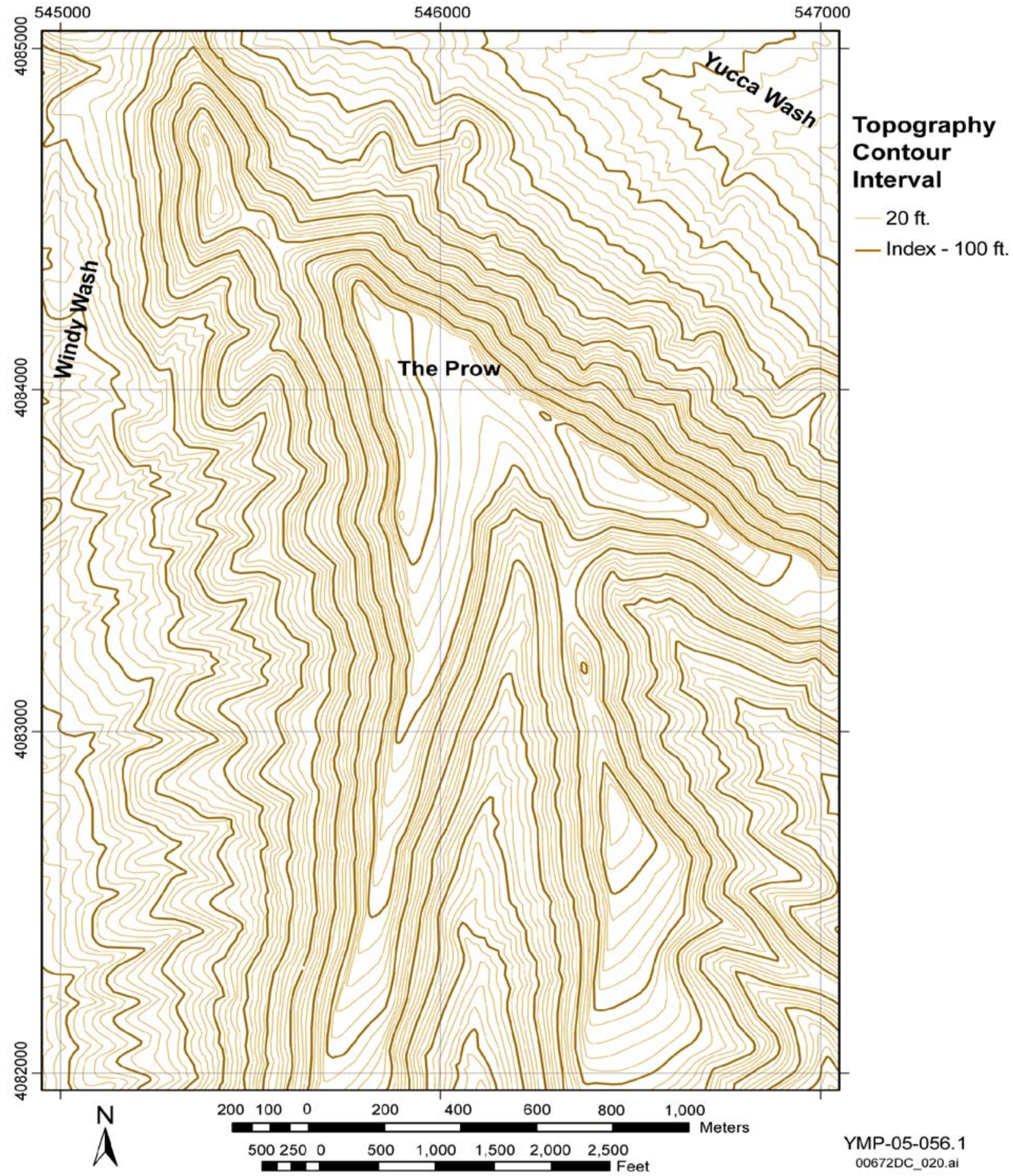

Source: DTN: MO9811COV98591.000 [DIRS 109060], /pub3/gis/baseline/topo/topo20s.e00.

Figure 6-7. Topographic Map for the Area Covered in Figure 6-6 
Examples of boreholes with a zero thickness (Table A-1) due to drill pad construction are:

- UE-25 NRG\#3: 0.3 to $0.5 \mathrm{~m}$ soil thickness around cut (Sanchez 2006 [DIRS 176569], pp. 62 and 63)

- UE-25 NRG\#5: 0.5 to $3 \mathrm{~m}$ soil thickness around cut (Sanchez 2006 [DIRS 176569], pp. 48 and 50)

- USW SD-9: 0.0 to $0.9 \mathrm{~m}$ soil thickness around cut (Sanchez 2006 [DIRS 176569], p. 66)

- USW WT-24: $0.2 \mathrm{~m}$ soil thickness around cut (Sanchez 2006 [DIRS 176569], p. 67)

- USW H-5: 0.3 to $0.5 \mathrm{~m}$ soil thickness around cut (Sanchez 2006 [DIRS 176569], pp. 59 and 67).

Conversely, some boreholes may penetrate significant thicknesses of artificial fill before entering naturally occurring deposits. When borehole logs differentiate between pad fill and natural alluvium, only the alluvial thickness is shown in Table A-1. It is uncertain that such distinctions were always made, because the primary interest in most borehole logging was determining bedrock geology and not the details of the alluvial section.

There are also a limited number of boreholes available with which to make a comparison. With the exception of the Waste Handling Building area in Midway Valley, boreholes are widely scattered. Some areas, such as the area north of Yucca Wash, have no borehole information. In addition, borehole logs give a value only at a specific point and, with a limited number of boreholes they provide minimal information on how the variability in depth of surficial deposits across the entire model area should be modeled. Therefore, boreholes provide a limited number of spot checks that can be used to corroborate an area-wide model.

The depth of surficial deposits in boreholes is compared graphically (Figure 6-8) to soil depth codes (Figure 6-3). Given the limitations of borehole measurements, as previously discussed, the correlation between the two sources is good. Nearly all of the boreholes match the applicable soil depth code generated from the surficial deposits mapping (Figure 6-9). On the ridge crests, there are only minor variances between the two shallowest soil depth codes. In other areas, variances occur near the contacts with different soil depth codes, are in areas of rapid depth change, and are usually only off by a single soil depth code.

Table 6-4 provides a comparison of the calculated depth for the cell in which the borehole occurs against the value obtained from the borehole. It also shows the reasonably good correlation between the two approaches for the shallowest Soil Depth Codes 1 and 2, being that the borehole data indicate that the cells with boreholes fall into either of these codes. The data, however, also indicate that the precision of the depth calculation is not high, with an average difference of about plus or minus $1.0 \mathrm{~m}$ for the depth calculation in the shallowest soil depth codes. Figure 6-10 illustrates the correlation between soil depth indicated by borehole data and the value for the corresponding cell predicted by SOILMAP6.INP (USGS 2000 [DIRS 175858], folder Soilmap6). 


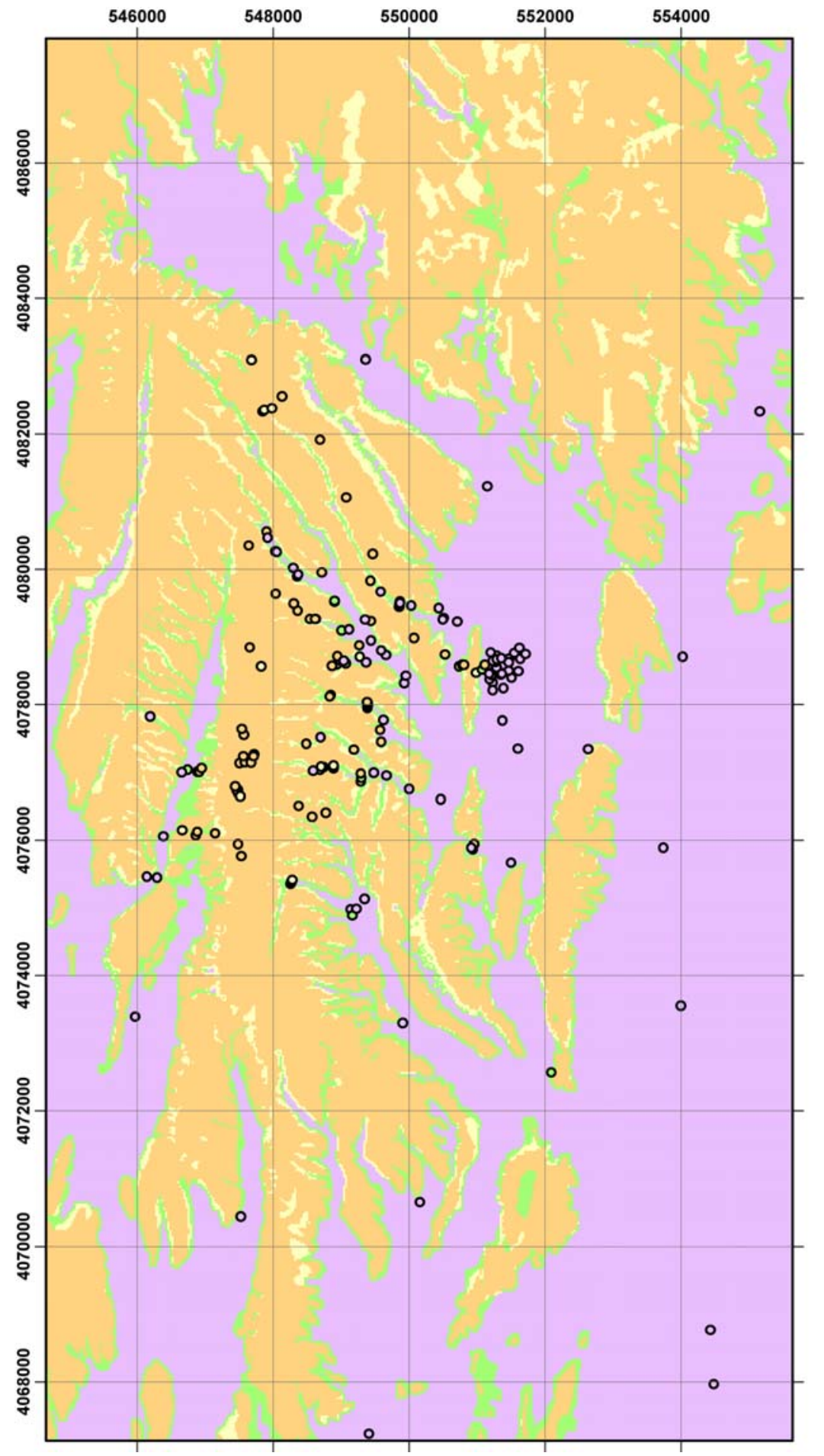

\section{Legend}

SOILMAP6.INP

Soil Depth (m)

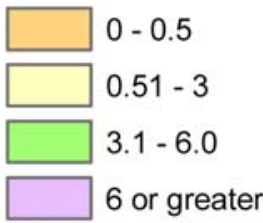

\section{Borehole Soil Depth (m)}

$\circ \quad 0-0.5$

$\circ \quad 0.51-3$

- $\quad 3.01-5.99$

- 6 or greater
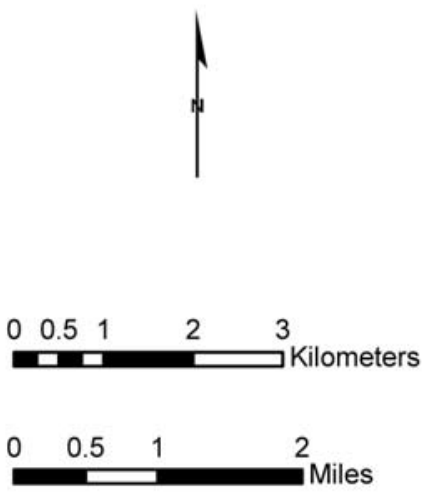

April 28, 2006

YMP-05-042.8

00672DC_021d.ai

Sources: Table A-1; USGS 2000 [DIRS 175858], folder Soilmap6.

NOTE: Depth ranges have been changed from those in Table 6-1 to eliminate overlapping ranges, for the purpose of calculating depth ranges from the original file.

Figure 6-8. Comparison of SOILMAP6.INP Calculated Depths to Borehole Actual Measured Depths 
Data Analysis for Infiltration Modeling: Technical Evaluation of Previous Soil Depth Estimation Methods and Development of Alternate Parameter Values

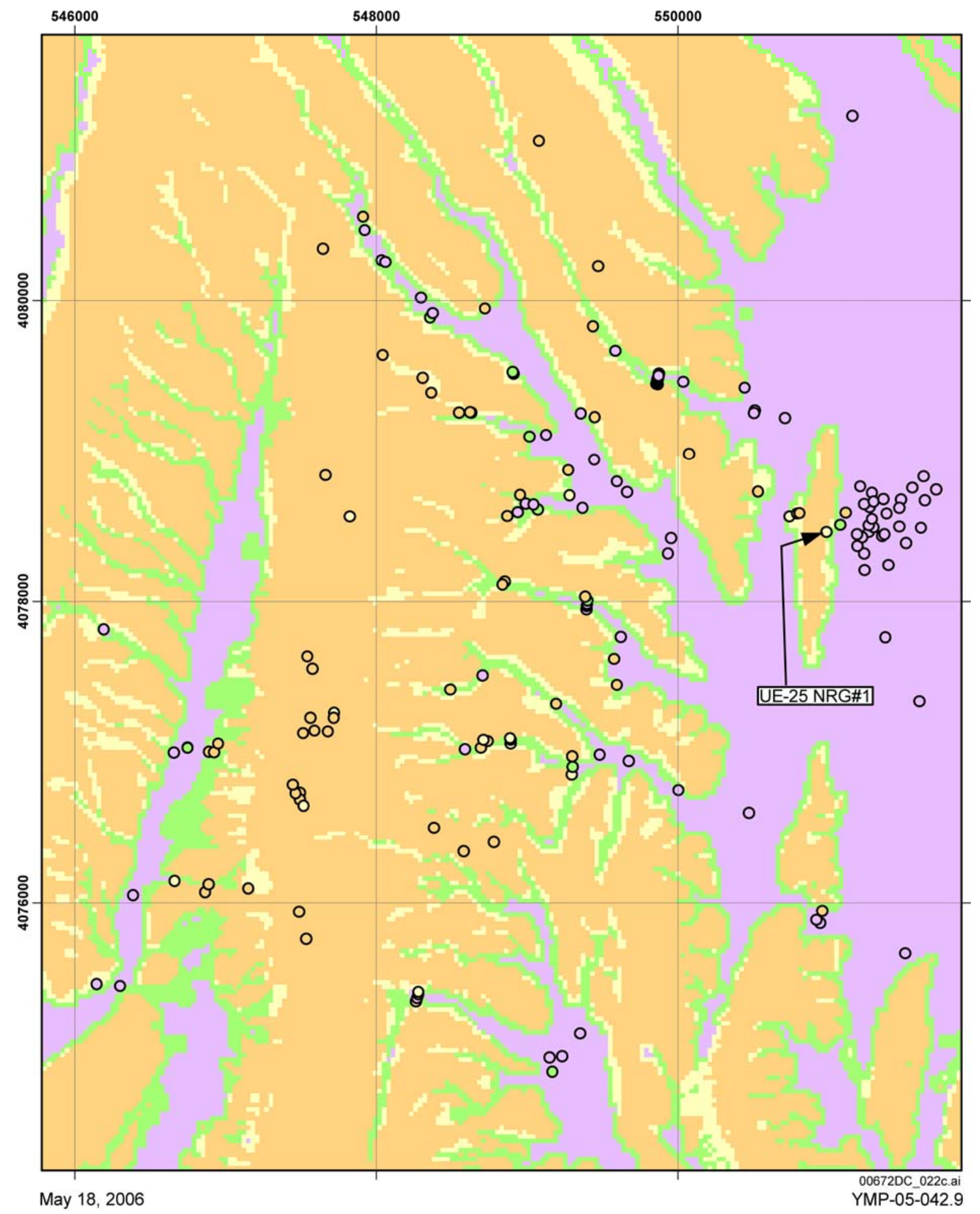

Source: Figure 6-8.

NOTE: Figure 6-8 provides an explanation of symbols.

Figure 6-9. Area of Densest Borehole Coverage (Enlarged) 
Data Analysis for Infiltration Modeling: Technical Evaluation of Previous Soil Depth Estimation Methods and Development of Alternate Parameter Values

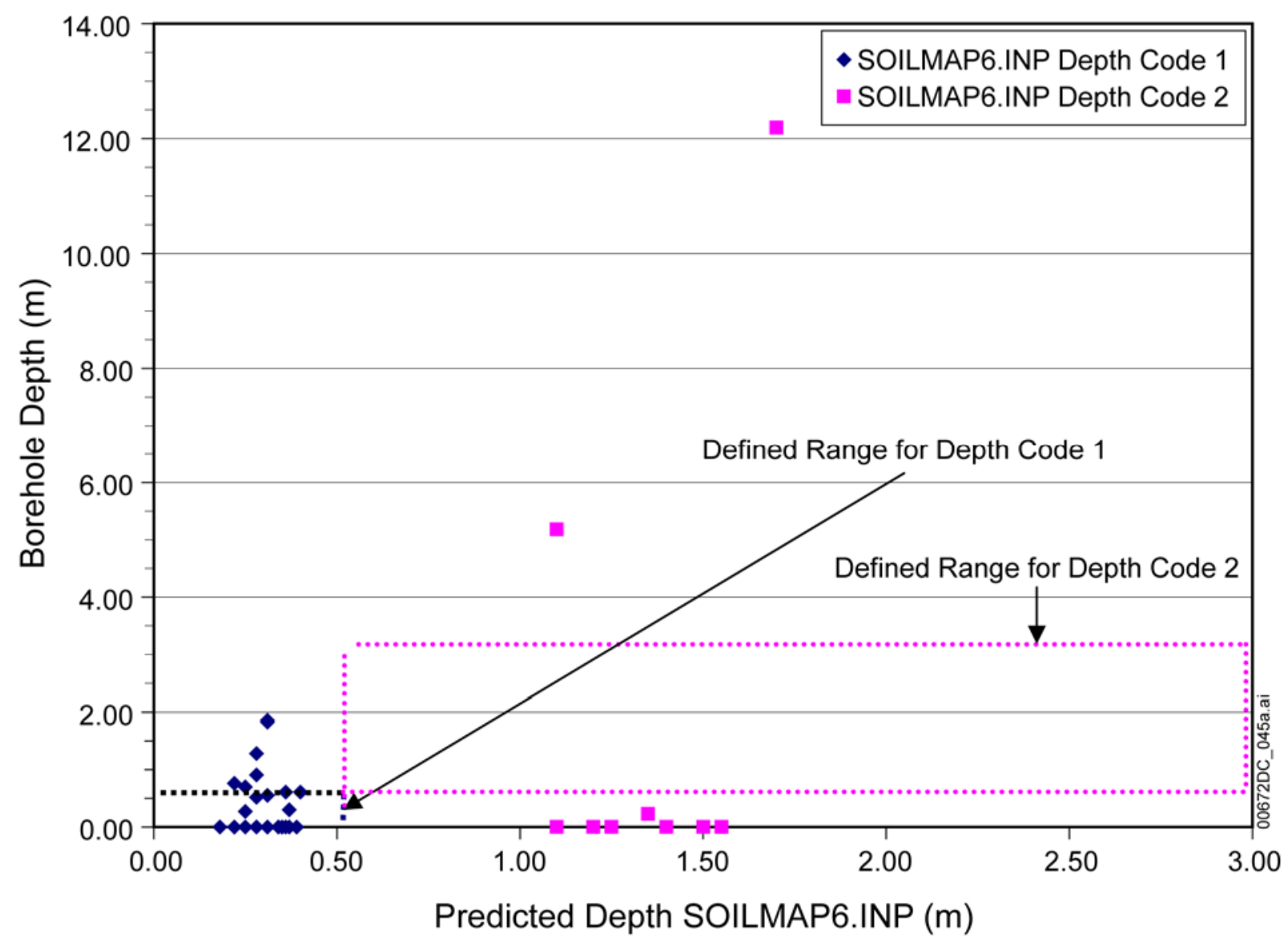

Source: Table 6-4; Appendix B, Section B.1.5 and Final calcs. for report.xls, worksheet 'Soilmap BH diff'.

Figure 6-10. Plot Showing the Relationship between the Soil Depth Measured in Boreholes and the Predicted Soil Depth for the Corresponding Grid Cell in SOILMAP6.INP

Table 6-4. Comparison between Surficial Deposits Thickness Measured in Boreholes and Those Calculated by SOILMAP6.INP

\begin{tabular}{|l|c|c|c|c|}
\hline $\begin{array}{c}\text { Borehole } \\
\text { Identification } \\
\text { Number }\end{array}$ & $\begin{array}{c}\text { Actual Borehole } \\
\text { Alluvium Thickness (m) }\end{array}$ & $\begin{array}{c}\text { SOILMAP6.INP } \\
\text { Depth Code }\end{array}$ & $\begin{array}{c}\text { Estimated } \\
\text { SOILMAP6.INP Soil } \\
\text { Depth (m) }^{(2)}\end{array}$ & $\begin{array}{c}\text { Estimated Soil Depth } \\
\text { Minus Actual Borehole } \\
\text { Thickness (m) }^{\mathbf{a}}\end{array}$ \\
\hline USW G-3 & 0.00 & 1 & 0.18 & 0.18 \\
\hline USW UZ-N27 & 0.00 & 1 & 0.22 & 0.22 \\
\hline USW UZ-N17 & 0.76 & 1 & 0.22 & -0.54 \\
\hline USW UZ-N36 & 0.27 & 1 & 0.25 & -0.02 \\
\hline USW WT-24 & $0.00^{\mathrm{b}}$ & 1 & 0.25 & 0.25 \\
\hline USW UZ-N15 & 0.70 & 1 & 0.25 & -0.45 \\
\hline USW G-2 & 0.00 & 1 & 0.25 & 0.25 \\
\hline USW UZ-N16 & 1.28 & 1 & 0.28 & -1.00 \\
\hline USW UZ-N11 & 0.52 & 0.28 & -0.24 \\
\hline
\end{tabular}


Data Analysis for Infiltration Modeling: Technical Evaluation of Previous Soil Depth Estimation Methods and Development of Alternate Parameter Values

Table 6-4. Comparison between Surficial Deposits Thickness Measured in Boreholes and Those Calculated by SOILMAP6.INP (Continued)

\begin{tabular}{|c|c|c|c|c|}
\hline $\begin{array}{c}\text { Borehole } \\
\text { Identification } \\
\text { Number }\end{array}$ & $\begin{array}{c}\text { Actual Borehole } \\
\text { Alluvium Thickness (m) }\end{array}$ & $\begin{array}{l}\text { SOILMAP6.INP } \\
\text { Depth Code }\end{array}$ & $\begin{array}{c}\text { Estimated } \\
\text { SOILMAP6.INP Soil } \\
\text { Depth }(\mathrm{m})\end{array}$ & $\begin{array}{l}\text { Estimated Soil Depth } \\
\text { Minus Actual Borehole } \\
\text { Thickness (m) }^{\mathrm{a}}\end{array}$ \\
\hline USW H-3 & 0.00 & 1 & 0.28 & 0.28 \\
\hline USW UZ-6 & 0.00 & 1 & 0.28 & 0.28 \\
\hline UE-25 UZ\#5 & 0.91 & 1 & 0.28 & -0.63 \\
\hline UE-25 NRG\#1 & 1.86 & 1 & 0.31 & -1.55 \\
\hline USW UZ-N64 & 0.55 & 1 & 0.31 & -0.24 \\
\hline UE-25 NRG\#5 & $0.00^{\mathrm{b}}$ & 1 & 0.31 & 0.31 \\
\hline USW SD-7 & 0.00 & 1 & 0.34 & 0.34 \\
\hline USW SD-6 & 0.00 & 1 & 0.34 & 0.34 \\
\hline USW H-5 & $0.00^{\mathrm{b}}$ & 1 & 0.34 & 0.34 \\
\hline UE-25 WT\#18 & 0.00 & 1 & 0.36 & 0.36 \\
\hline USW UZ-N55 & 0.00 & 1 & 0.37 & 0.37 \\
\hline USW SD-9 & $0.00^{\mathrm{b}}$ & 1 & 0.37 & 0.37 \\
\hline USW UZ-N62 & 0.30 & 1 & 0.37 & 0.07 \\
\hline UE-25 NRG\#4 & 0.00 & 1 & 0.39 & 0.39 \\
\hline USW UZ-N49 & 0.61 & 1 & 0.36 & -0.25 \\
\hline USW UZ-N65 & 0.00 & 1 & 0.28 & 0.28 \\
\hline USW UZ-N66 & 0.00 & 1 & 0.37 & 0.37 \\
\hline USW UZ-N70 & 0.00 & 1 & 0.31 & 0.31 \\
\hline USW UZ-N71 & 0.00 & 1 & 0.28 & 0.28 \\
\hline USW UZ-N72 & 0.00 & 1 & 0.35 & 0.35 \\
\hline USW UZ-N73 & 0.00 & 1 & 0.34 & 0.34 \\
\hline USW UZ-N74 & 0.00 & 1 & 0.37 & 0.37 \\
\hline USW UZ-N75 & 0.61 & 1 & 0.40 & -0.21 \\
\hline USW UZ-N76 & 0.00 & 1 & 0.37 & 0.37 \\
\hline USW UZ-N78 & 0.00 & 1 & 0.39 & 0.39 \\
\hline USW UZ-N79 & 0.00 & 1 & 0.37 & 0.37 \\
\hline USW UZ-N81 & 1.83 & 1 & 0.31 & -1.52 \\
\hline USW UZ-N93 & 0.00 & 1 & 0.25 & 0.25 \\
\hline USW UZ-N94 & 0.00 & 1 & 0.28 & 0.28 \\
\hline USW UZ-N95 & 0.00 & 1 & 0.31 & 0.31 \\
\hline USW UZ-N96 & 0.61 & 1 & 0.40 & -0.21 \\
\hline UE-25 UZN\#2 & 0.00 & 1 & 0.28 & 0.28 \\
\hline UE-25 UZN\#10 & 0.00 & 1 & 0.28 & 0.28 \\
\hline USW UZ-N32 & 0.00 & 2 & 1.10 & 1.10 \\
\hline USW NRG-7a & 5.18 & 2 & 1.10 & -4.08 \\
\hline UE-25 NRG\#2c & NA & 2 & 1.50 & - \\
\hline UE-25 NRG\#2b & 0.00 & 2 & 1.50 & 1.50 \\
\hline UE-25 NRG\#2 & 0.00 & 2 & 1.55 & 1.55 \\
\hline UE-25 NRG\#2d & NA & 2 & 1.55 & - \\
\hline UE-25 NRG\#3 & $0.00^{b}$ & 2 & 1.55 & 1.55 \\
\hline USW WT-7 & 12.19 & 2 & 1.70 & -10.49 \\
\hline
\end{tabular}


Data Analysis for Infiltration Modeling: Technical Evaluation of Previous Soil Depth Estimation Methods and Development of Alternate Parameter Values

Table 6-4. Comparison between Surficial Deposits Thickness Measured in Boreholes and Those Calculated by SOILMAP6.INP (Continued)

\begin{tabular}{|c|c|c|c|c|}
\hline $\begin{array}{c}\text { Borehole } \\
\text { Identification } \\
\text { Number }\end{array}$ & $\begin{array}{c}\text { Actual Borehole } \\
\text { Alluvium Thickness (m) }\end{array}$ & $\begin{array}{l}\text { SOILMAP6.INP } \\
\text { Depth Code }\end{array}$ & $\begin{array}{c}\text { Estimated } \\
\text { SOILMAP6.INP Soil } \\
\text { Depth }(\mathrm{m})\end{array}$ & $\begin{array}{l}\text { Estimated Soil Depth } \\
\text { Minus Actual Borehole } \\
\text { Thickness }(\mathrm{m})^{\mathrm{a}}\end{array}$ \\
\hline USW UZ-N44 & 0.00 & 2 & 1.20 & 1.20 \\
\hline USW UZ-N80 & 0.00 & 2 & 1.20 & 1.20 \\
\hline USW UZ-N88 & 0.23 & 2 & 1.35 & 1.12 \\
\hline USW UZ-N25 & 0.00 & 2 & 1.40 & 1.40 \\
\hline USW UZ-N26 & 0.00 & 2 & 1.25 & 1.25 \\
\hline USW UZ-N86 & 0.00 & 3 & 4.56 & 4.56 \\
\hline NC-EWDP-18P & 13.72 & 4 & 6.00 & - \\
\hline USW UZ-N59 & 3.99 & 4 & 6.00 & 2.01 \\
\hline UE-25 ONC \#1 & 29.87 & 4 & 6.00 & - \\
\hline USW UZ-N54 & 5.79 & 4 & 6.00 & 0.21 \\
\hline UE-25 UZ\#16 & 10.27 & 4 & 6.00 & - \\
\hline UE-25 RF\#13 & 26.06 & 4 & 6.00 & - \\
\hline UE-25 RF\#22 & 24.38 & 4 & 6.00 & - \\
\hline UE-25 RF\#24 & 6.10 & 4 & 6.00 & - \\
\hline USW UZ-N37 & 11.06 & 4 & 6.00 & - \\
\hline UE-25 UZ\#4 & 11.89 & 4 & 6.00 & - \\
\hline USW UZ-N34 & 15.27 & 4 & 6.00 & - \\
\hline USW UZ-14 & 12.10 & 4 & 6.00 & - \\
\hline UE-25 RF\#18 & 18.29 & 4 & 6.00 & - \\
\hline UE-25 RF\#16 & 16.25 & 4 & 6.00 & - \\
\hline UE-25 RF\#26 & 21.64 & 4 & 6.00 & - \\
\hline UE-25 RF\#14 & 31.03 & 4 & 6.00 & - \\
\hline UE-25 RF\#23 & 19.51 & 4 & 6.00 & - \\
\hline UE-25 RF\#28 & 3.05 & 4 & 6.00 & 2.95 \\
\hline UE-25 RF\#20 & 21.34 & 4 & 6.00 & - \\
\hline UE-25 RF\#15 & 0.00 & 4 & 6.00 & 6.00 \\
\hline UE-25 RF\#19 & 36.58 & 4 & 6.00 & - \\
\hline UE-25 RF\#21 & 33.53 & 4 & 6.00 & - \\
\hline UE-25 RF\#25 & 18.29 & 4 & 6.00 & - \\
\hline UE-25 RF\#29 & 25.91 & 4 & 6.00 & - \\
\hline UE-25 RF\#17 & 28.16 & 4 & 6.00 & - \\
\hline USW G-1 & 18.29 & 4 & 6.00 & - \\
\hline UE-25 p\#1 & 39.01 & 4 & 6.00 & - \\
\hline UE-25 J\#13 & 132.59 & 4 & 6.00 & - \\
\hline USW a\#1 & 9.14 & 4 & 6.00 & - \\
\hline UE-25 a\#5 & 27.43 & 4 & 6.00 & - \\
\hline UE-25 a\#6 & 6.10 & 4 & 6.00 & - \\
\hline UE-25 a\#7 & 50.29 & 4 & 6.00 & - \\
\hline UE-25 b\#1 & 47.55 & 4 & 6.00 & - \\
\hline
\end{tabular}


Data Analysis for Infiltration Modeling: Technical Evaluation of Previous Soil Depth Estimation Methods and Development of Alternate Parameter Values

Table 6-4. Comparison between Surficial Deposits Thickness Measured in Boreholes and Those Calculated by SOILMAP6.INP (Continued)

\begin{tabular}{|c|c|c|c|c|}
\hline $\begin{array}{c}\text { Borehole } \\
\text { Identification } \\
\text { Number }\end{array}$ & $\begin{array}{c}\text { Actual Borehole } \\
\text { Alluvium Thickness (m) }\end{array}$ & $\begin{array}{l}\text { SOILMAP6.INP } \\
\text { Depth Code }\end{array}$ & $\begin{array}{c}\text { Estimated } \\
\text { SOILMAP6.INP Soil } \\
\text { Depth }(\mathrm{m})\end{array}$ & $\begin{array}{l}\text { Estimated Soil Depth } \\
\text { Minus Actual Borehole } \\
\text { Thickness (m) }^{\mathrm{a}}\end{array}$ \\
\hline UE-25 c\#2 & 21.31 & 4 & 6.00 & - \\
\hline UE-25 c\#3 & 24.41 & 4 & 6.00 & - \\
\hline USW UZ-1 & 12.19 & 4 & 6.00 & - \\
\hline USW WT-1 & 9.14 & 4 & 6.00 & - \\
\hline UE-25 WT\#6 & 51.82 & 4 & 6.00 & - \\
\hline USW WT-10 & 18.29 & 4 & 6.00 & - \\
\hline USW WT-11 & 12.19 & 4 & 6.00 & - \\
\hline UE-25 WT\#12 & 18.29 & 4 & 6.00 & - \\
\hline UE-25 WT\#13 & 67.06 & 4 & 6.00 & - \\
\hline UE-25 WT\#14 & 32.61 & 4 & 6.00 & - \\
\hline UE-25 WT\#15 & 64.01 & 4 & 6.00 & - \\
\hline UE-25 WT\#16 & 41.76 & 4 & 6.00 & - \\
\hline UE-25 WT\#17 & 9.14 & 4 & 6.00 & - \\
\hline UE-25 J\#12 & 156.97 & 4 & 6.00 & - \\
\hline UE-25 JF \#3 & 148.44 & 4 & 6.00 & - \\
\hline USW UZ-N45 & 10.67 & 4 & 6.00 & - \\
\hline USW UZ-N47 & 10.06 & 4 & 6.00 & - \\
\hline USW UZ-N67 & 5.79 & 4 & 6.00 & 0.21 \\
\hline USW UZ-N68 & 15.24 & 4 & 6.00 & - \\
\hline USW UZ-N69 & 8.23 & 4 & 6.00 & - \\
\hline USW UZ-N82 & 6.70 & 4 & 6.00 & - \\
\hline USW UZ-N84 & 6.09 & 4 & 6.00 & - \\
\hline USW UZ-N87 & 5.79 & 4 & 6.00 & 0.21 \\
\hline USW UZ-N89 & 10.06 & 4 & 6.00 & - \\
\hline USW UZ-N90 & 9.91 & 4 & 6.00 & - \\
\hline UE-25 UZN\#1 & 8.32 & 4 & 6.00 & - \\
\hline UE-25 UZN\#7 & 12.34 & 4 & 6.00 & - \\
\hline UE-25 UZN\#8 & 12.19 & 4 & 6.00 & - \\
\hline UE-25 UZN\#9 & 10.67 & 4 & 6.00 & - \\
\hline UE-25 UZN\#12 & 13.72 & 4 & 6.00 & - \\
\hline UE-25 UZN\#13 & 13.41 & 4 & 6.00 & - \\
\hline UE-25 UZN\#14 & 13.41 & 4 & 6.00 & - \\
\hline UE-25 UZN\#18 & 17.07 & 4 & 6.00 & - \\
\hline UE-25 UZN\#21 & 8.38 & 4 & 6.00 & - \\
\hline UE-25 UZN\#22 & 5.94 & 4 & 6.00 & 0.06 \\
\hline UE-25 UZN\#56 & 17.07 & 4 & 6.00 & - \\
\hline UE-25 UZN\#97 & 17.68 & 4 & 6.00 & - \\
\hline UE-29 UZN\#92 & 17.68 & 4 & 6.00 & - \\
\hline USW UZ-N61 & 2.99 & 5 & 3.92 & 0.93 \\
\hline USW NRG-7 & 0.00 & 5 & 3.92 & 3.92 \\
\hline USW UZ-N33 & 3.99 & 5 & 4.08 & 0.09 \\
\hline
\end{tabular}


Data Analysis for Infiltration Modeling: Technical Evaluation of Previous Soil Depth Estimation Methods and Development of Alternate Parameter Values

Table 6-4. Comparison between Surficial Deposits Thickness Measured in Boreholes and Those Calculated by SOILMAP6.INP (Continued)

\begin{tabular}{|c|c|c|c|c|}
\hline $\begin{array}{c}\text { Borehole } \\
\text { Identification } \\
\text { Number }\end{array}$ & $\begin{array}{c}\text { Actual Borehole } \\
\text { Alluvium Thickness (m) }\end{array}$ & $\begin{array}{l}\text { SOILMAP6.INP } \\
\text { Depth Code }\end{array}$ & $\begin{array}{c}\text { Estimated } \\
\text { SOILMAP6.INP Soil } \\
\text { Depth }(\mathrm{m})\end{array}$ & $\begin{array}{l}\text { Estimated Soil Depth } \\
\text { Minus Actual Borehole } \\
\text { Thickness (m) }^{\mathrm{a}}\end{array}$ \\
\hline USW UZN\#63 & 2.62 & 5 & 4.24 & 1.62 \\
\hline USW UZ-7a & 0.00 & 5 & 4.40 & 4.40 \\
\hline USW SD-12 & 0.00 & 5 & 4.40 & 4.40 \\
\hline UE-25 a\#4 & 9.14 & 5 & 4.40 & -4.74 \\
\hline USW UZ-N35 & 3.60 & 5 & 4.56 & 0.96 \\
\hline USW NRG-6 & 0.00 & 5 & 4.56 & 4.56 \\
\hline USW UZ-N38 & 5.46 & 5 & 4.56 & -0.90 \\
\hline USW UZ- N53 & 0.64 & 5 & 4.88 & 4.24 \\
\hline USW G-4 & 9.14 & 5 & 4.88 & -4.26 \\
\hline USW H-6 & 9.11 & 5 & 4.88 & -4.23 \\
\hline USW WT-2 & 18.29 & 5 & 4.88 & -13.41 \\
\hline USW H-1 & 0.00 & 5 & 5.04 & 5.04 \\
\hline USW H-4 & 0.00 & 5 & 5.04 & 5.04 \\
\hline UE-25 NRG\#2a & 2.07 & 5 & 5.20 & 3.13 \\
\hline USW UZ-N31 & 0.00 & 5 & 5.20 & 5.20 \\
\hline UE-25 WT\#3 & 3.35 & 5 & 5.36 & 2.01 \\
\hline UE-25 WT\#4 & 15.54 & 5 & 5.36 & -10.18 \\
\hline USW UZ-N57 & 0.37 & 5 & 5.52 & 5.15 \\
\hline USW UZ-N58 & 6.74 & 5 & 5.52 & -1.22 \\
\hline UE-25 c\#1 & 0.00 & 5 & 5.68 & 5.68 \\
\hline USW UZ-N40 & 0.88 & 5 & 4.72 & 3.84 \\
\hline USW UZ-N41 & 4.88 & 5 & 5.52 & 0.64 \\
\hline USW UZ-N42 & 0.00 & 5 & 4.40 & 4.40 \\
\hline USW UZ-N43 & 8.23 & 5 & 4.72 & -3.51 \\
\hline USW UZ-N46 & 0.00 & 5 & 4.88 & 4.88 \\
\hline USW UZ-N48 & 0.00 & 5 & 4.72 & 4.72 \\
\hline USW UZ-N50 & 2.74 & 5 & 3.44 & 0.70 \\
\hline USW UZ-N51 & 4.27 & 5 & 4.88 & 0.61 \\
\hline USW UZ-N52 & 2.13 & 5 & 4.88 & 2.75 \\
\hline USW UZ-N77 & 11.58 & 5 & 5.52 & -6.06 \\
\hline USW UZ-N83 & 0.00 & 5 & 4.72 & 4.72 \\
\hline USW UZ-N98 & 0.30 & 5 & 4.08 & 3.78 \\
\hline USW UZ-N24 & 0.15 & 5 & 4.08 & 3.93 \\
\hline UE-25 UZN\#3 & 2.74 & 5 & 4.40 & 1.66 \\
\hline UE-25 UZN\#4 & 7.47 & 5 & 4.40 & -3.07 \\
\hline UE-25 UZN\#5 & 13.56 & 5 & 4.40 & -9.16 \\
\hline UE-25 UZN\#6 & 12.04 & 5 & 4.40 & -7.64 \\
\hline UE-25 UZN\#19 & 6.86 & 5 & 4.56 & -2.30 \\
\hline UE-25 UZN\#20 & 11.89 & 5 & 5.36 & -6.53 \\
\hline UE-25 UZN\#23 & 0.00 & 5 & 4.56 & 4.56 \\
\hline UE-25 UZN\#29 & 0.30 & 5 & 4.88 & 4.58 \\
\hline
\end{tabular}


Table 6-4. Comparison between Surficial Deposits Thickness Measured in Boreholes and Those Calculated by SOILMAP6.INP (Continued)

\begin{tabular}{|l|c|c|c|c|}
\hline $\begin{array}{c}\text { Borehole } \\
\text { Identification } \\
\text { Number }\end{array}$ & $\begin{array}{c}\text { Actual Borehole } \\
\text { Alluvium Thickness (m) }\end{array}$ & $\begin{array}{c}\text { Estimated } \\
\text { SOILMAP6.INP } \\
\text { Depth Code }\end{array}$ & $\begin{array}{c}\text { Estimated Soil Depth } \\
\text { SOILMAP6.INP Soil } \\
\text { Depth (m) }\end{array}$ & $\begin{array}{c}\text { Minus Actual Borehole } \\
\text { Thickness (m) }\end{array}$ \\
\hline UE-25 UZN\#30 & 0.38 & 5 & 4.24 & 3.86 \\
\hline UE-25 UZN\#60 & 8.08 & 5 & 5.36 & -2.72 \\
\hline
\end{tabular}

Sources: Table A-1; USGS 2000 [DIRS 175858], folder Soilmap6; Appendix B, Section B.1.5 for Excel@ calculation.

a A dash (-) in the last column indicates that either the actual borehole alluvial thickness was not provided or both the actual and estimated soil thickness (depth) are not less than $6 \mathrm{~m}$.

b Zero values are shown on the borehole log, but inspection of the pad cut indicated that soil was present (Section 6.1.2).

$\mathrm{NA}=$ not applicable.

Several boreholes fall into Soil Depth Code 5 (Table 6-4), which has the greatest soil depth variation. Soil Depth Code 5 was created using the software routines GEOMOD4 V1.0 and SOILMAP6 V1.0 (BSC 2004 [DIRS 170007], Appendices F and G) to provide an intermediate-depth buffer cell between cells that were classed as shallow soils and deep alluvium on the basis of soil type. Therefore, the locations of these intermediate depth cells represent an area of rapid depth change by definition. This is also the type of terrain where drill pad construction practices may affect the comparison. The large depth variation in this code, with an average difference of about plus or minus $4 \mathrm{~m}$, indicates that the methods used to create the file result in larger errors where soil depths change over small horizontal distances.

Because depths are not calculated for depths greater than $6 \mathrm{~m}$ in SOILMAP6.INP (USGS 2000 [DIRS 175858], folder Soilmap6), and all cells in Soil Depth Code 4 are assigned a uniform value of $6 \mathrm{~m}$, the only comparison that can be made for Soil Depth Code 4 is whether a borehole indicates a depth greater than $6 \mathrm{~m}$. Only six of the 91 boreholes in Soil Depth Code 4 indicate depths shallower than $6 \mathrm{~m}$. These six boreholes are located near the contact with shallower soil depth codes and are probably indicative of the difficulty in precisely defining contacts between soil depth codes.

\subsection{ALTERNATE SOIL DEPTH EVALUATION}

Upon review (Section 6.1.2), it was determined that an alternate soil depth file, other than SOILMAP6.INP (USGS 2000 [DIRS 175858], folder Soilmap6), should be generated and documented for use in future infiltration modeling. This alternate approach was taken because of transparency and traceability issues raised by the uncertainty in how the equations for calculating depth were derived, by questions about how end points of the depth classes correspond to ranges calculated by depth equations in the infiltration model report (BSC 2004 [DIRS 170007]), and because of poor corroboration by available data sets of the soil depth calculations. Therefore, an alternate soil depth file has been created, based on a different and simplified approach compared to that used in SOILMAP6.INP (USGS 2000 [DIRS 175858], folder Soilmap6). This alternate soil depth file is based on the same soil map (Figure 6-1) used to prepare SOILMAP6.INP. 


\subsubsection{Summary and Evaluation of Soil Depth Data}

The grouping of soil types used in DTN: GS960408312212.005 [DIRS 146299], ACC: JOL.19961118.0098 (Figure 6-1 and Table 6-2) are used as a starting point for evaluation. The general soil depth classes interpreted to correspond to these soil types (Table 6-2) were used to generate the three combined soil depth classes illustrated on Figure 6-2. For this grouping, cells belonging to the Disturbed Ground classification, such as for roads and drill pads, were assigned to the same general depth class as surrounding, undisturbed cells, on the assumption that the soil depth under these features would not differ greatly from the surrounding area. Roads and drill pads are a small percentage of the total area, are often located over thicker alluvial areas, and are generally not much higher or lower than the surrounding area.

For this evaluation, two additional soil depth classes were added to the three that are shown on Figure 6-2. As discussed in the following paragraphs, the deep soil class shown on Figure 6-2 contains soils that can range in depth from a few meters to over $150 \mathrm{~m}$. The subdivision of the deep soil class into two soil depth classes provides better definition of the shallower part of this range. This is desirable because previous versions of the infiltration model (BSC 2004 [DIRS 170007]); USGS 2000 [DIRS 123650]; USGS 2001 [DIRS 154674]; USGS 2001 [DIRS 160355]; USGS 2003 [DIRS 166518]) have indicated that shallow soils may transmit water to the soil-bedrock interface, whereas deeper soils may not (BSC 2004 [DIRS 170007], pp. 5-2 and 6-2). Other estimates of percolation also indicate little or no deep percolation below depths of about 6 to $8 \mathrm{~m}$; for example, percolation depths based on chloride accumulation beneath native vegetation in the Amargosa Desert south of Yucca Mountain (Stonestrom et al. 2003 [DIRS 165862], p. 16). Therefore, a fourth class was added by subdividing the deep soil class into a moderately deep soil class and a very deep soil class to add more definition to the deep soils that may be significant in future modeling.

The boundaries for the moderately deep soil class were based on a review of the large number of boreholes in the vicinity of the proposed Waste Handling Building in Midway Valley, as shown in Figure 6-11.

Boreholes were projected to an east-west line, perpendicular to the strike of the structural trend in the area, to evaluate soil depth trends in this area. Distances (Figure 6-11) were measured eastward from the location of UE-25 NRG\#1 (Figure 6-9) at the portal of the Exploratory Studies Facility on Exile Hill. Boreholes within about $200 \mathrm{~m}$ of this point are located in the shallow and intermediate depth classes. Boreholes at a greater distance are in alluvium that would be assigned to the newly defined moderately deep soil class or to the very deep soil class. Figure 6-11 shows that depth generally increases in a linear fashion with distance beyond $200 \mathrm{~m}$, which is the approximate boundary between the shallow or intermediate depth classes and the moderately deep soil class.

Given that there is no natural break in the depth-distance trend to define a subdivision, the moderately deep soil class was defined by arbitrarily setting an upper limit that extends 10 cells, or $300 \mathrm{~m}$, out from the boundaries of the shallow or intermediate depth classes using ARCINFO (Figure 6-12). This definition creates a zone of sufficient lateral dimensions to be significant in modeling and was deliberately defined to include depths that are expected to range from near zero meters to about $40 \mathrm{~m}$. 


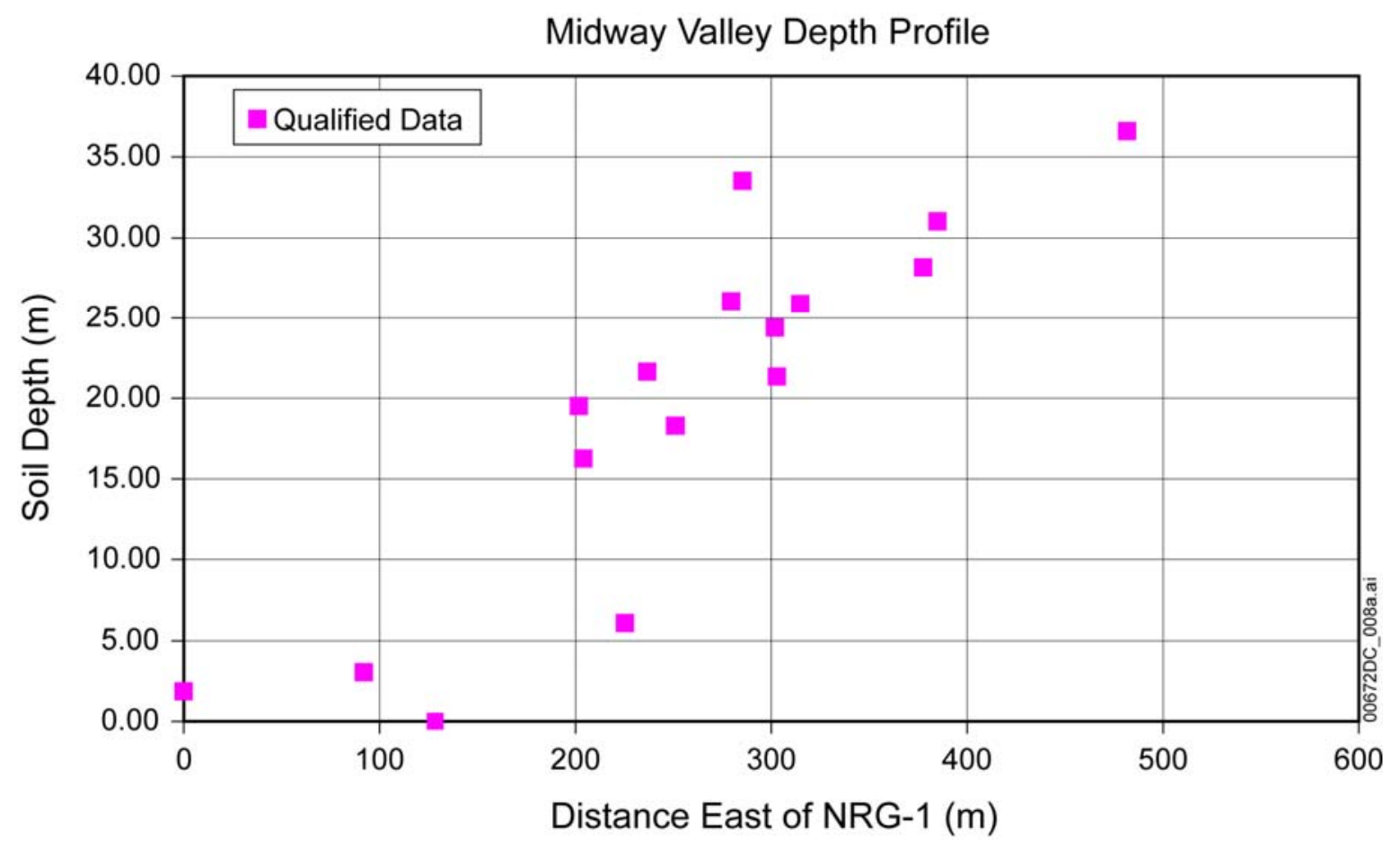

Sources: Table A-1; Appendix B, Section B.1.7 and Final calcs. for report.xls, worksheet 'Midway Valley depth'.

Figure 6-11. Data of Borehole Soil Depth Near the Proposed Waste Handling Building (Midway Valley); Projected to an East-West Line

The depth range was selected to include the soil depth limit for infiltration defined by previous models. Previous infiltration models were based on an assumption that soils deeper than $6 \mathrm{~m}$ do not permit infiltration of water to the soil-bedrock contact, except in some channels, because the soils have sufficient storage capacity to retain precipitation in the root zone where it is subject to evapotranspiration. Therefore, previous models had assigned a constant depth of $6 \mathrm{~m}$ to all soils of greater depth (BSC 2004 [DIRS 170007], pp. 5-2 and 6-2). For this evaluation, a somewhat broader potential depth range was selected to incorporate the uncertainties inherent in assigning an areal distribution to the moderately deep soil class and any uncertainties in the infiltration depth limit.

The fifth soil depth class was added (Figure 6-12) to create a separate class for the exposed bedrock shown as Soil Type Unit 8 (Figure 6-1), making it possible to differentiate between areas with some soil thickness, such as the shallow soils-colluvium class, and areas devoid of surficial deposits of sufficient size to be mapped. Depth classes (Figure 6-12) are included in the alternate soil depth file, output DTN: MO0606SPASDFIM.005. This soil depth file uses the same map area and soil groupings based on the soil types given in DTN: GS960408312212.005 [DIRS 146299], ACC: MOL.19961118.0100, and the same $30 \times 30 \mathrm{~m}$ grid system used in previous versions of the infiltration model (BSC 2004 [DIRS 170007]); USGS 2000 [DIRS 123650]; USGS 2001 [DIRS 154674]; USGS 2001 [DIRS 160355]; USGS 2003 [DIRS 166518]). 
Data Analysis for Infiltration Modeling: Technical Evaluation of Previous Soil Depth Estimation Methods and Development of Alternate Parameter Values

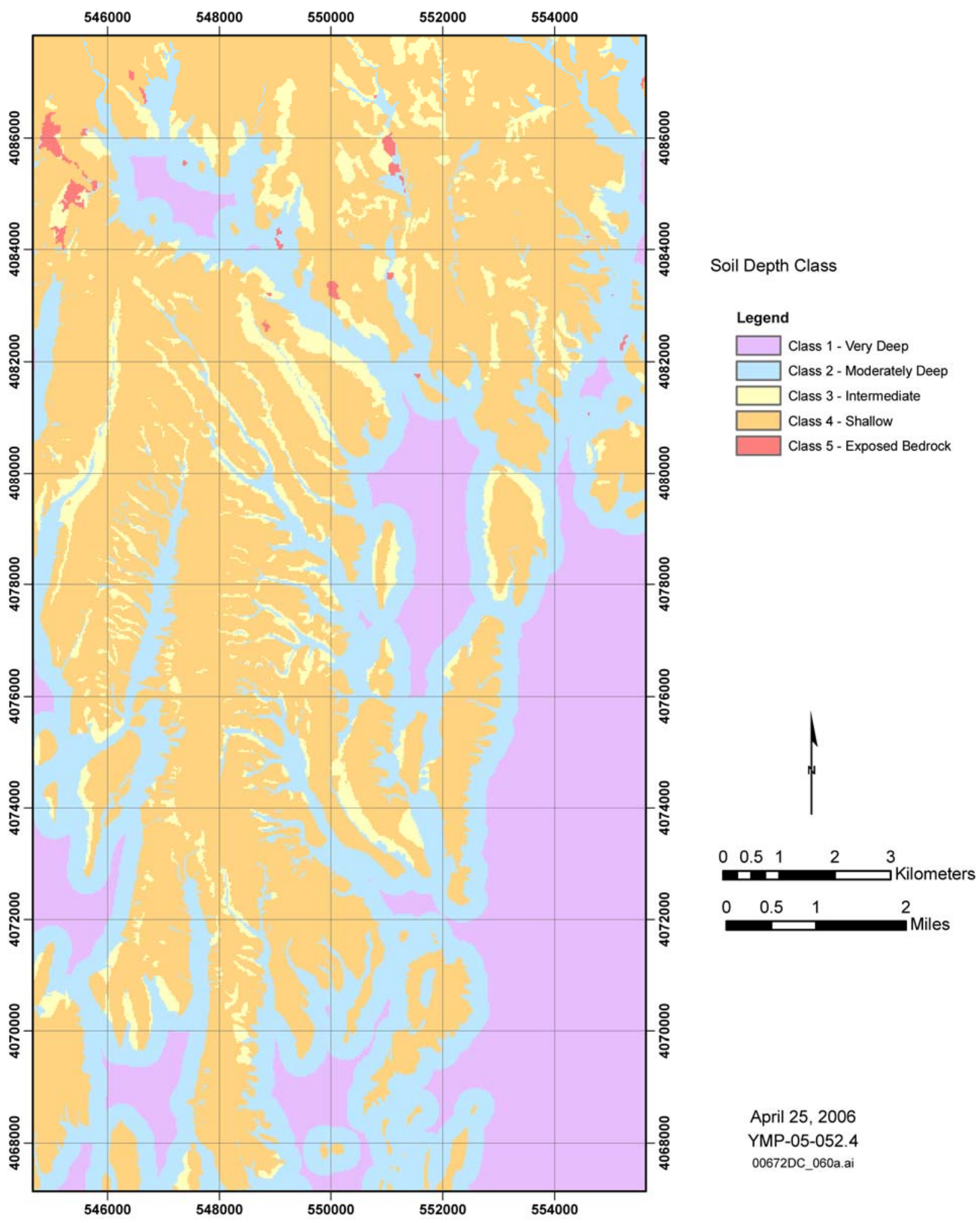

Source: Output DTN: MO0606SPASDFIM.005.

NOTE: Very deep, intermediate, and shallow class data are from Figure 6-2.

Figure 6-12. Graphical Representation of Depth Classes Used for Alternate Soil Depth Evaluation 
Each grid cell in the model area is assigned a soil depth class. For ease in implementing the model, a numerical designator is used in the file, rather than a name to identify the soil depth class. The five numerical designators are:

1. Very deep alluvium

2. Moderately deep alluvium

3. Intermediate depth soils

4. Shallow soils-colluvium

5. Exposed bedrock.

Depth ranges were then assigned to the five soil depth classes and then soil depth distributions were developed for each class; general depth ranges for the shallow and intermediate depth classes were given in reports, as discussed in the following paragraphs, that accompany soil maps by Lundstrom et al., as noted in Table 6-5.

Table 6-5. Interpreted Soil Thickness Ranges

\begin{tabular}{|c|c|c|c|c|c|c|}
\hline Class & $\begin{array}{c}\text { Soil Type } \\
\text { Unit } \\
\text { Number }^{a}\end{array}$ & $\begin{array}{c}\text { Soil } \\
\text { Classification }^{\mathrm{a}}\end{array}$ & $\begin{array}{c}\text { Soil } \\
\text { Type } \\
b, c, d, e\end{array}$ & Soil Name ${ }^{b, c, d, e}$ & $\begin{array}{c}\text { Minimum } \\
\text { Thickness } \\
(\mathrm{m})\end{array}$ & $\begin{array}{c}\text { Maximum } \\
\text { Thickness } \\
(\mathrm{m})\end{array}$ \\
\hline $\begin{array}{l}\text { Soil Depth } \\
\text { Class } 4 \text { - Shallow } \\
\text { Soil/Colluvium }\end{array}$ & 5 & $\begin{array}{l}\text { Lithic } \\
\text { Haplocambids }\end{array}$ & $\mathrm{cu}$ & Undivided Colluvium & $0.1^{f}$ & $1^{b, c, d, e}$ \\
\hline $\begin{array}{l}\text { Soil Depth } \\
\text { Class } 4 \text { - Shallow } \\
\text { Soil/Colluvium }\end{array}$ & 7 & $\begin{array}{l}\text { Lithic } \\
\text { Haplargids } \\
\end{array}$ & $\mathrm{rc}$ & Summit Colluvium & $0.1^{f}$ & $1^{c, d}$ \\
\hline $\begin{array}{l}\text { Soil Depth Class } 3 \\
\text { - Intermediate } \\
\text { Depth Soils }\end{array}$ & 9 & $\begin{array}{l}\text { Typic } \\
\text { Calciargids }\end{array}$ & $\mathrm{cf}$ & $\begin{array}{l}\text { Footslope Colluvium } \\
\text { and Alluvium }\end{array}$ & $0.5^{\mathrm{b}, \mathrm{c}, \mathrm{d}, \mathrm{e}}$ & $>3^{\mathrm{e}}$ \\
\hline
\end{tabular}

a DTN: GS960408312212.005 [DIRS 146299], ACC: JOL.19961118.0098

b DTN: GS940108315142.004 [DIRS 160344], ACC: MOL.20021001.0238, RIS p. 13

c DTN: GS940108315142.005 [DIRS 160345], ACC: MOL.20021203.0409, RIS pp. 20 and 22

d DTN: GS940708315142.008 [DIRS 160346], ACC: MOL.20021001.0261, RIS pp. 17 and 19

e DTN: GS950408315142.004 [DIRS 160347], ACC: MOL.20020827.0355, RIS pp. 27 and 29

$f$ This is the minimum thickness inferred from maps (footnotes $b$ to $e$ ) that show nearly all upland surfaces to be soil covered (i.e., non-zero) rather than bare rock (Soil Type r). Minimum thickness is also determined by measurements in DTN: GS011208312212.004 [DIRS 176317], Table S02086_001, for soil depth in the "Tpc" and "stone st" deposit units, and corroborated by soil depth measurements on side slopes and ridge crests (Sanchez 2006 [DIRS 176569], pp. 59 and 62 to 68).

Direct field measurements of soil depth are given in DTN: GS011208312212.004 [DIRS 176317], Table S02086_001, and are used for the shallow soil classes (Table 6-5). Other sources of qualified information are borehole data listed in Section 6.2, pages 6-34 and 6-38 to $6-41$. 
For this evaluation, a distribution was used to characterize the depth variability in all but the exposed bedrock soil depth class. This approach was used because of the limited data available on soil depth. This approach differs from the previous approach used in the infiltration model (BSC 2004 [DIRS 170007]) in that the evaluation does not attempt to provide a numerical correlation of soil depth with other factors, such as topographic slope. Such schemes were not employed because of the absence of qualified and verified data that directly tie soil depth values to topographic slope. This approach, however, does include the influence of topography on soil depth via the mapped soil depth classes as presented (Figure 6-2), which are based in part on topography and soil type.

Because the depth values for a soil depth class are presented as a distribution rather than being based on the attributes of a particular cell, such as slope, as was done in SOILMAP6.INP (USGS 2000 [DIRS 175858], folder Soilmap6), this evaluation is not intended to predict the actual depth values within specific grid cells based on their properties. Use of the distribution is intended to characterize the general range of depths that might be encountered within a soil depth class covering a large area of many cells. With this approach, cell values may be assigned using various methods, such as sampling from the distribution or a mean value based on the distribution, so that an area of many cells is representative of the variation seen in the point measurements found in the data sources. These larger areas should then represent the variation seen in the distribution in the model.

This approach is considered adequate for modeling purposes when the uncertainty in any estimate of this type is considered. In this case, the uncertainty consists of two components: the measurement uncertainty resulting from the small number of direct measurements, such as from boreholes, and the inherent variability in the soil thickness across the area where the actual thickness may vary significantly, even within a single grid cell.

The exposed bedrock of Soil Depth Class 5 is described as volcanic bedrock with small areas of thin colluvium in the original surficial deposits mapping; listed as Lundstrom Soil Classification "r" on Table 6-2 (DTN: GS940108315142.004 [DIRS 160344], ACC: MOL.20021001.0238). Based on this description, cells in this depth class should be assigned a zero soil depth.

The distribution for the shallow soils of Soil Depth Class 4 is based on the direct field measurements in DTN: GS011208312212.004 [DIRS 176317], Table S02086_001, and is corroborated by the range of 0.1 to $1 \mathrm{~m}$ estimated from soil mapping (Table 6-5) and the qualified data for boreholes located in cells assigned to this class. A lognormal distribution was selected to represent Soil Depth Class 4 because DTN: GS011208312212.004 [DIRS 176317], Table S02086_001, indicates that the soil depth for this class is better described by a lognormal distribution (Section B.1.8). This data set represents field measurements of soil depth from natural and artificial exposures in the infiltration model area. Values with "Deposit Unit" designations of "Tpc" and "stone st" in DTN: GS011208312212.004 [DIRS 176317], Table S02086_001, are interpreted as belonging to Soil Depth Class 4 (Table 6-6). The distribution of these data is illustrated (Figure 6-13) in both linear and $\log _{e}$ transformed format. 
Data Analysis for Infiltration Modeling: Technical Evaluation of Previous Soil Depth Estimation Methods and Development of Alternate Parameter Values

Table 6-6. Field Measurements of Soil Depth for Soil Depth Class 4

\begin{tabular}{|c|c|c|}
\hline General Location & Soil Depth (m) & LN Depth (m) \\
\hline- & 0.05 & -2.99573 \\
\hline - & 0.10 & -2.30259 \\
\hline Alice Crest & 0.10 & -2.30259 \\
\hline ridge top & 0.10 & -2.30259 \\
\hline UZ-14 & 0.10 & -2.30259 \\
\hline - & 0.10 & -2.30259 \\
\hline Alice & 0.12 & -2.12026 \\
\hline - & 0.15 & -1.89712 \\
\hline - & 0.17 & -1.77196 \\
\hline Isolation Ridge & 0.20 & -1.60944 \\
\hline Alice & 0.20 & -1.60944 \\
\hline - & 0.20 & -1.60944 \\
\hline ridge top & 0.20 & -1.60944 \\
\hline Split Wash & 0.20 & -1.60944 \\
\hline Pagany Wash & 0.20 & -1.60944 \\
\hline - & 0.20 & -1.60944 \\
\hline Split Wash & 0.25 & -1.38629 \\
\hline Split Wash & 0.25 & -1.38629 \\
\hline - & 0.25 & -1.38629 \\
\hline Yucca Wash & 0.25 & -1.38629 \\
\hline Drill Hole Wash & 0.30 & -1.20397 \\
\hline Antler Ridge & 0.30 & -1.20397 \\
\hline - & 0.35 & -1.04982 \\
\hline Alice & 0.40 & -0.91629 \\
\hline Fran Ridge & 0.40 & -0.91629 \\
\hline Bow Ridge & 0.40 & -0.91629 \\
\hline Trench 14 & 0.40 & -0.91629 \\
\hline Antler Ridge & 0.45 & -0.79851 \\
\hline ESF south portal & 0.50 & -0.69315 \\
\hline UZ-7A & 0.50 & -0.69315 \\
\hline NRG-5 & 0.50 & -0.69315 \\
\hline Alice & 0.75 & -0.28768 \\
\hline UZ-7A & 1.00 & 0.00000 \\
\hline Split Wash & 3.00 & 1.09861 \\
\hline Yucca Wash & 3.00 & 1.09861 \\
\hline \multicolumn{3}{|c|}{ Sample Statistics } \\
\hline LN Mean $(\mathrm{m})$ & & -1.29 \\
\hline LN Standard Deviation (m) & & 0.88 \\
\hline Median (m) (not log transformed) & & 0.25 \\
\hline
\end{tabular}

Sources: DTN: GS011208312212.004 [DIRS 176317], Table S02086_001; Appendix B, Section B.1.8 for Excel® calculations.

$\mathrm{LN}=$ natural logarithm. 
Data Analysis for Infiltration Modeling: Technical Evaluation of Previous Soil Depth Estimation Methods and Development of Alternate Parameter Values
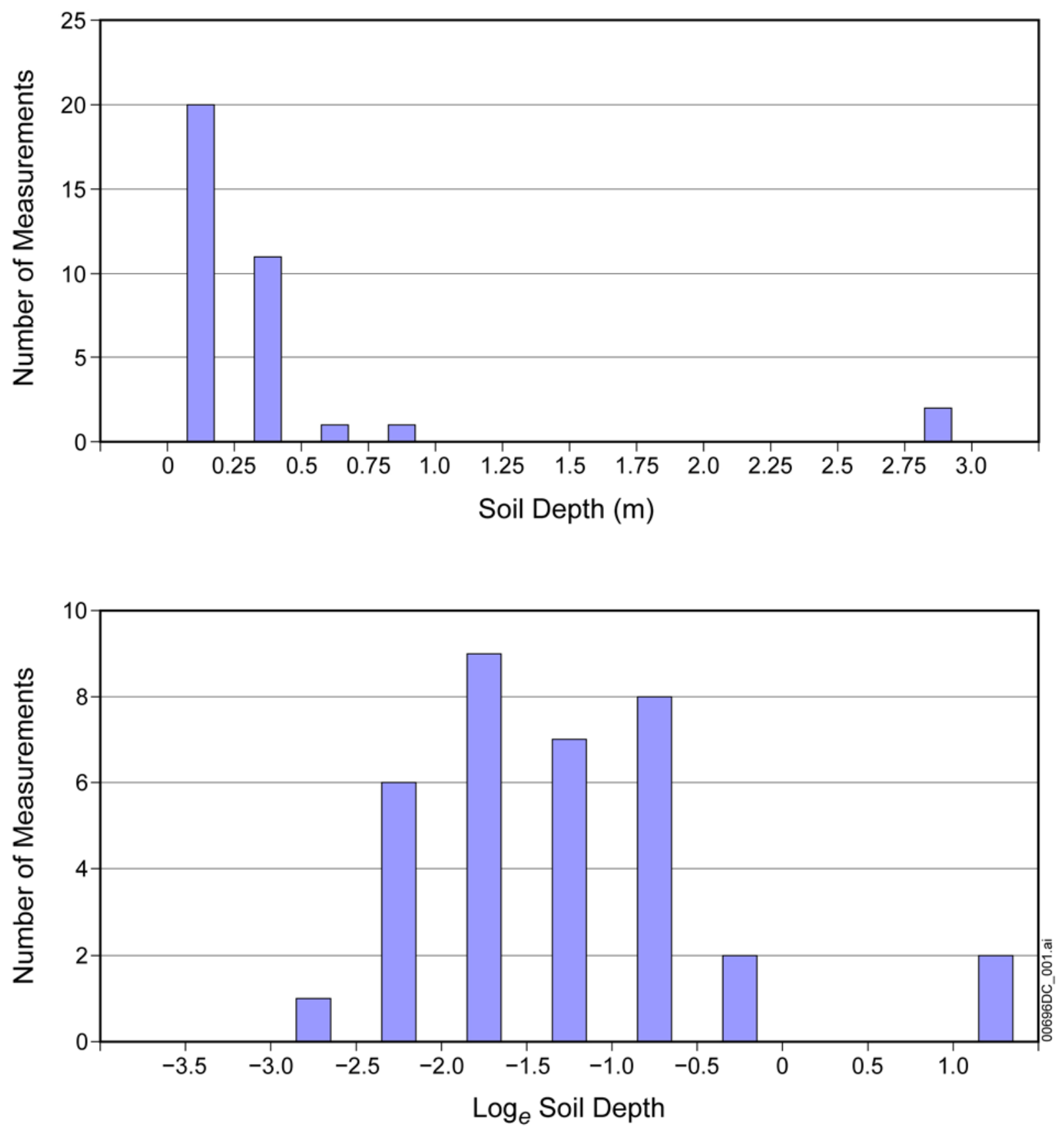

Sources: Table 6-6; Appendix B, Section B.1.8 and Taylor soil depth.xls, worksheet 'Wtest'.

NOTE: $\quad$ Soil Depth $(m)$ shows field measurements, while $\log _{e}$ Soil Depth shows transformed measurements.

Figure 6-13. Distribution of Soil Depths from Surface Field Measurements for Soil Depth Class 4 (Shallow Soils) 
These data have been subjected to a "W test" (Gilbert 1987 [DIRS 163705], pp. 158 to 162; Tables A6, A7, and A8). The test results support the hypothesis that the data are lognormally distributed at the 0.02 significance level, but not at the 0.05 significance level. The test produced $\mathrm{W}=0.925$. Values greater than 0.920 are required to accept a lognormal distribution hypothesis at the 0.02 level, and values greater than 0.934 are required to accept the hypothesis at the 0.05 level. A similar test for the alternate hypothesis, that the data are normally distributed, shows that this hypothesis should be rejected. This test produced $\mathrm{W}=0.49$. Values greater than 0.92 are required to accept the normal distribution hypothesis. The fit to a lognormal distribution is illustrated on Figure 6-14 as a probability plot. This result and the general observation that depths in this class should be non-zero, because the soil mapping indicates that some cover was present but that soil depths generally fall at the shallow end of the range, result in the selection of a lognormal distribution as the preferred option for describing depth in Soil Depth Class 4. The calculated lognormal distribution (Section B.1.8) from field exposure measurements in DTN: GS011208312212.004 [DIRS 176317], Table S02086_001, has a $\log _{e}$ transformed mean of $-1.29 \mathrm{~m}(0.27 \mathrm{~m})$ and a $\log _{e}$ transformed standard deviation of $0.88 \mathrm{~m}$.

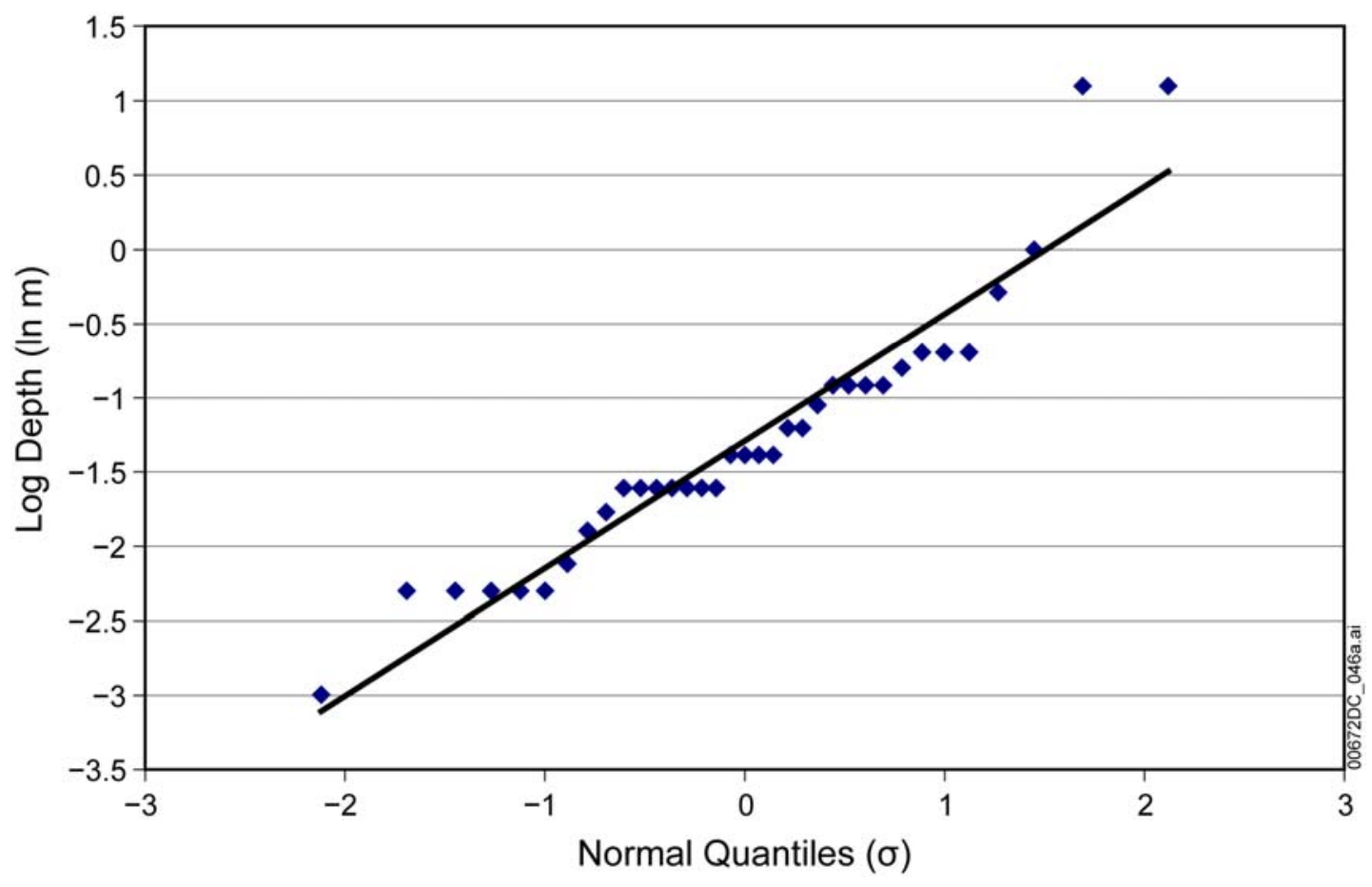

Sources: Table 6-6; Appendix B, Section B.1.8 and Taylor soil depth.xls, worksheet 'Wtest'.

Figure 6-14. Probability Plot of the $\log _{e}$ Transformed Data Shown in Figure 6-13

Borehole data (Table A-1) are also available for Soil Depth Class 4. These data were not used, however, in the evaluation of this class, because of significant uncertainties in any soil depths reported for boreholes located on shallow soils. These uncertainties result from borehole and drill pad construction practices and environmental permit requirements that were in effect when the holes were drilled. Soil Depth Class 4 consists of upland areas that can have steep slopes. The construction of drill pads in these areas requires considerable cutting and filling that will remove the native soil before drilling begins. Drill pad construction records for the following 
boreholes in this group also indicate that soil were present and removed prior to drilling, because of environmental permit stipulations on all land-disturbing activities that required the removal and storage of the top 0.30 to $0.35 \mathrm{~m}$ of topsoil at the start of construction for use in post-activity site reclamation:

- USW SD-7: "Stripped \& stacked topsoil" on first day of drill pad construction (YMP 1994 [DIRS 176589], RIS p. 1) followed by considerable cutting and filling for the drill pad (YMP 1994 [DIRS 176590], RIS p. 16)

- USW UZ-7A: "Site preparation will include topsoil removal and storage, excavation of rock material, and installation of fill material...Topsoil shall be salvaged down to 14 inches or until bedrock is reached" (YMP 1994 [DIRS 176591], RIS pp. 4 and 28)

- UE-25 NRG\#4: "Topsoil, down to bedrock or 30 centimeters, whichever is shallowest, shall be salvaged wherever topsoil disturbance (i.e., movement) will occur...Salvaged topsoil at the drill pad shall be stored adjacent to the drill pad at a location opposite the access road" (YMP 1993 [DIRS 176592], RIS p. 23).

In addition, the inspection of four of the drillpads in this group, those being UE-25 NRG\#3, UE25 NRG\#5, USW WT-24, and USW SD-9, show that these drillpads are cuts. The soils surrounding these cuts have average depths of about $0.7 \mathrm{~m}$ (Section 6.1.2) (Sanchez 2006 [DIRS 176569], pp. 48 to 67), while borehole logs show a zero depth. These factors make the use of borehole data in these areas suspect.

The distribution of intermediate depth soils that are assigned to Soil Depth Class 3 is based on the depth range from soil mapping of $0.5 \mathrm{~m}$ to greater than $3 \mathrm{~m}$ (Table 6-5), and on qualified data from field measurements and for boreholes located in cells assigned to this class. Field measurements represent locations of natural and artificial exposures measured at the surface where soil depth was recorded. Because there were only five field measurements, the field measurements and borehole data were combined for the analysis of this class. The field measurements with "Deposit Unit" designations of "coll" (DTN: GS011208312212.004 [DIRS 176317], Table S02086_001) were interpreted to correspond to Soil Depth Class 3. These measurements range between 2 and $3 \mathrm{~m}$. Borehole location data (Table A-1) were used in conjunction with the soil depth class assignments from output DTN: MO0606SPASDFIM.005 (Figure 6-12) to determine which boreholes are located in Soil Depth Class 3.

Qualified boreholes belonging to Soil Depth Class 3 with their associated soil depth are listed in Table 6-7, along with the data from the field measurements. Depths from qualified boreholes, shown in Figure 6-15, range from 0 to $20 \mathrm{~m}$ with all but one borehole in the 0 to $6 \mathrm{~m}$ range.

A significant number of boreholes show a zero depth for the soils. As discussed, for Soil Depth Class 4, these values are interpreted to be the result of drill pad construction operations and environmental requirements for removing and stockpiling soil in disturbed areas. Soil mapping shows that these locations are in areas of footslope colluvium and alluvium that should be thicker and more continuous than Soil Depth Class 4. Therefore, for the purposes of calculating the distribution, zero values were assigned a value of $0.5 \mathrm{~m}$ to correspond with the minimum value shown for the range estimated from soil mapping (Table 6-5). 
Data Analysis for Infiltration Modeling: Technical Evaluation of Previous Soil Depth Estimation Methods and Development of Alternate Parameter Values

Table 6-7. Field Measurements and Qualified Borehole Depths Used to Calculate Depth Distributions and Unqualified Borehole Depths Used for Corroboration for Soil Depth Class 3

\begin{tabular}{|c|c|c|}
\hline Borehole ID & Soil Depth (m) & LN Depth (m) \\
\hline \multicolumn{3}{|c|}{ Qualified Data } \\
\hline USW UZ-N32 & 0.00 & -0.69315 \\
\hline UE-25 RF\#28 & 3.05 & 1.11449 \\
\hline UE-25 RF\#15 & 0.00 & -0.69315 \\
\hline UE-25 RF\#23 & 19.51 & 2.97078 \\
\hline UE-25 NRG\#2 & 0.00 & -0.69315 \\
\hline USW NRG-6 & 0.00 & -0.69315 \\
\hline USW NRG-7a & 5.18 & 1.64511 \\
\hline USW UZ-N33 & 3.99 & 1.38451 \\
\hline UE-25 NRG\#2a & 2.07 & 0.72882 \\
\hline UE-25 NRG\#2b & 0.67 & -0.40048 \\
\hline Field Measurement & 2.00 & 0.69315 \\
\hline Field Measurement & 3.00 & 1.09861 \\
\hline Field Measurement & 2.50 & 0.91629 \\
\hline Field Measurement & 2.00 & 0.69315 \\
\hline Field Measurement & 3.00 & 1.09861 \\
\hline \multicolumn{3}{|c|}{ Sample Statistics for Qualified Data } \\
\hline LN Mean $(\mathrm{m})$ & & 0.61 \\
\hline LN Standard Deviation (m) & & 1.07 \\
\hline Median (m) (not log transformed) & & 2.07 \\
\hline \multicolumn{3}{|c|}{$\begin{array}{l}\text { Unqualified Data } \\
\end{array}$} \\
\hline USW NRG-7 & 0.00 & NA \\
\hline USW UZ-N80 & 0.00 & NA \\
\hline USW UZ-N25 & 0.00 & NA \\
\hline USW UZ-N26 & 0.00 & NA \\
\hline UE-25 UZN\#23 & 0.00 & NA \\
\hline UE-25 UZN\#30 & 0.38 & NA \\
\hline
\end{tabular}

Sources: Table 4-1; Table A-1; DTN: GS011208312212.004 [DIRS 176317], Table S02086_001; Appendix B, Section B.1.6 for Excel® calculations.

NOTE: $\quad$ Depths of $0.00 \mathrm{~m}$ changed to $0.5 \mathrm{~m}$ to calculate LN depth. LN depths were not calculated for the unqualified data.

$\mathrm{LN}=$ natural logarithm; NA = not applicable. 
Data Analysis for Infiltration Modeling: Technical Evaluation of Previous Soil Depth Estimation Methods and Development of Alternate Parameter Values
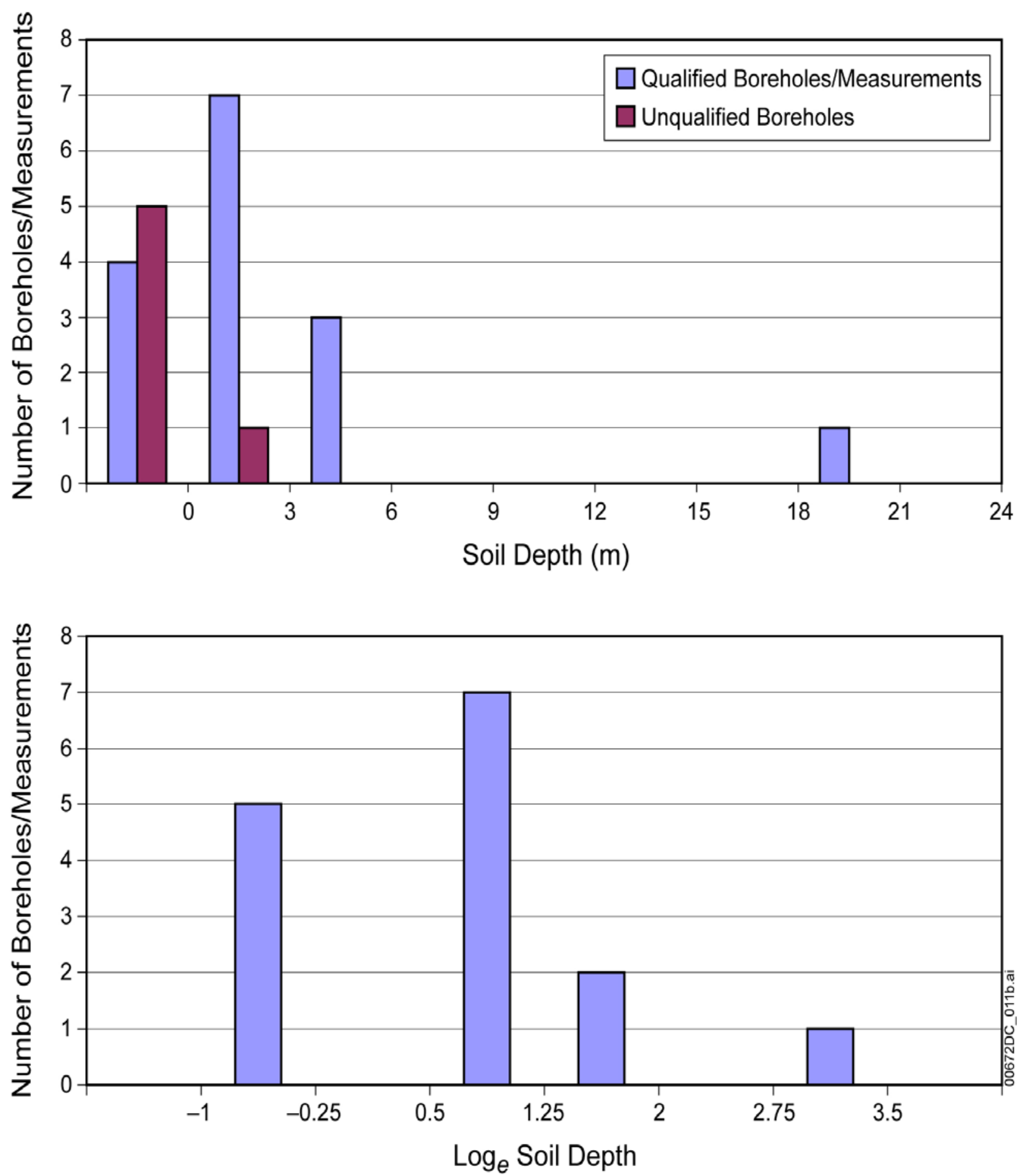

Sources: Table 6-7; Appendix B, Section B.1.6 and Final calcs. for report.xls, worksheet 'Class 3'. NOTE: Soil Depth $(\mathrm{m})$ shows linear depths, while $\log _{\mathrm{e}}$ Soil Depth shows log transformed measurements.

Figure 6-15. Distribution of Soil Depths from Boreholes and Surface Field Measurements for Soil Depth Class 3 (Intermediate Depth Soils) 
A lognormal distribution was selected to represent depth distribution for Soil Depth Class 3. The lognormal hypothesis is shown by a W test (Section B.1.10) to be accepted at the 0.05 significance level for the qualified data (Table 6-7). This W test, however, is based on a small number of data points and the uncertainty in the depth values for the qualified boreholes in the class must be considered. A probability plot for these data is illustrated on Figure 6-16. The selection of a lognormal distribution is also based on the description of the soil unit in this class, which indicates that soil is gradational into the soils of Soil Depth Class 4 and are generally thick enough to obscure underlying bedrock (DTNs: GS940108315142.004 [DIRS 160344], ACC: MOL.20021001.0238; GS940108315142.005 [DIRS 160345], ACC: MOL.20021203.0409; GS940708315142.008 [DIRS 160346], ACC: MOL.20021001.0261; and GS950408315142.004 [DIRS 160347], ACC: MOL.20020827.0355). These descriptions, and the indication from borehole data that the majority of points should fall in the shallow end of the range, led to selecting a distribution that would skew the depths toward the shallow end the range. The calculated values describing the distribution are listed in Table 6-7. The distribution is considered reasonable because it preserves the bias toward the low end of the range indicated by borehole data and it generally corresponds to the range derived from surface mapping. Qualified borehole values are also corroborated by unqualified borehole depth values (Figure 6-15).

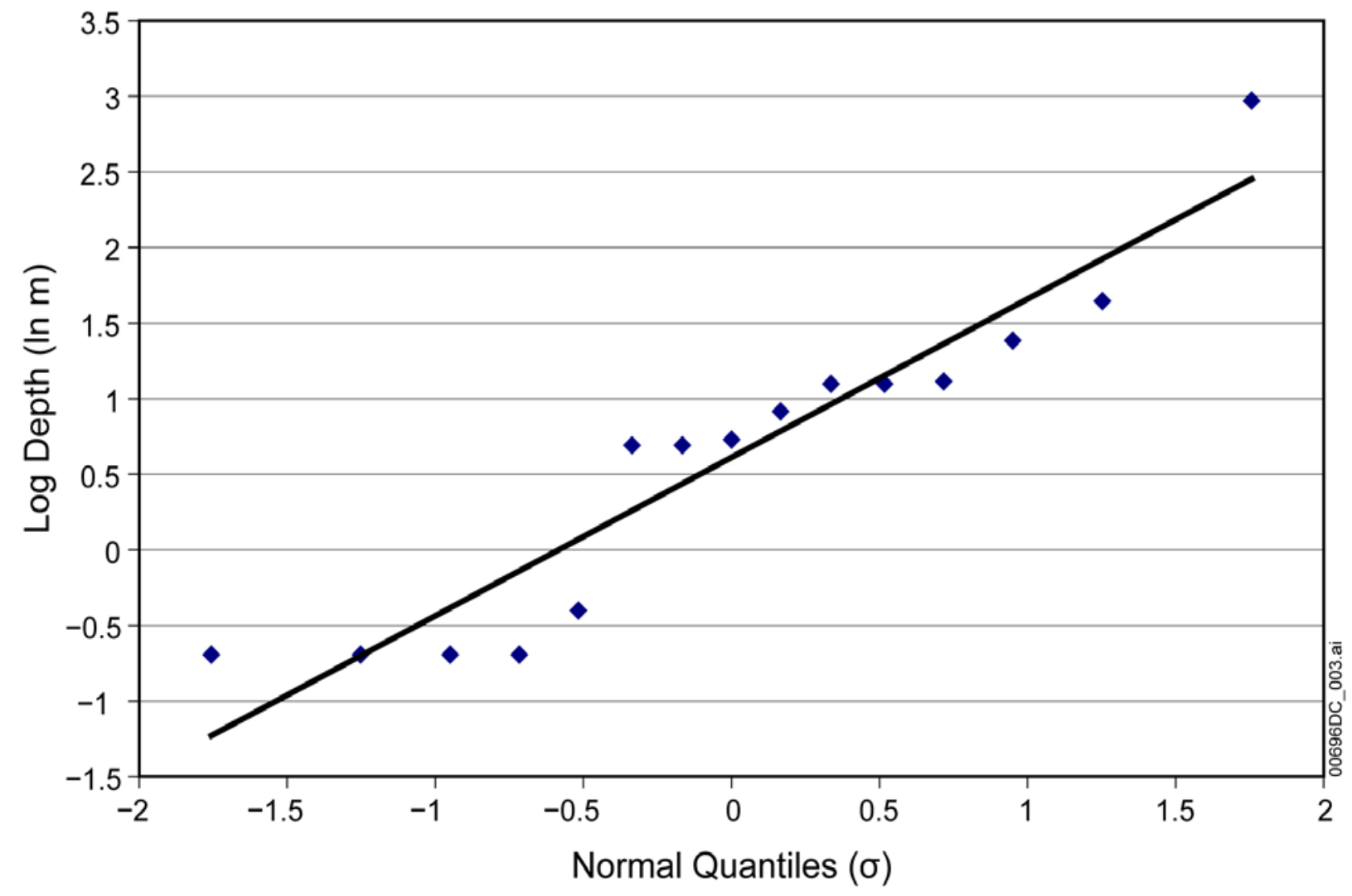

Sources: Table 6-7; Appendix B, Section B.1.10 and Final calcs. for report.xls, worksheet 'W-testC3'.

Figure 6-16. Probability Plot of the Data Listed in Table 6-7 
The distribution of moderately deep soils, assigned to Soil Depth Class 2, is based on qualified data for boreholes located in cells assigned to this class. Borehole location data (Table A-1) were used in conjunction with soil depth class assignments (Figure 6-12) from output DTN: MO0606SPASDFIM.005 to determine which boreholes are located in Soil Depth Class 2. Qualified boreholes in Soil Depth Class 2 with their associated soil depth are listed in Table 6-8. Borehole soil depths in this class, shown in Figure 6-17, range from 0 to $65 \mathrm{~m}$. This class includes the greatest number of borehole data points. The distribution of data (Figure 6-17) indicates that a normal distribution is most appropriate for characterizing soil depths in this class. The selection of a normal distribution was tested using D'Agostino's Test (Gilbert 1987 [DIRS 163705]), which supports the hypothesis that data are normally distributed at the 0.02 significance level, but not at the 0.05 significance level. As illustrated by the probability plot in Figure 6-18, this lower degree of confidence is the result of the truncation of the distribution at the zero value (Figure 6-17) and the number of points with the same value of zero. The parameters for a normal distribution based on qualified borehole data are listed in Table 6-8.

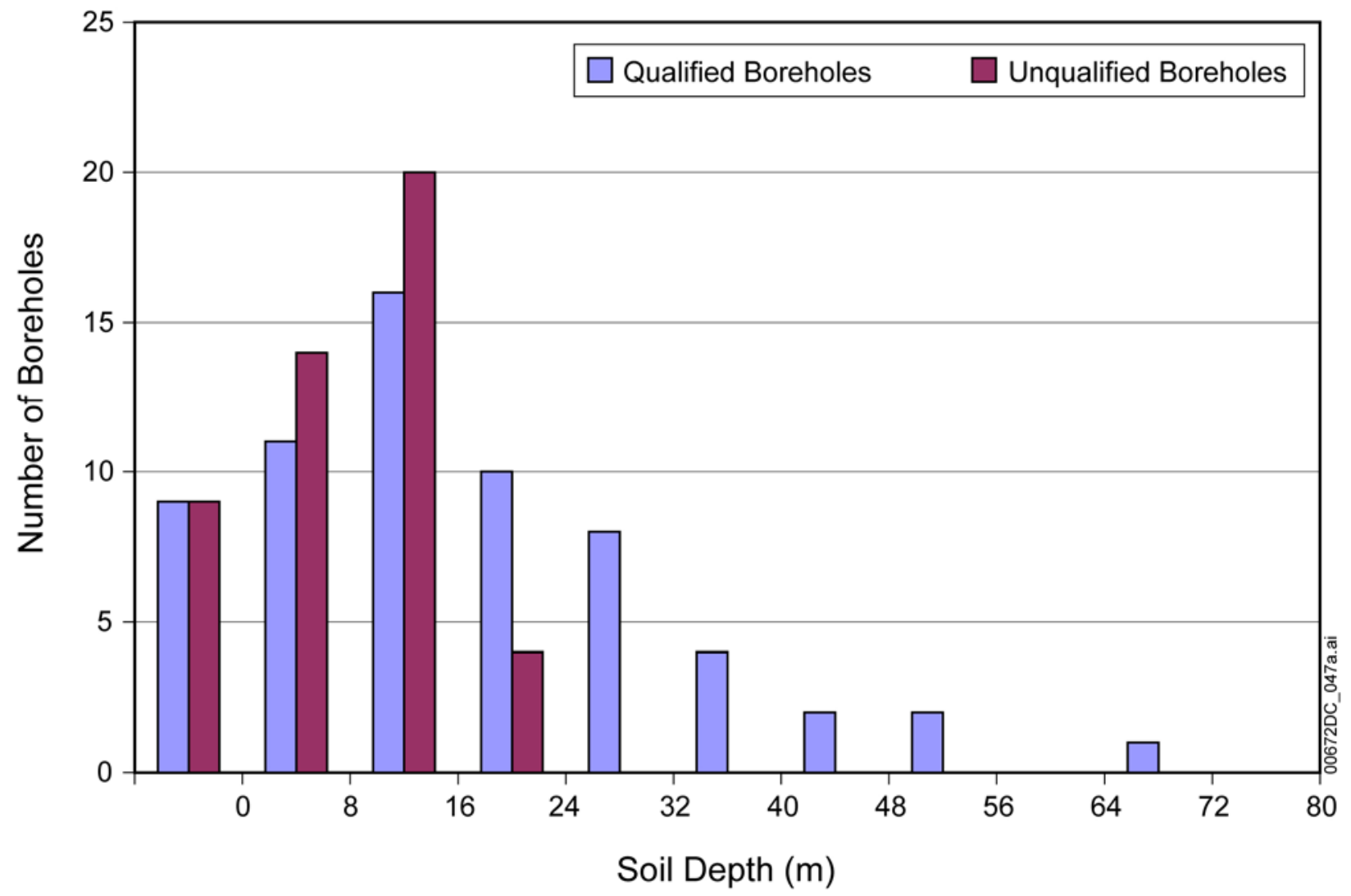

Source: Appendix B, Section B.1.6 and Final calcs. for report.xls, worksheet 'Class 2'.

NOTES: Qualified and unqualified data are listed in Table 6-8

Figure 6-17. Distribution of Soil Depths in Qualified and Unqualified Boreholes for Soil Depth Class 2 (Moderately Deep Soils) 
Data Analysis for Infiltration Modeling: Technical Evaluation of Previous Soil Depth Estimation Methods and Development of Alternate Parameter Values

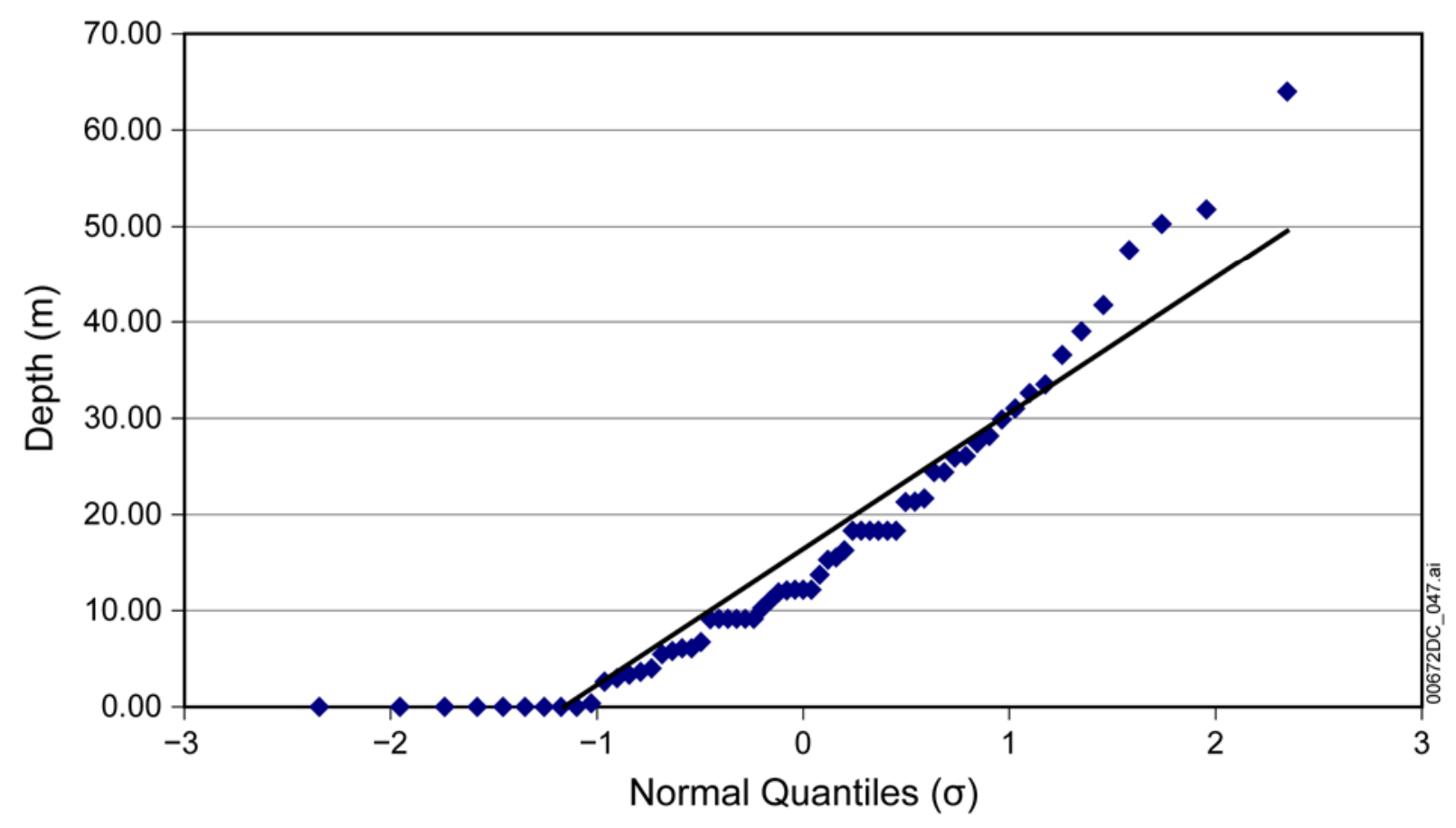

Source: Appendix B, Section B.1.11 and Final calcs. for report.xls, worksheet 'DAgtest'.

Figure 6-18. Probability Plot of the Qualified Data Shown in Figure 6-17

Table 6-8. Qualified Borehole Depths Used to Calculate Depth Distribution and Unqualified Borehole Depths Used for Corroboration for Soil Depth Class 2

\begin{tabular}{|l|c|}
\hline \multicolumn{1}{|c|}{ Borehole Identification } & Soil Depth $(\mathbf{m})$ \\
\hline \multicolumn{1}{|c|}{ Qualified Data } \\
\hline UE-25 RF\#13 & 26.06 \\
\hline UE-25 RF\#22 & 24.38 \\
\hline UE-25 UZ\#4 & 11.89 \\
\hline UE-25 RF\#18 & 18.29 \\
\hline UE-25 RF\#14 & 31.03 \\
\hline UE-25 RF\#19 & 36.58 \\
\hline UE-25 RF\#29 & 25.91 \\
\hline UE-25 RF\#17 & 28.16 \\
\hline USW G-1 & 18.29 \\
\hline USW G-2 & 0.00 \\
\hline USW H-1 & 0.00 \\
\hline UE-25p\#1 & 39.01 \\
\hline USW G-4 & 9.14 \\
\hline USW a\#1 & 9.14 \\
\hline UE-25 a\#4 & 9.14 \\
\hline UE-25 a\#5 & 27.43 \\
\hline UE-25 a\#6 & 6.10 \\
\hline UE-25 a\#7 & 50.29 \\
\hline UE-25 b\#1 & 47.55 \\
\hline
\end{tabular}


Data Analysis for Infiltration Modeling: Technical Evaluation of Previous Soil Depth Estimation Methods and Development of Alternate Parameter Values

Table 6-8. Qualified Borehole Depths Used to Calculate Depth Distribution and Unqualified Borehole Depths Used for Corroboration for Soil Depth Class 2 (Continued)

\begin{tabular}{|c|c|}
\hline Borehole Identification & Soil Depth $(m)$ \\
\hline \multicolumn{2}{|c|}{ Qualified Data (Continued) } \\
\hline UE-25 c\#1 & 0.00 \\
\hline UE-25 c\#2 & 21.31 \\
\hline UE-25 c\#3 & 24.41 \\
\hline USW H-3 & 0.00 \\
\hline USW H-4 & 0.00 \\
\hline USW H-5 & 0.00 \\
\hline USW H-6 & 9.11 \\
\hline USW UZ-1 & 12.19 \\
\hline USW UZ-6 & 0.00 \\
\hline USW WT-1 & 9.14 \\
\hline USW WT-2 & 18.29 \\
\hline UE-25 WT\#3 & 3.35 \\
\hline UE-25 WT\#4 & 15.54 \\
\hline UE-25 WT\#6 & 51.82 \\
\hline USW WT-7 & 12.19 \\
\hline USW WT-10 & 18.29 \\
\hline USW WT-11 & 12.19 \\
\hline UE-25 WT\#12 & 18.29 \\
\hline UE-25 WT\#14 & 32.61 \\
\hline UE-25 WT\#15 & 64.01 \\
\hline UE-25 WT\#16 & 41.76 \\
\hline UE-25 WT\#17 & 9.14 \\
\hline UE-25 WT\#18 & 0.00 \\
\hline UE-25 RF\#26 & 21.64 \\
\hline UE-25 RF\#16 & 16.25 \\
\hline NC-EWDP-18P & 13.72 \\
\hline UE-25 RF\#20 & 21.34 \\
\hline UE-25 RF\#24 & 6.10 \\
\hline UE-25 RF\#25 & 18.29 \\
\hline UE-25 RF\#21 & 33.53 \\
\hline USW UZ-N57 & 0.37 \\
\hline USW UZ-N58 & 6.74 \\
\hline USW UZ-N59 & 3.99 \\
\hline USW UZ-N61 & 2.99 \\
\hline USW UZ-N54 & 5.79 \\
\hline USW UZ-N35 & 3.63 \\
\hline USW UZ-N37 & 11.06 \\
\hline USW UZN\#63 & 2.62 \\
\hline USW UZ-N34 & 15.27 \\
\hline USW UZ-N31 & 0.00 \\
\hline UE-25 UZ\#16 & 10.27 \\
\hline UE-25 ONC \#1 & 29.87 \\
\hline
\end{tabular}


Data Analysis for Infiltration Modeling: Technical Evaluation of Previous Soil Depth Estimation Methods and Development of Alternate Parameter Values

Table 6-8. Qualified Borehole Depths Used to Calculate Depth Distribution and Unqualified Borehole Depths Used for Corroboration for Soil Depth Class 2 (Continued)

\begin{tabular}{|c|c|}
\hline Borehole Identification & Soil Depth (m) \\
\hline \multicolumn{2}{|c|}{ Qualified Data (Continued) } \\
\hline USW UZ-N38 & 5.46 \\
\hline USW UZ-14 & 12.10 \\
\hline \multicolumn{2}{|c|}{ Sample Statistics for Qualified Data (lower bound set at $0.5 \mathrm{~m}$ ) } \\
\hline Mean & 16.47 \\
\hline Standard Deviation & 14.61 \\
\hline Median & 12.19 \\
\hline \multicolumn{2}{|c|}{ Unqualified Data } \\
\hline USW SD-12 & 0.00 \\
\hline USW UZ-N40 & 0.88 \\
\hline USW UZ-N41 & 4.88 \\
\hline USW UZ-N42 & 0.00 \\
\hline USW UZ-N43 & 8.23 \\
\hline USW UZ-N45 & 10.67 \\
\hline USW UZ-N46 & 0.00 \\
\hline USW UZ-N47 & 10.06 \\
\hline USW UZ-N48 & 0.00 \\
\hline USW UZ-N50 & 2.74 \\
\hline USW UZ-N51 & 4.27 \\
\hline USW UZ-N52 & 2.13 \\
\hline USW UZ-N67 & 5.79 \\
\hline USW UZ-N68 & 15.24 \\
\hline USW UZ-N69 & 8.23 \\
\hline USW UZ-N77 & 11.58 \\
\hline USW UZ-N82 & 6.70 \\
\hline USW UZ-N83 & 0.00 \\
\hline USW UZ-N84 & 6.09 \\
\hline USW UZ-N86 & 0.00 \\
\hline USW UZ-N87 & 5.79 \\
\hline USW UZ-N89 & 10.06 \\
\hline USW UZ-N90 & 9.91 \\
\hline USW UZ-N93 & 0.00 \\
\hline USW UZ-N94 & 0.00 \\
\hline USW UZ-N95 & 0.00 \\
\hline USW UZ-N98 & 0.30 \\
\hline USW UZ-N24 & 0.15 \\
\hline UE-25 UZN \#1 & 8.32 \\
\hline UE-25 UZN \#3 & 2.74 \\
\hline UE-25 UZN \#4 & 7.47 \\
\hline UE-25 UZN \#5 & 13.56 \\
\hline UE-25 UZN \#6 & 12.04 \\
\hline UE-25 UZN \#7 & 12.34 \\
\hline UE-25 UZN \#8 & 12.19 \\
\hline
\end{tabular}


Data Analysis for Infiltration Modeling: Technical Evaluation of Previous Soil Depth Estimation Methods and Development of Alternate Parameter Values

Table 6-8. Qualified Borehole Depths Used to Calculate Depth Distribution and Unqualified Borehole Depths Used for Corroboration for Soil Depth Class 2 (Continued)

\begin{tabular}{|l|c|}
\hline \multicolumn{1}{|c|}{ Borehole Identification } & Soil Depth $(\mathrm{m})$ \\
\hline \multicolumn{1}{|c|}{ Unqualified Data (Continued) } \\
\hline UE-25 UZN \#9 & 10.67 \\
\hline UE-25 UZN \#12 & 13.72 \\
\hline UE-25 UZN \#13 & 13.41 \\
\hline UE-25 UZN \#14 & 13.41 \\
\hline UE-25 UZN \#18 & 17.07 \\
\hline UE-25 UZN \#20 & 11.89 \\
\hline UE-25 UZN \#21 & 8.38 \\
\hline UE-25 UZN \#22 & 5.94 \\
\hline UE-25 UZN \#56 & 17.07 \\
\hline UE-25 UZN \#60 & 8.08 \\
\hline UE-25 UZN \#97 & 17.68 \\
\hline UE-29 UZN \#92 & 17.68 \\
\hline
\end{tabular}

Sources: Table 4-1; Table A-1; Appendix B, Section B.1.6 for Excel $\mathbb{R}$ calculations.

This distribution should be implemented as a left-truncated normal distribution with a point of truncation at $0.5 \mathrm{~m}$ because negative values are not possible and a spike at the zero value would be undesirable because this class is mapped as alluvial covered valley floors and should not have bedrock exposures. As was seen with the shallower soil depth classes, a few borehole logs indicate zero soil depth. These boreholes are considered to have been affected by drill pad construction practices because the soil mapping indicate the presence of alluvial cover. As illustrated on Figure 6-9, these boreholes are near the contact with shallower classes and at the base of slopes cuts may have been required to create a drill pad. A truncation point of $0.5 \mathrm{~m}$ was selected because the apparent ability of drill pad excavation to remove all soil indicates that some depths in this class must relatively shallow. In addition, as shown on Figure 6-12, Soil Depth Class 2 may be in contact with either Soil Depth Class 3 or Soil Depth Class 4. Therefore, the depth at the contact could be as small as 0.1 to $0.5 \mathrm{~m}$, depending on the class with which Soil Depth Class 2 is in contact (Table 6-5), and, thus, a minimum value of $0.5 \mathrm{~m}$ is reasonable for this class. The distribution is considered reasonable because it closely corresponds to the qualified borehole data. The distribution is also corroborated by the unqualified data from additional boreholes (Figure 6-17). Physically, the class represents shallower alluvial deposits extending outward for $300 \mathrm{~m}$ from the contact with thin colluvial deposits. Because buried topography can exist at the alluvial-bedrock contact, the increase in depth is not necessarily uniform so that the random depth pattern created by sampling from the distribution is not necessarily at odds with reality. A distribution of this type that ranges from near zero meters to depths of about $40 \mathrm{~m}$ should be an adequate representation of depths in this area (Figure 6-11).

Because of the sparse amount of qualified borehole data for very deep soils, the distribution recommended for Soil Depth Class 1 is based on the alluvial depth range given in output DTN: MO0012MWDGFM02.002 [DIRS 153777], as illustrated in the GFM (BSC 2004 [DIRS 170029], Figure 6-10). The distribution is also based on an extension of the range of depths given by borehole data for Soil Depth Class 2 (Figure 6-17). The qualified data for 
boreholes located in cells assigned to this class were also used to support the evaluation. Borehole location data (Table A-1) were used in conjunction with soil depth class assignments (Figure 6-12) in output DTN: MO0606SPASDFIM.005 to determine which boreholes are located in Soil Depth Class 1. Qualified and unqualified boreholes in Soil Depth Class 1, with their associated soil depth, are listed in Table 6-9. There are only two qualified boreholes located within this class. The number of unqualified data points is also small. Qualified boreholes (Table 6-9) give a range of soil depths from 67 to $132 \mathrm{~m}$. Unqualified points range from 148 to $157 \mathrm{~m}$ in soil depth. The distribution of qualified and unqualified data points is shown on Figure 6-19. Due to the small number of data values, the lower bound for Class 1 is set $40 \mathrm{~m}$, which corresponds approximately with the upper bound depth of Soil Depth Class 2 .

Table 6-9. Qualified Borehole Soil Depths Used to Determine the Depth Distribution Limits and Unqualified Borehole Soil Depths for Corroboration for Soil Depth Class 1

\begin{tabular}{|l|c|l|}
\hline \multicolumn{1}{|c|}{ Borehole ID } & Soil Depth $(\mathbf{m})$ & \multicolumn{1}{c|}{ Qualification Status } \\
\hline UE-25 J\#13 & 132.59 & Qualified - PVAR \\
\hline UE-25 WT\#13 & 67.06 & Qualified - PVAR \\
\hline UE-25 J\#12 & 156.97 & Unqualified \\
\hline UE-25 JF\#3 & 148.44 & Unqualified \\
\hline
\end{tabular}

Sources: Table 4-1; Table A-1; Appendix B, Section B.1.6 for Excel® calculations.

ID = identification; PVAR = Process Validation and Reengineering.

Data from the GFM (BSC 2004 [DIRS 170029], Figure 6-10) were compared to the outline of the very deep soil depth class (Figure 6-12). The correspondence between the depth contours from the GFM (BSC 2004 [DIRS 170029]; DTN: MO0012MWDGFM02.002 [DIRS 153777]) and the contact between Soil Depth Classes 1 and 2 is reasonable but not exact. The GFM indicates a depth ranging between approximately 25 and $60 \mathrm{~m}$ for this contact. The maximum depth indicated by the GFM for this class is somewhat greater than $150 \mathrm{~m}$ in the southeast corner of the GFM area. Because Soil Depth Classes 1 and 2 are subdivisions of what is an essentially steadily increasing linear trend (Figure 6-11), the boundary between the two classes should agree or overlap slightly. Based on this information, a simple uniform distribution ranging from 40 to $150 \mathrm{~m}$ is recommended for modeling purposes.

The minimum value generally corresponds to the upper bound of the range defined by Soil Depth Class 2 (Figure 6-17) with some slight overlap of the outlying points and falls within the range shown by the GFM (BSC 2004 [DIRS 170029], Figure 6-10). The maximum value represents a rounded value based on the GFM (BSC 2004 [DIRS 170029]; DTN: MO0012MWDGFM02.002 [DIRS 153777]). The depths for the qualified and unqualified boreholes also fall within this range (Figure 6-19). This simplified approach of using a uniform distribution is reasonable because depths in this class are significantly below, by greater than $30 \mathrm{~m}$, the depths where infiltration processes were considered by previous infiltration models to allow water to reach the soil-bedrock contact (BSC 2004 [DIRS 170007], Section 6.1.2). Therefore, a more detailed evaluation of soil depths in this class is not required at this time. This conclusion, however, is subject to confirmation by future modeling efforts. If a more detailed prediction of depth for the cells in Soil Depth Class 1 is required, DTN: MO0012MWDGFM02.002 [DIRS 153777] can be used to generate values, as were generated for the GFM (BSC 2004 [DIRS 170029], Figure 6-10). 


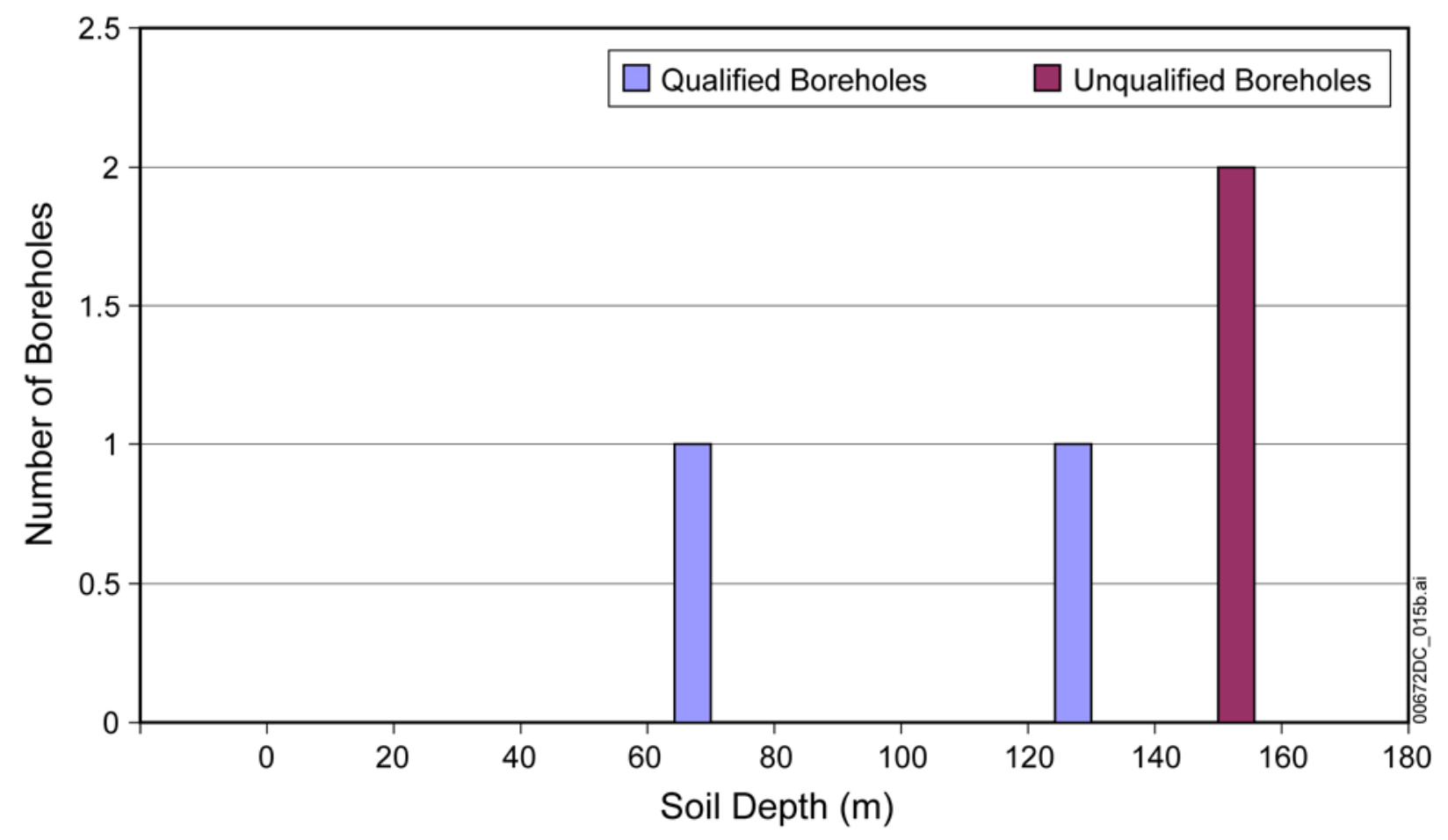

Sources: Table 6-9; Appendix B, Section B.1.6 and Final calcs. for report.xls, worksheet 'Class 1'.

Figure 6-19. Distribution of Soil Depths in Qualified and Unqualified Boreholes for Soil Depth Class 1 (Very Deep Soils)

\subsubsection{Uncertainty in Estimating Soil Depth Parameters for Modeling}

The results discussed in this analysis are subject to several types of uncertainty. One type of uncertainty pertains to the natural variability in soil depth that occurs at all scales in the area. This uncertainty is most visible in shallow soil classes where depths can vary considerably within the area defined by a $30 \times 30 \mathrm{~m}$ grid cell, in addition to the variability in average depths that can occur between grid cells. Although the sample distributions listed in Table 6-10 are intended to be used to estimate the variability in average depth that occurs between grid cells for modeling purposes, they are actually based on point values made at specific locations within a cell. The variability of these point measurements also demonstrate the variability that can occur within an individual grid cell. This local variability is corroborated by trench logs in the area that can provide an illustration of the variability in depth along a continuous exposure. For example, DTN: GS980183117462.001 [DIRS 176939], ACC: MOL.19990706.0190, shows depths ranging from 0.8 to $2.2 \mathrm{~m}$ over a distance of $11 \mathrm{~m}$ in Soil Depth Class 4 to the east of the Solitario Canyon fault in Trench SCF-T1.

A second type of uncertainty pertains to measurement errors made when determining soil depths at sampling localities. Soil Depth Classes 3 and 4 use data from surface field measurements at natural exposures, such as gullies and stream cuts, as well as from manmade exposures, such as cuts for drill pads and trenches. These soil-depth measurements were made with a tape measure and measurement uncertainty of these values is small. The principal uncertainty in these measurements is the accuracy in defining the soil-bedrock contact at the field exposure. 
Data Analysis for Infiltration Modeling: Technical Evaluation of Previous Soil Depth Estimation Methods and Development of Alternate Parameter Values

Table 6-10. Summary of Recommended Distributions for Soil Depth Classes Used in Alternate Evaluation

\begin{tabular}{|c|c|c|c|c|c|}
\hline Soil Depth Class Designator & 1 & 2 & 3 & 4 & 5 \\
\hline Soil Depth Class & $\begin{array}{l}\text { Very Deep } \\
\text { Alluvium }\end{array}$ & $\begin{array}{l}\text { Moderately Deep } \\
\text { Alluvium }\end{array}$ & $\begin{array}{l}\text { Intermediate } \\
\text { Depth Soils }\end{array}$ & $\begin{array}{l}\text { Shallow Soils- } \\
\text { Colluvium }\end{array}$ & $\begin{array}{l}\text { Exposed } \\
\text { Bedrock }\end{array}$ \\
\hline Sample Distribution Type & Uniform & $\begin{array}{l}\text { Left-Truncated } \\
\text { Normal (truncated } \\
\text { at } 0.5 \mathrm{~m} \text { ) }\end{array}$ & Lognormal & Lognormal & $\begin{array}{l}\text { Single } \\
\text { Value }\end{array}$ \\
\hline Sample Mean & NA & $16.47(\mathrm{~m})$ & $\begin{array}{l}0.61(\mathrm{LN} \mathrm{m}) \\
(1.84 \mathrm{~m})\end{array}$ & $\begin{array}{l}-1.29(\mathrm{LN} \mathrm{m}) \\
(0.27 \mathrm{~m})\end{array}$ & NA \\
\hline Sample Standard Deviation & NA & $14.61(\mathrm{~m})$ & $1.07(\mathrm{LN} \mathrm{m})$ & $0.88(\mathrm{LN} \mathrm{m})$ & NA \\
\hline $\begin{array}{l}\text { Sample Median (also } \\
\text { Estimated Population Median) }\end{array}$ & $95 \mathrm{~m}$ & $12.19(\mathrm{~m})$ & $2.07(\mathrm{~m})$ & $0.25(\mathrm{~m})$ & NA \\
\hline Sample Minimum Value $(\mathrm{m})$ & $40(\mathrm{~m})$ & 0.5 & NA & NA & 0 \\
\hline Sample Maximum Value (m) & $150(\mathrm{~m})$ & NA & NA & NA & 0 \\
\hline Estimated Population Mean & $95(\mathrm{~m})$ & $16.47(\mathrm{~m})$ & $3.25(\mathrm{~m})$ & $0.40(\mathrm{~m})$ & 0 \\
\hline $\begin{array}{l}\text { Confidence Interval for } \\
\text { Population Mean at } 80 \% \text { Limit }\end{array}$ & - & $\begin{array}{l}14.09 \text { to } \\
18.86(\mathrm{~m})\end{array}$ & $\begin{array}{c}2.21 \text { to } \\
5.73(\mathrm{~m})\end{array}$ & $\begin{array}{c}0.33 \text { to } \\
0.52(\mathrm{~m})\end{array}$ & NA \\
\hline $\begin{array}{l}\text { Confidence Interval for } \\
\text { Population Mean at } 90 \% \text { Limit }\end{array}$ & - & $\begin{array}{c}13.40 \text { to } \\
19.54(\mathrm{~m})\end{array}$ & $\begin{array}{l}2.00 \text { to } \\
7.11(\mathrm{~m}) \\
\end{array}$ & $\begin{array}{c}0.31 \text { to } \\
0.57(\mathrm{~m}) \\
\end{array}$ & NA \\
\hline $\begin{array}{l}\text { Confidence Interval for } \\
\text { Population Median at } 80 \% \\
\text { Limit }\end{array}$ & NA & NA & $\begin{array}{c}0.44 \text { to } \\
7.73(\mathrm{~m}) \\
\end{array}$ & $\begin{array}{c}0.09 \text { to } \\
0.86(\mathrm{~m})\end{array}$ & NA \\
\hline $\begin{array}{l}\text { Confidence Interval for } \\
\text { Population Median at } 90 \% \\
\text { Limit }\end{array}$ & NA & NA & $\begin{array}{c}0.28 \text { to } \\
12.05(\mathrm{~m})\end{array}$ & $\begin{array}{c}0.06 \text { to } \\
1.21(\mathrm{~m})\end{array}$ & NA \\
\hline
\end{tabular}

Sources: Output DTN: MO0606SPASDFIM.005; Appendix B, Final calcs. for report.xls, worksheets 'Class 2' and 'Class 3'; Appendix B, Taylor soil depth.xls, worksheet 'Wtest' (for Class 4).

$\mathrm{LN}=$ natural logarithm; NA = not applicable.

In contrast to the surface field measurements, the uncertainty in the borehole measurements used directly in Soil Depth Classes 1, 2, and 3 is more significant. This uncertainty is related to drill pad construction activities and to determining the alluvial-bedrock contact based on borehole information. Environmental stipulations generally require the removal of the uppermost 0.3 to $0.4 \mathrm{~m}$ of topsoil, which is then stored for use in site restoration. In addition, the creation of a level pad for a drill rig and support trailers can result in cuts that remove additional material. Therefore, borehole depth measurements can be low by amounts ranging from zero to three or more meters, because of drill pad construction operations (Sections 6.1.2 and 6.2.1). While this factor affects all boreholes, the most significant effect would occur in areas of shallow soil depth, because the amount of potentially removed soil during drill pad construction represents a large percentage of the total soil depth. To account for this factor in Soil Depth Classes 3 and 4, the zero soil depths have been modified to reflect the minimum values for each class as reported in soil mapping data. All depths determined from boreholes, however, probably represent minimum depths. Because of these concerns, borehole soil depths for Soil Depth Class 4 were not used. Countering this uncertainty factor is the uncertainty that a soil depth may have been increased by the addition of fill to create a level pad. Some borehole logs indicate considerable depths of artificial fill. Wherever fill was indicated in a log, the fill thickness was not included in the soil depth value. It is possible, however, that in all cases the fill was not differentiated from the soil-alluvium. 
Another area of uncertainty includes difficulty in determining the soil-bedrock contact in a borehole. For relatively shallow soils, the soil interval is generally augered or drilled, rather than cored, to set casing for subsequent coring operations in bedrock. Even if coring were to take place across the contact, the core recovery in the unconsolidated soils could be poor. Therefore, the contact depth for fill-soil or soil-bedrock contact could be an estimate by the geologist who logged the borehole and subjected the soil to some measure of uncertainty.

A third type of uncertainty is in the statistical estimation of population parameters using a relatively limited sample from the population. For Soil Depth Class 2, the uncertainty is quantified using a standard Student's $t$-distribution (Section B.1.12). The confidence interval for estimating the population mean from the sample values is given in Table 6-10.

Because Soil Depth Classes 3 and 4 are characterized as lognormal distributions, the sample mean is not the estimate of the population mean. The estimated population mean is calculated (Gilbert 1987 [DIRS 163705], Equation 13.7) as are the confidence limits for the population mean (Gilbert 1987 [DIRS 163705], Equations 13.13 and 13.14) (Section B.1.12). These values are shown in Table 6-10. This calculation indicates, for example, that the population mean for Soil Depth Class 4 is expected to be between 0.31 and $0.57 \mathrm{~m}$ with a $90 \%$ level of confidence. In a similar fashion, the population mean for Soil Depth Class 3 is expected to be between 2.0 and $7.11 \mathrm{~m}$ with a $90 \%$ level of confidence. Values would occur between 0.57 and $2.0 \mathrm{~m}$ for both classes because the distributions about these means overlap in this interval.

The calculated confidence intervals shown on Table 6-10 include the underlying assumptions that the population is normally (or lognormally) distributed and that the sample represents a random sample of the population. Tests for normality are discussed in Section 6.2.1. The field measurements of soil depth for Soil Depth Class 4 probably represent a reasonably random sample because the relatively steep terrain and thin soils create a large number of exposures randomly scattered across the area covered by this class. The field measurements for Soil Depth Class 3 may be more biased because, as the soils are thicker in this class, exposures may tend to preferentially occur in the thinner soils in the class rather than the thicker soils. Borehole locations were selected without regard to soil depth in Soil Depth Classes 3 and 4, but locations that were near roads and on level terrain were preferred when possible for cost and environmental reasons, which could introduce some bias into the sample. Boreholes in Soil Depth Classes 1 and 2 were mostly sited to study foundation conditions in the vicinity of proposed surface facilities in Midway Valley. Therefore, the borehole data points tend to be concentrated at this locality and are not randomly distributed across the area covered by these soil depth classes.

While uncertainties related to sample size can be quantified, the contribution of other types of uncertainty, to the total uncertainty in the estimate, are more difficult to quantify. Uncertainties for Soil Depth Class 4 are most likely to affect net infiltration because these soils are thin and more readily transmit water to the bedrock and because the majority of model cells above the repository block belong to this class. Uncertainties in this class are predominantly related to sample size, because borehole data with their attendant uncertainty were not used to evaluate this class. The principal uncertainty in this class is the natural variation in thickness in the shallow soils in this class. The infiltration model grid will assign a uniform soil depth value to a 
$30 \times 30 \mathrm{~m}$ grid cell. Actual conditions in the cell will likely vary between small areas of exposed bedrock and areas of soil cover with different depths.

Soil Depth Class 3 is subject to the greatest uncertainty because of a small sample size and because of the uncertainties related to borehole measurements in relatively shallow soils. This class, however, covers a small percentage of the model area (Figure 6-12). Modified values used for boreholes showing zero depth values compensate for some of this uncertainty. Most boreholes in this class underestimate soil depth by amounts of $0.3 \mathrm{~m}$ or more. Values for the confidence intervals around the estimated population mean (Table 6-10) include the range shown by surface field measurements that are not subject to this uncertainty. For this reason, and because underestimating the thickness would be a conservative bias, the confidence intervals on the population mean (Table 6-10) are considered the best estimate given the available data.

Recommendations herein result in distributions for soil depth classes that overlap. This is a departure from previous evaluations where ranges did not overlap. This is considered a reasonable approach that reflects the uncertainty, as shown by borehole data and observations from surficial deposits mapping, that results from the considerable natural variation in soil depth in the model area. A summary of recommended distributions representing the relevant qualified data for each soil depth class in this alternate evaluation is listed in Table 6-10 and is included in output DTN: MO0606SPASDFIM.005.

\subsubsection{Intended Use of Output and Limitations}

Intended as a possible basis for constructing input files for use in infiltration modeling are recommendations on the spatial distribution of soil depth classes, type of depth distribution to be used for each depth class, and estimates of the population mean with confidence intervals for the population mean, which are listed in Table 6-10 and included in output DTN: MO0606SPASDFIM.005, Summary of Recommended Distributions.doc. It is not intended that this analysis prescribe specific model input values or approaches to be used in addressing soil depth within a model. Modelers will need to consider the requirements of their model, the natural variation in soil thickness across the area, and any uncertainties associated with limited data sets in determining the approach that best meets their needs. Users of this analysis should also note that output DTN: MO0606SPASDFIM.005, revised soil depth file.csv and revised soil depth file.txt, describes the spatial extent of soil depth classes uses a grid specifically intended for use in an infiltration model. The choice of grid size and soil-depth-class definitions may not be completely suitable for other applications.

The proposed distributions are not based on a correlation between slope and soil depth as used in the infiltration model report (BSC 2004 [DIRS 170007]). While such a correlation may or may not exist, the existing data do not support such a correlation or provide data to quantify such a correlation if it exists. The limited data currently available are best characterized in a simple fashion as a distribution that can be used to characterize the cells in a particular soil depth class. This approach has the limitation of not being capable of predicting the depth value of an individual grid cell, as was done in the previous infiltration model report (BSC 2004 [DIRS 170007], p. 6-45) as a function of slope and depth class. The distribution can only be used to characterize the distribution of depths estimated to occur over a large area, but not the precise geometry of the depths within the area. 
There are many approaches that could be taken in implementing the recommended distributions listed in Table 6-10, which are provided for use given several considerations. In assigning depth values to use for modeling, two possible approaches are discussed. The first approach is to assign a value to each cell in a particular soil depth class by sampling, from the distribution for that soil depth class. This would result in the cells in the class having a range of values that follow the distribution and would require careful implementation, because the spatial relationship of soil depth from cell to cell must be taken into consideration. The depth of soil in each cell is not independent of the depth in the adjacent cell. Additionally, the relationship of soil depth cell to cell may also depend on whether the cells are oriented, generally, parallel or perpendicular to bedrock outcrops.

Parallel to outcrops, adjacent cells may have similar soil depths, but perpendicular to outcrops, soil depth may change significantly over a short distance. Therefore, an approach that assigns the depth to a cell, by random sampling from the probability distribution (Table 6-10), then repeats that random sampling for each of the remaining cells would be unlikely to produce a depth distribution that resembles reality. This approach, if spatial correlation among cells is properly incorporated in the analysis, may be useful to assess the effect of the soil depth variability, at a particular location, to the infiltration at that location in the model domain.

Alternatively, if more general information, such as net infiltration over the entire model domain, is sufficient, then an appropriate bulk parameter value may be determined that is applied over the entire set of model cells belonging to a particular soil depth class. Appropriate bulk parameter means that when all cells in a class are assigned the same value, the net infiltration averaged across the entire depth class is the same as if the soil depths in the class were allowed to vary spatially (as occurs in nature) and were then averaged to determine the mean or median infiltration.

The appropriate bulk soil depth value approach would use a further simplification of the model in which a single representative value (that may be uncertain) is used to characterize soil depth for all cells in a soil depth class. This approach would simplify calculations and would be satisfactory if infiltration values from the low end of the depth range tend to be cancelled out by values at the high end of the range, thus producing a trend toward the value predicted by a representative value when a large number of cells are considered. Implementation of this approach also requires a careful assessment because infiltration rates may change dramatically with depth within the depth range, so that cells from one segment of the distribution dominate infiltration and, thus, result in a different total infiltration value compared with infiltration from another segment of the distribution. This possible nonlinear or nonsymmetrical infiltration response implies that a simple arithmetic mean value may not be the appropriate bulk parameter value to assign to all cells in that cell class.

The following paragraphs discuss some considerations when choosing values for soil depth, if the approach used is that of a single, representative value.

\section{$\underline{\text { Soil Depth Class } 1}$}

This depth class represents very thick alluvial soils, described by a uniform distribution with lower and upper bound values of 40 and $150 \mathrm{~m}$, respectively. Because this class represents 
depths above the threshold where infiltration into the bedrock is expected, using a representative value equal to the mean for the class of $95 \mathrm{~m}$ is appropriate. Because soil depths in this class are large and infiltration is expected to be small, the specific value chosen within this range is unlikely to cause a significant change to predicted infiltration.

\section{$\underline{\text { Soil Depth Class } 2}$}

This depth class represents moderately alluvial deep soils that range in depth from $0.5 \mathrm{~m}$ to about $50 \mathrm{~m}$. This class is intended to include the value where soil depth is sufficient to prohibit infiltration of water to the soil-bedrock contact, except in some channels, because the soils have sufficient storage capacity to retain precipitation in the root zone where it is subject to evapotranspiration. Therefore, the treatment of soil depth in this class is dependent on the value assumed or calculated by the model for this infiltration threshold related to the climate state of interest. It is expected that infiltration in the Soil Depth Class 2 areas is most likely to occur where soil thickness is small. Consequently, the appropriate bulk parameter value will lie closer to the small soil thickness portion of the distribution (Table 6-10), rather than near the large soil thickness values. A separate analysis may need to be conducted to determine the appropriate bulk soil thickness for Soil Depth Class 2.

\section{$\underline{\text { Soil Depth Class } 3}$}

This depth class represents areas of thicker footslope colluvium that occur intermittently in the area. The data are represented by a lognormal distribution with an estimated population mean soil depth of $3.25 \mathrm{~m}$ and a sample median of $2.07 \mathrm{~m}$, which is also the estimated population median; only one value is larger than $5.18 \mathrm{~m}$ (Figure 6-15 and Table 6-7). The depth in Soil Depth Class 3 will be small where it contacts Soil Depth Class 4, but increases where it contacts other soil depth classes, primarily Soil Depth Class 2. The majority of infiltration through Soil Depth Class 3 will occur where the depth is small. The appropriate effective uniform depth for Soil Depth Class 3 is a value that allows for the same total infiltration, through all of Soil Class 3, as occurs through the spatially variable material that exists in nature.

\section{$\underline{\text { Soil Depth Class } 4}$}

This shallow depth class represents colluvial-covered upland slopes and plateaus. The data are represented by a lognormal distribution with an estimated population mean of $0.4 \mathrm{~m}$ and a sample median of $0.25 \mathrm{~m}$. Because this depth class is thin, infiltration may occur throughout the entire area of Soil Depth Class 4. Uncertainty in the effective uniform soil depth for this class is presented in Table 6-10 for both the population mean and the median. Users of the data will need to assess the appropriate effective uniform value and evaluate the effect of any uncertainty in that value.

\section{$\underline{\text { Soil Depth Class } 5}$}

This class represents exposed bedrock in the area that does not have soil cover. Therefore, all cells in this class should be assigned a zero soil depth value. 


\section{CONCLUSIONS}

\subsection{TECHNICAL ADEQUACY OF THE EXISTING SOIL DEPTH FILE}

Because of the limited availability of direct surficial deposit thickness measurements, any method of estimating thickness over the model area is subject to uncertainty. Using soil classification as a surrogate for depth information is considered the best approach given the available information. This evaluation, therefore, concludes that the general approach used in the development of SOILMAP6.INP (USGS 2000 [DIRS 175858], folder Soilmap6) is acceptable for previous versions of the infiltration model (BSC 2004 [DIRS 170007]); USGS 2000 [DIRS 123650]; USGS 2001 [DIRS 154674]; USGS 2001 [DIRS 160355]; USGS 2003 [DIRS 166518]). The approach, however, results in values that are subject to uncertainty at a detailed level, such as depth estimate values for specific model cells calculated from equations based on topographic slopes.

While SOILMAP6.INP (USGS 2000 [DIRS 175858], folder Soilmap6) provides a reasonable, generalized model of soil thickness in the vicinity of Yucca Mountain as defined by general soil depth codes (Figures 6-3 and 6-8), the detailed calculations of the depth for each grid cell based on topographic slope are not supported by available data. The natural variability in soil depth at all scales is too great to allow correlations between slope and soil to be significant. The effect from these detailed slope-based soil depth calculations on the output of previous versions of the infiltration model (BSC 2004 [DIRS 170007]); USGS 2000 [DIRS 123650]; USGS 2001 [DIRS 154674]; USGS 2001 [DIRS 160355]; USGS 2003 [DIRS 166518]) is beyond the scope of this analysis.

Some elements of the production of SOILMAP6.INP (USGS 2000 [DIRS 175858], folder Soilmap6) are not fully transparent. The methods used to define the original soil depth codes, and the development of equations used to calculate depth, are considered to be based on author judgment, which is not well documented. There is also a difference between the boundaries of the soil depth codes defined by the depth equations and those defined in the infiltration model report (BSC 2004 [DIRS 170007]). These issues make it difficult to completely assess the process used to develop SOILMAP6.INP (USGS 2000 [DIRS 175858], folder Soilmap6).

Although the infiltration model report (BSC 2004 [DIRS 170007]) identifies and documents the creation of SOILMAP6.INP (USGS 2000 [DIRS 175858], folder Soilmap6), it does not provide the location of the file. DTN: MO0512SPASURFD.000 [DIRS 175870], containing SOILMAP6.INP (USGS 2000 [DIRS 175858], folder Soilmap6), was created as output from the infiltration model report (BSC 2004 [DIRS 170007]) to provide a traceable link to the actual input file. These issues are documented in Condition Report Number 6334.

While SOILMAP6.INP (USGS 2000 [DIRS 175858], folder Soilmap6) is considered acceptable for current and previous versions of an infiltration model (BSC 2004 [DIRS 170007]); USGS 2000 [DIRS 123650]; USGS 2001 [DIRS 154674]; USGS 2001 [DIRS 160355]; USGS 2003 [DIRS 166518]), use of the file in future versions is not recommended because of the traceability and transparency issues. 


\subsection{CONCLUSIONS BASED ON THE SUMMARY OF EXISTING DATA}

Future infiltration modeling should use a soil depth file that is traceable to data sources available for the model area. This analysis supplies an alternate evaluation of soil depth that can be used as such a source. The evaluation herein relies on a simplified approach that uses qualified data from boreholes, field surficial deposits mapping, and the GFM (BSC 2004 [DIRS 170029], Figure 6-10). The evaluation divides the infiltration model area into five soil depth classes (Figure 6-12) and offers a set of distributions representing available data (Table 6-10) that can be used to estimate soil depths for model grid cells.

The soil depth class for each grid cell in the model area and a listing of the soil depth distributions are included in output DTN: MO0606SPASDFIM.005.

The soil depth data used herein are sufficient to provide input to an infiltration model when data limitations and uncertainties are recognized. Users of output DTN: MO0606SPASDFIM.005 for future infiltration modeling should consider the requirements and sensitivities of their model in determining its utility.

The grid file and distributions in output DTN: MO0606SPASDFIM.005 can be used to randomly sample values from the soil depth classes shown in the file and assign values to particular grid cells.

Output DTN: MO0606SPASDFIM.005 does not attempt to coordinate values used with adjacent cells that were based on slope or other factors. Such an approach can result in a pattern in which adjacent cells may have significantly different soil depth values because of the uncertainty resulting from a limited set of field measurements and because of the known high natural variability of soil depths in shallower depth classes. The output is considered a reasonable representation of soil depth variability across the model area based on currently available data.

Estimates made by SOILMAP6.INP (USGS 2000 [DIRS 175858], folder Soilmap6) and output DTN: MO0606SPASDFIM.005 are compared for the shallow soil class to illustrate the differences between the two approaches. Figure 7-1 shows the distribution of 42,851 grid cell values for Soil Depth Code 1 (Figure 6-3) taken from the portion of the data analyzed (Section 6.1.2) to test the depth calculations. These values are compared to a hypothetical distribution based on the sample mean and the sample standard deviation for Soil Depth Class 4 (Table 6-10), but are scaled up to the same amount of values from SOILMAP.INP (USGS 2000 [DIRS 175858], folder Soilmap6) to provide the comparison.

The mean for the SOILMAP6.INP sample $(0.26 \mathrm{~m})$ (USGS 2000 [DIRS 175858], folder Soilmap6) is similar to the sample median $(0.25 \mathrm{~m})$ and the anti-log of the sample mean of the log-depth values $(0.27 \mathrm{~m})$ for Soil Depth Class 4 ; the values, however, are distributed differently. 


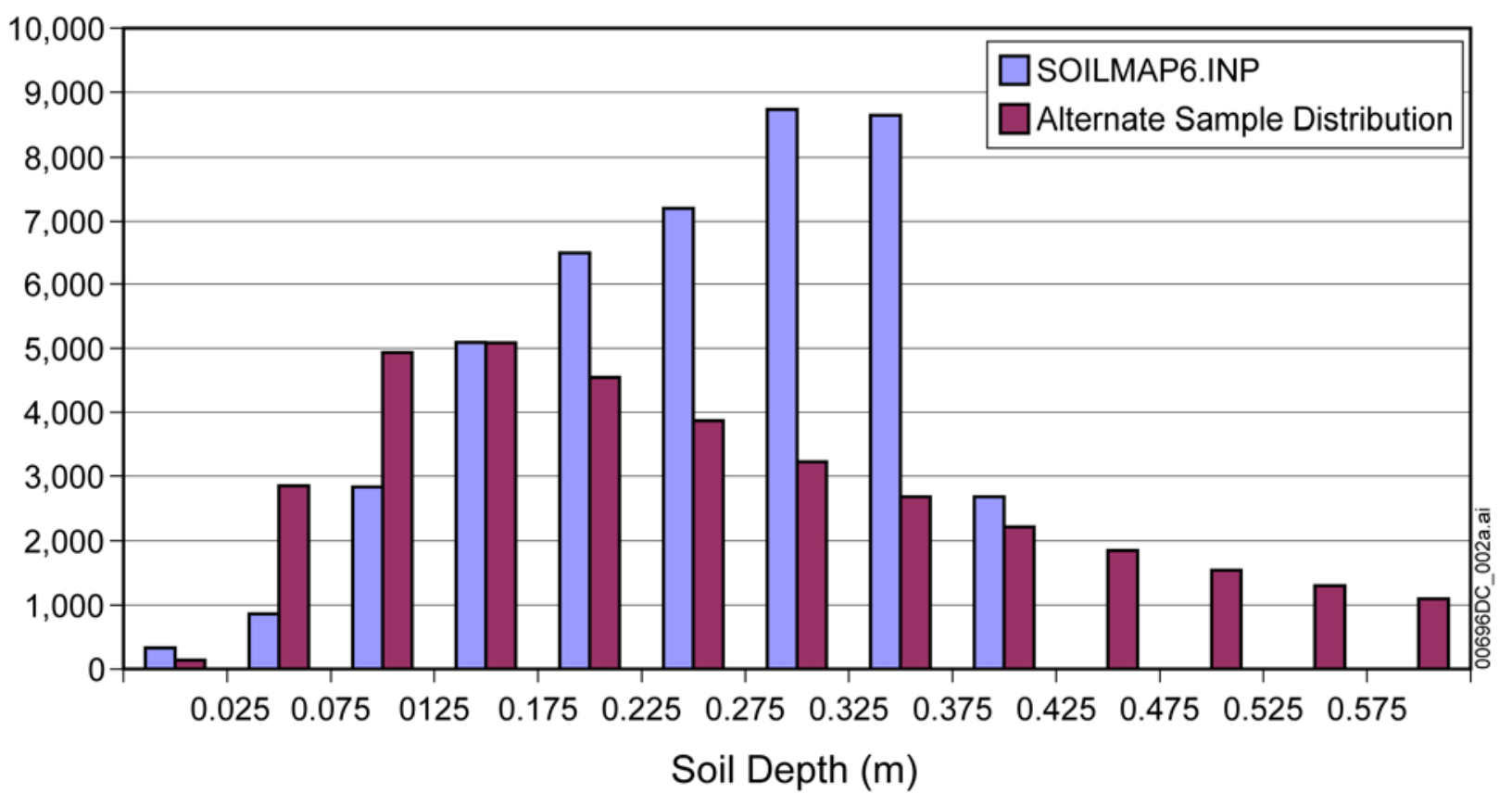

Sources: Table 6-10; Appendix B, Sections B.1.2 and B.1.13 and Section 7 comparison.xls, worksheet 'Figure 7-1'.

NOTE: The hypothetical distribution, based on the sample mean and on the sample standard deviation for Soil Depth Class 4 (Table 6-10), uses the same total number of values as those used in the SOILMAP.INP sample (USGS 2000 [DIRS 175858], folder Soilmap6).

Figure 7-1. Comparison of Values Calculated for Soil Depth Code 1 in SOILMAP6.INP and Soil Depth Class 4

\subsection{SUMMARY OF THIS ANALYSIS}

This analysis addresses work scope elements (Section 1) and presents the following findings:

- The technical adequacy of the soil depth file used in the infiltration model report (BSC 2004 [DIRS 170007]) was evaluated through a review of supporting documentation and corroborating data.

- The findings from the evaluation of the existing file are:

- The method used to calculate soil depths for each grid cell in the model is not well-documented or traceable

- The general soil depth codes and depth ranges used are reasonable

- The specific depth calculations for each grid cell based on topographic slope are not corroborated by the existing data.

- After an evaluation of SOILMAP6.INP (USGS 2000 [DIRS 175858], folder Soilmap6), it was determined that an alternate soil depth file should be created. This is consistent with the resolution plan to reperform the preparation of the soil depth file for issues noted in the evaluation, using available data to provide an alternate source for future modeling. 
- This analysis generated an alternate soil depth file that assigns a soil depth class to each grid cell in the model area (Figure 6-12) and provides distributions characterizing soil depth for each of the classes (Table 6-10). These files are located in output DTN: MO0606SPASDFIM.005.

- There is significant uncertainty involved in soil depth characterizations because of significant natural variability at all scales in soil depth and because of the limited size of data sets that provide soil depth information. Users of the results of this analysis need to carefully consider the uncertainties of the results.

- This analysis addresses the criteria identified in Section 4.2 as listed in Table 7-1.

Table 7-1. Mapping of Yucca Mountain Review Plan Acceptance Criteria and Soil Depth Summary

\begin{tabular}{|c|c|c|}
\hline Acceptance Criteria & Subcriteria & How Addressed \\
\hline \multirow[t]{3}{*}{$\begin{array}{l}\text { Acceptance Criterion 2: } \\
\text { Data are sufficient for } \\
\text { model justification } \\
\text { (NRC 2003 } \\
\text { [DIRS 163274], } \\
\text { Section 2.2.1.3.5.3) }\end{array}$} & $\begin{array}{l}\text { Climatological and hydrological values used in } \\
\text { the license application (e.g., time of onset of } \\
\text { climate change, mean annual temperature, mean } \\
\text { annual precipitation, mean annual net infiltration, } \\
\text { etc.) are adequately justified. Adequate } \\
\text { descriptions of how the data were used, } \\
\text { interpreted, and appropriately synthesized into } \\
\text { the parameters are provided. }\end{array}$ & Not applicable for soil depth \\
\hline & $\begin{array}{l}\text { The effects of fracture properties, fracture } \\
\text { distributions, matrix properties, heterogeneities, } \\
\text { time-varying boundary conditions, } \\
\text { evapotranspiration, depth of soil cover, and } \\
\text { surface-water runoff and run-on are considered, } \\
\text { such that net infiltration is not underestimated. }\end{array}$ & $\begin{array}{l}\text { The variation in the soil cover depth } \\
\text { establishes a reasonable and realistic } \\
\text { estimate, based on available data, for } \\
\text { use in modeling. The limited data set } \\
\text { used herein does introduce some } \\
\text { uncertainty into the estimates, which } \\
\text { are discussed and incorporated into } \\
\text { the analyses as discussed in } \\
\text { Sections 6.2.1 and 6.2.2. }\end{array}$ \\
\hline & $\begin{array}{l}\text { Sensitivity or uncertainty analyses are performed } \\
\text { to assess data sufficiency and determine the } \\
\text { possible need for additional data. }\end{array}$ & $\begin{array}{l}\text { Uncertainty in the soil depth values } \\
\text { discussed and incorporated in } \\
\text { recommendations as discussed in } \\
\text { Section 6.2.2. }\end{array}$ \\
\hline \multirow{3}{*}{$\begin{array}{l}\text { Acceptance Criterion } 3: \\
\text { Data uncertainty is } \\
\text { characterized and } \\
\text { propagated through } \\
\text { the model abstraction } \\
\text { (NRC 2003 } \\
\text { [DIRS 163274], } \\
\text { Section 2.2.1.3.5.3) }\end{array}$} & $\begin{array}{l}\text { Models use parameter values, assumed ranges, } \\
\text { probability distributions, and bounding } \\
\text { assumptions that are technically defensible, } \\
\text { reasonably account for uncertainties and } \\
\text { variabilities, and do not result in an } \\
\text { under-representation of the risk estimate. }\end{array}$ & $\begin{array}{l}\text { Uncertainties and variability included } \\
\text { in derivation of recommended values } \\
\text { to provide a reasonable parameter } \\
\text { estimate are discussed in } \\
\text { Sections } 6.2 .1 \text { and } 6.2 .2\end{array}$ \\
\hline & $\begin{array}{l}\text { The technical bases for the parameter values } \\
\text { used in this abstraction are provided. }\end{array}$ & $\begin{array}{l}\text { Available site-specific data identified } \\
\text { and evaluated in the analysis as } \\
\text { discussed in Section } 4 .\end{array}$ \\
\hline & $\begin{array}{l}\text { Possible statistical correlations are established } \\
\text { between parameters in this abstraction. An } \\
\text { adequate technical basis or bounding argument } \\
\text { is provided for neglected correlations. }\end{array}$ & $\begin{array}{l}\text { Statistical correlations established } \\
\text { and evaluated in generating } \\
\text { recommended values. Basis for } \\
\text { neglected correlation between } \\
\text { slope and depth provided. These } \\
\text { correlations and the basis are } \\
\text { discussed in Sections } 6.2 .1 \\
\text { and 6.2.2. }\end{array}$ \\
\hline
\end{tabular}




\section{INPUTS AND REFERENCES}

\subsection{DOCUMENTS CITED}

170029 BSC (Bechtel SAIC Company) 2004. Geologic Framework Model (GFM2000). MDL-NBS-GS-000002 REV 02. Las Vegas, Nevada: Bechtel SAIC Company. ACC: DOC.20040827.0008.

170007 BSC 2004. Simulation of Net Infiltration for Present-Day and Potential Future Climates. MDL-NBS-HS-000023 REV 00. Las Vegas, Nevada: Bechtel SAIC Company. ACC: DOC.20041109.0004.

175539 BSC 2005. Q-List. 000-30R-MGR0-00500-000-003. Las Vegas, Nevada: Bechtel SAIC Company. ACC: ENG.20050929.0008.

176107 BSC 2006. Technical Work Plan for: Infiltration Model Assessment, Revision, and Analyses of Downstream Impacts. TWP-NBS-HS-000012 REV 01. Las Vegas, Nevada: Bechtel SAIC Company. ACC: DOC.20060110.0002.

176335 BSC 2006. Data Analysis for Infiltration Modeling: Development of Soil Units and Associated Hydraulic Parameter Values. ANL-NBS-HS-000055 REV 00. Las Vegas, Nevada: Bechtel SAIC Company.

150920 CRWMS M\&O 2000. Software Baseline Request for LV-1999-006, ARCINFO V7.2.1. 10033-7.2.1-00. Las Vegas, Nevada: CRWMS M\&O. ACC: MOL.20000519.0018.

175970 Dixon, W.R. 1992. "Proof of Completion Information for Well J-12." Letter from W.R. Dixon (DOE/YMSCO) to G.M. Bilyeu (Thiel, Winchell \& Associates, Inc.), November 17, 1992, POCD:DLS-993, with enclosures.

ACC: NNA.19921203.0048; MOL.19980218.0561.

176873 EPA (U.S. Environmental Protection Agency) 2002. Calculating Upper Confidence Limits for Exposure Point Concentrations at Hazardous Waste Sites. OSWER 9285.6-10. Washington, D.C.: U.S. Environmental Protection Agency, Office of Emergency and Remedial Response. ACC: MOL.20060419.0263.

175968 Gertz, C.P. 1992. "Preliminary Field Composite Borehole Log." Correspondence from C.P. Gertz (DOE/YMSCO) to C.H. Johnson (State of Nevada), P. Niedzielski-Eichner (Nye County), D.A. Bechtel (Clark County), A.C. Douglas (City of Las Vegas), and P.T. Presholt (NRC), March 11, 1992, RSED:USC-2227, with enclosure. ACC: NNA.19920320.0004.

163705 Gilbert, R.O. 1987. Statistical Methods for Environmental Pollution Monitoring. New York, New York: John Wiley \& Sons. TIC: 252619. 
163274 NRC (U.S. Nuclear Regulatory Commission) 2003. Yucca Mountain Review Plan, Final Report. NUREG-1804, Rev. 2. Washington, D.C.: U.S. Nuclear Regulatory Commission, Office of Nuclear Material Safety and Safeguards. TIC: 254568.

176569 Sanchez, A. 2006. Conducting Confirmatory Field Observations for Special Infiltration Project, partial submittal. Scientific Notebook SN-M\&O-SCI-053-V1. Pages 1-83 ACC: MOL.20060306.0186.

165862 Stonestrom, D.A.; Prudic, D.E.; Laczniak, R.J.; Akstin, K.C.; Boyd, R.A.; and Henkelman, K.K. 2003. Estimates of Deep Percolation Beneath Native Vegetation, Irrigated Fields, and the Amargosa-River Channel, Amargosa Desert, Nye County, Nevada. Open-File Report 03-104. Denver, Colorado: U.S. Geological Survey. TIC: 255088.

176601 Taylor, E.M. 2001. Empirical Calculation of Soil Thickness Based on Field Measurements, final submittal. Scientific Notebook SN-USGS-SCI-113-V1. Pages a-61 ACC: MOL.20020320.0368.

175859 USDA (United States Department of Agriculture) 1994. Keys to Soil Taxonomy. 6th Edition. Washington, D.C.: United States Department of Agriculture, Soil Conservation Service. ACC: MOL.20060123.0278.

175858 USGS (U.S. Geological Survey) 2000. Copy of GEOINPUT -1 (BLOCKR7, GEOMAP7, GEOMOD4, AND SOILMAP6) Attachment to Analysis Report ANL-NBS-HS-000032, Revision 0 (Includes 2 CDs and a Special Instruction Sheet). ANL-NBS-HS-000032 REV 00. Denver, Colorado: U.S. Geological Survey. ACC: MOL.20050801.0163.

123650 USGS 2000. Simulation of Net Infiltration for Modern and Potential Future Climates. ANL-NBS-HS-000032 REV 00. Denver, Colorado: U.S. Geological Survey. ACC: MOL.20000801.0004.

154674 USGS 2001. Simulation of Net Infiltration for Modern and Potential Future Climates. ANL-NBS-HS-000032 REV 00 ICN 01. Denver, Colorado: U.S. Geological Survey. ACC: MOL.20010405.0002.

160355 USGS 2001. Simulation of Net Infiltration for Modern and Potential Future Climates. ANL-NBS-HS-000032 REV 00 ICN 02. Denver, Colorado:

U.S. Geological Survey. ACC: MOL.20011119.0334.

166518 USGS 2003. Simulation of Net Infiltration for Modern and Potential Future Climates. ANL-NBS-HS-000032 REV 00 ICN 02, Errata 002. Denver, Colorado: U.S. Geological Survey. ACC: MOL.20011119.0334; DOC.20031014.0004; DOC.20031015.0001. 
175967 YMP (Yucca Mountain Site Characterization Project) 1992. Yucca Mountain Site Characterization Project Preliminary Field Composite Borehole Log. Bore Hole ID: USW UZ-N15. Las Vegas, Nevada: Yucca Mountain Site Characterization Office. ACC: MOL.19980304.0465.

176008 YMP 1992. Yucca Mountain Site Characterization Project Preliminary Field Composite Borehole Log. Bore Hole ID: USW UZ-N16. Las Vegas, NV: Yucca Mountain Site Characterization Office. ACC: MOL.19980304.0467.

176010 YMP 1992. Yucca Mountain Site Characterization Project Preliminary Field Composite Borehole Log - Borehole ID: USW UZ-N17. Las Vegas, Nevada: Yucca Mountain Site Characterization Office. ACC: MOL.19980304.0469.

175965 YMP 1992. Yucca Mountain Site Characterization Project Preliminary Field Composite Borehole Log. Bore Hole ID: USW UZN-36. Las Vegas, Nevada: Yucca Mountain Site Characterization Office. ACC: MOL.19980316.0393.

175923 YMP 1992. Yucca Mountain Site Characterization Project Sample Management Facility Lithologic Log. Borehole ID: UE-25 UZ-16. Las Vegas, Nevada: Yucca Mountain Site Characterization Office. ACC: MOL.19950330.0388.

176592 YMP 1993. Construction of Access Road for Borehole UE-25 NRG-4. Job Package 93-02. Las Vegas, Nevada: Yucca Mountain Site Characterization Office. ACC: DRC.19960709.0002.

175962 YMP 1993. Main Drift Tunnel Alignment As/Built NRG-7. YMP 52/22. Las Vegas, Nevada: Yucca Mountain Site Characterization Office. ACC: NNA.19940218.0070.

175963 YMP 1993. Yucca Mountain Site Characterization Project Lithologic Log Borehole ID: USW NRG-7. Las Vegas, Nevada: Yucca Mountain Site Characterization Office. ACC: MOL.19950119.0222.

176591 YMP 1994. Construction of Drill Pad and Refurbishment of Access Road for Borehole USW UZ-7a. Job Package 94-14. Las Vegas, Nevada: Yucca Mountain Site Characterization Office. ACC: DRC.19960729.0031.

176589 YMP 1994. Field Activities Management Report Format, Construction of Access Road \& Drill Pad for Borehole USW5D7, Construction of Access Road \& Drill Pad, July 8, 1994. Las Vegas, Nevada: Yucca Mountain Site Characterization Office. ACC: DRC.19960729.0007.

176590 YMP1994. Survey Support USW SD-7 Topo Drill Pad. Las Vegas, Nevada: Yucca Mountain Site Characterization Office. ACC: DRC.19960729.0029. 
175930 YMP 1994. Yucca Mountain Site Characterization Project Composite Borehole Log. Borehole ID: UE-25NRG\#2C. Las Vegas, Nevada: Yucca Mountain Site Characterization Office. ACC: MOL.19940719.0031.

172113 YMP 1995. Composite Borehole Log, Borehole ID: USW UZ-14. Las Vegas, Nevada: Yucca Mountain Site Characterization Office.

ACC: DRC.19960702.0263.

\subsection{CODES, STANDARDS, REGULATIONS, AND PROCEDURES}

176544 10 CFR 63. 2006 Energy: Disposal of High-Level Radioactive Wastes in a Geologic Repository at Yucca Mountain, Nevada. Internet Accessible.

IT-PRO-0011, Software Management.

LP-3.15Q-BSC, Managing Technical Product Inputs.

LP-SIII.9Q-BSC, Scientific Analyses.

LS-PRO-0203, Q-List and Classification of Structures, Systems, and Components.

\subsection{SOURCE DATA, LISTED BY DATA TRACKING NUMBER}

158000 GS000308314211.002. Stratigraphic Contacts for Boreholes UE-25 A\#1, UE-25 A\#4, USW G-1, USW G-2, USW H-5, USW SD-9, USW SD-12 and USW VH-2. Submittal date: 04/28/2000.

176317 GS011208312212.004. SN-USGS-SCI-113 V1: Empirical Calculation of Soil Thickness Based on Field Measurements. Submittal date: 01/24/2002.

164561 GS030783114233.001. Geotechnical Borehole Logs for the Waste Handling Building, Yucca Mountain Project, Nevada Test Site, Nevada, Version 7/16/03. Submittal date: 07/23/2003.

175972 GS910808312212.001. Geohydrologic Data Collected from Shallow Neutron-Access Boreholes and Resultant Preliminary Geohydrologic Evaluations, Yucca Mountain Area, Nye County, Nevada. Submittal date: 08/09/1991.

150007 GS931008314211.037. Graphical Lithologic Log of Borehole NRG-3 (UE-25 NRG\#3), Version 1.0. Submittal date: 10/07/1993.

150009 GS931008314211.039. Graphical Lithologic Log of Borehole NRG-2 (UE-25 NRG\#2), Yucca Mountain, Nevada. Submittal date: 10/07/1993.

150010 GS931008314211.045. Graphical Lithologic Log of Bore Hole USW NRG-6. Submittal date: 10/07/1993. 
150011 GS931108314211.041. Graphical Lithologic Log of Borehole NRG-2B (UE-25 NRG\#2B), Yucca Mountain, Nevada. Submittal date: 10/07/1993.

150012 GS931208314211.046. Graphical Lithologic Log of Borehole UE-25 NRG\#1. Submittal date: $11 / 22 / 1993$.

110313 GS931208314211.047. Graphical Lithologic Log of Borehole UE-25 UZ\#16. Submittal date: 11/22/1993.

160344 GS940108315142.004. Draft Surficial Deposits Map of the Northeast Quarter of the Busted Butte 7.5-Minute Quadrangle. Submittal date: 12/22/1993.

160345 GS940108315142.005. Draft Surficial Deposits Map of the Southern Half of the Topopah Spring NW 7.5-Minute Quadrangle. Submittal date: 12/22/1993.

145577 GS940208314211.002. Table of Contacts in Boreholes USW UZ-N62. Submittal date: $02 / 01 / 1994$.

152565 GS940208314211.003. Table of Contacts in Boreholes USW UZ-N53, USW UZ-N54, and USW UZ-N55. Submittal date: 02/01/1994.

145579 GS940208314211.004. Table of Contacts in Borehole USW UZ-N27. Submittal date: $02 / 10 / 1994$.

152557 GS941008314211.051. Graphical Lithologic Log for Borehole USW UZ-N31. Submittal Date: 10/19/1994.

152563 GS940208314211.005. Table of Contacts in Boreholes USW UZ-N31, UZ-N32, UZ-N37. Submittal date: 02/10/1994.

152555 GS940208314211.006. Table of Contacts in Boreholes USW UZ-N33 and USW UZ-N34. Submittal date: 02/10/1994.

155533 GS940208314211.007. Table of Contacts in Borehole USW UZ-N35. Submittal date: $02 / 10 / 1994$.

145581 GS940208314211.008. Table of Contacts in Boreholes USW UZ-N57, UZ-N58, UZ-N59, and UZ-N61. Submittal date: 02/10/1994.

145588 GS940308314211.011. Table of Contacts for the Tiva Canyon Tuff in Borehole USW UZ-N38. Submittal date: 03/10/1994.

146474 GS940308314211.016. Table of Contacts for the Tiva Canyon Tuff in Borehole USW UZ-N64. Submittal date: 03/28/1994.

155534 GS940308314211.017. Table of Contacts for the Tiva Canyon Tuff in Borehole UE-25 UZN\#63. Submittal date: 03/28/1994. 
145589 GS940308314211.018. Table of Contacts for the Tiva Canyon Tuff in Borehole USW UZ-N36. Submittal date: 03/28/1994.

160346 GS940708315142.008. Draft Surficial Deposits Map of the Northwest Quarter of the Busted Butte 7.5-Minute Quadrangle, Nye County, Nevada. Submittal date: $07 / 27 / 1994$.

160347 GS950408315142.004. Draft Map of the Surficial Deposits of the Southern Half of the Busted Butte 7.5' Quadrangle, Nye County, Nevada. Submittal date: 04/03/1995.

146299 GS960408312212.005. Preliminary Surficial Materials Properties Map: Soils of the Yucca Mountain Area, NV. Submittal date: 04/18/1996.

124829 GS960508312212.007. Estimated Distribution of Geomorphic Surfaces and Depth to Bedrock for the Southern Half of the Topopah Spring NW 7.5 Minute Quadrangle and the Entire Busted Butte 7.5 Minute Quadrangle. Submittal date: 04/21/1996.

176939 GS980183117462.001. Trench Logs and Unit Descriptions for Solitario Canyon Fault Trenches T8 (TR8), T8A (TR8A), SCF-T1, SCF-T1A, SCF-T2, SCF-T3, and SCF-T4. Submittal date: 01/16/1998.

152562 MO0002GSC00068.000. UE-25 NRG-1 Drill Hole Resurvey for Comparison. Submittal date: $02 / 10 / 2000$.

152554 MO0004QGFMPICK.000. Lithostratigraphic Contacts from MO9811MWDGFM03.000 to be Qualified Under the Data Qualification Plan, TDP-NBS-GS-000001. Submittal date: 04/04/2000.

153777 MO0012MWDGFM02.002. Geologic Framework Model (GFM2000). Submittal date: $12 / 18 / 2000$.

157481 MO0101SEPBGLOG.000. Sample Management \& Drilling Department Records Package for Borehole Records (Geologic Logs) from the "Geotechnical Field Investigation for the Waste Handling Building," FWP-SB-00-003. Submittal date: $01 / 10 / 2001$.

157304 MO0103GSC01031.000. As-Built Borehole Locations Waste Handling Building WHB Site Investigation. Submittal date: 03/07/2001.

155539 MO0106STRATHFM.004. Lithologic Description of Drill Hole USW GU-3 and USW G-3. Submittal date: 06/27/2001.

155589 MO0106STRATHFM.028. Generalized Lithologic Log for Test Well USW H-1. Submittal date: 06/27/2001. 
155590 MO0106STRATHFM.029. Lithologic Log for Drill-Hole UE-25 P\#1. Submittal date: $06 / 27 / 2001$.

155591 MO0106STRATHFM.030. Lithologic Log of Drill-Hole USW G-1. Submittal date: $06 / 27 / 2001$.

155592 MO0106STRATHFM.031. Lithologic Description of Exploratory Drill Hole USW G-2. Submittal date: 06/27/2001.

168375 MO0203GSC02034.000. As-Built Survey of Nye County Early Warning Drilling Program (EWDP) Phase III Boreholes NC-EWDP-10S, NC-EWDP-18P, and NC-EWDP-22S - Partial Phase III List. Submittal date: 03/21/2002.

175917 MO0210SMFGLOG3.000. Sample Management Facility Geologic Logs for Nye County Early Warning Drilling Program (EWDP) Phase III. Submittal date: $10 / 08 / 2002$.

175919 MO0506GSC05047.000. As-Built Location for Surface Boreholes. Submittal date: $06 / 20 / 2005$.

175870 MO0512SPASURFD.000. Input File on Surficial Deposits Depth for Infiltration Modeling. Submittal date: 12/05/2005.

109060 MO9811COV98591.000. Coverage: TOPO20S. Submittal date: 11/09/1998.

103793 MO9902ASBUBOLC.000. As-Built Location of UE-25 RF\#13 Borehole. Submittal date: 02/23/1999.

103790 MO9903BLOGSOIL.000. UE-25 RF\#13 Borehole Log of Soil and Other Materials Above Bedrock. Submittal date: 03/05/1999.

176571 MO9903COV96274.000. Coverage: Soils. Submittal date: 03/29/1999.

165921 MO9905LUSWWT24.000. Surveyed Location USW WT-24. Submittal date: $05 / 07 / 1999$.

109059 MO9906GPS98410.000. Yucca Mountain Project (YMP) Borehole Locations. Submittal date: 06/23/1999.

165922 MO9912GSC99492.000. Surveyed USW SD-6 As-Built Location. Submittal date: 12/21/1999.

175927 SNF29041993002.034. Yucca Mountain Site Characterization Project Geology and Rock Structure Log for Drillhole UE25 NRG-1, Rev. 1. Submittal date: $02 / 06 / 1995$. 
175928 SNF29041993002.036. Yucca Mountain Site Characterization Project Geology and Rock Structure Log For Drillhole UE25 NRG-2A, Rev. 1. Submittal date: 02/06/1995.

175958 SNF29041993002.038. Yucca Mountain Site Characterization Project Geology and Rock Structure for Drillhole UE25 NRG-3, Rev. 1. Submittal date: 02/06/1995.

175959 SNF29041993002.039. Yucca Mountain Site Characterization Project Geology and Rock Structure Log For Drillhole UE25 NRG-4, Rev. 1. Submittal date: 02/06/1995.

175960 SNF29041993002.040. Yucca Mountain Site Characterization Project Geology and Rock Structure Log For Drillhole UE25 NRG-5, Rev. 1. Submittal date: 02/06/1995.

166437 SNF29041993002.042. Yucca Mountain Site Characterization Project Geology and Rock Structure Log for Drillhole USW NRG-7/7A, Rev. 1. Submittal date: 02/06/1995.

107239 SNF40060198001.001. Unsaturated Zone Lithostratigraphic Contacts in Borehole USW WT-24. Submittal date: 10/15/1998.

107372 SNF40060298001.001. Unsaturated Zone Lithostratigraphic Contacts in Borehole USW SD-6. Submittal date: 10/15/1998.

105187 SNT02052794001.003. Geologic Core Logs for USW SD-9, Version 2.0. Submittal date: $01 / 29 / 1996$.

175971 TM0000000000CL.005. UE-25 JF-3 Composite Borehole Log. Submittal date: $10 / 13 / 1995$.

175918 TM0000000SD7RS.006. USW SD-7 Video Tapes and Composite Borehole Log To a Total Depth Of 2675.1'. Submittal date: 02/13/1996.

175924 TM000000SD12RS.014. USW SD-7 Composite Borehole Log and Sample Weight Logs. Submittal date: 02/27/1996.

160826 TM000000UZ7ARS.001. USW UZ-7A Shift Drilling Summaries, Lithologic Logs, Structural Logs, Weight Logs, and Composite Borehole Log from 0.0' to 770.0'. Submittal date: 09/05/1995. 
Data Analysis for Infiltration Modeling: Technical Evaluation of Previous Soil Depth Estimation Methods and Development of Alternate Parameter Values

\subsection{OUTPUT DATA, LISTED BY DATA TRACKING NUMBER}

MO0606SPASDFIM.005. Soil Depth Input File for Use in Infiltration Modeling. Submittal date: $06 / 08 / 2006$.

\subsection{SOFTWARE CODES}

157019 2000. Software Code: ARCINFO. V.7.2.1. SGI, IRIX 6.5. STN: 10033-7.2.1-00.

139422 USGS (U.S. Geological Survey) 2001. Software Code: INFIL. V2.0. PC, Windows NT 4.0. 10307-2.0-00. 
Data Analysis for Infiltration Modeling: Technical Evaluation of Previous Soil Depth Estimation Methods and Development of Alternate Parameter Values

INTENTIONALLY LEFT BLANK 
APPENDIX A

BOREHOLES AND SURFICIAL DEPOSIT DEPTH MEASUREMENTS 


\section{BOREHOLES AND SURFICIAL DEPOSIT DEPTH MEASUREMENTS}

The data listed as direct input for Borehole Soil Depth and Borehole Location (Table 4-1) are compiled into a single table (Table A-1). Table A-1 includes both qualified data used in the analysis and nonqualified data used for corroboration. Columns A through D of Table A-1 include the borehole name, Nevada State Plane coordinate northing and easting (Nevada Central zone, U.S. feet, NAD 27), and the source of the location data. Columns E through H contain the Alluvial thickness (in feet and in meters), the source of the thickness data, and a remarks column that indicates the quality status of the data. The quality status refers to both the location and Alluvial Thickness DTNs unless otherwise indicated. The qualification / verification status does not apply to other RIS records referenced. The sections of the data with qualified and unqualified data are clearly marked.

The reader should note that in some cases, such as the entry for USW UZ-N36, on page A-4, the status of qualified and verified refers to the DTN file listed; not to the record. In these cases, the DTN file does not contain the data, but rather points to the record that does contain the data. 
Table A-1. Boreholes and Surficial Deposit Depth Measurements

\begin{tabular}{|c|c|c|c|c|c|c|c|}
\hline $\begin{array}{c}\text { Borehole } \\
\text { Name }\end{array}$ & \begin{tabular}{|c|} 
State \\
Plane \\
Coordinate \\
Northing \\
\end{tabular} & \begin{tabular}{|c|} 
State \\
Plane \\
Coordinate \\
Easting
\end{tabular} & $\begin{array}{l}\text { Location Source by Author and Date, } \\
\text { or DTN, or Both }\end{array}$ & \begin{tabular}{|c|} 
Alluvial \\
$\begin{array}{c}\text { Thickness } \\
\text { (ft) }\end{array}$ \\
\end{tabular} & \begin{tabular}{|c}
$\begin{array}{c}\text { Alluvial } \\
\text { Thickness } \\
(\mathbf{m})\end{array}$ \\
\end{tabular} & $\begin{array}{c}\text { Thickness Source by } \\
\text { Author and Date, or DTN, } \\
\text { or Both }\end{array}$ & \multirow[b]{3}{*}{$\begin{array}{l}\text { DTN: MO0004QGFMPICK.000 } \\
\text { is Qualified-PVAR; } \\
\text { DTN: TM0000000SD7RS.006 } \\
\text { is qualified but not verified and } \\
\text { shows that the unconsolidated } \\
\text { material is pad fill. This data is } \\
\text { not direct input. } \\
50.2 \mathrm{ft} \text { of Pad Fill }\end{array}$} \\
\hline \multicolumn{7}{|c|}{ QUALIFIED DATA } & \\
\hline $\begin{array}{l}\text { USW } \\
\text { SD-7 }\end{array}$ & 758949.87 & 561240.26 & MO9906GPS98410.000 [DIRS 109059] ${ }^{\mathrm{a}}$ & 0.00 & 0.00 & $\begin{array}{l}\text { MO0004QGFMPICK.000 } \\
\text { [DIRS 152554]; } \\
\text { TM0000000SD7RS.006 } \\
\text { [DIRS 175918], } \\
\text { ACC: DRC. } 19960220.0044\end{array}$ & \\
\hline $\begin{array}{l}\text { USW } \\
\text { UZ-N57 }\end{array}$ & 755164.51 & 560829.83 & MO9906GPS98410.000 [DIRS 109059] $]^{\mathrm{a}}$ & 1.20 & 0.37 & $\begin{array}{l}\text { GS940208314211.008 } \\
\text { [DIRS 145581] }^{\text {[ }}\end{array}$ & Qualified and Verified \\
\hline $\begin{array}{l}\text { USW } \\
\text { UZ-N58 }\end{array}$ & 755240.39 & 560862.22 & MO9906GPS98410.000 [DIRS 109059] $]^{\mathrm{a}}$ & 22.10 & 6.74 & $\begin{array}{l}\text { GS940208314211.008 } \\
\text { [DIRS 145581] }\end{array}$ & Qualified and Verified \\
\hline $\begin{array}{l}\text { USW } \\
\text { UZ-N59 }\end{array}$ & 755321.24 & 560888.40 & MO9906GPS98410.000 [DIRS 109059] $]^{\mathrm{a}}$ & 13.10 & 3.99 & $\begin{array}{l}\text { GS940208314211.008 } \\
\text { [DIRS 145581] }\end{array}$ & Qualified and Verified \\
\hline $\begin{array}{l}\text { USW } \\
\text { UZ-N61 }\end{array}$ & 755375.92 & 560893.95 & MO9906GPS98410.000 [DIRS 109059] ${ }^{\mathrm{a}}$ & 9.80 & 2.99 & $\begin{array}{l}\text { GS940208314211.008 } \\
\text { [DIRS 145581] }^{\mathrm{e}}\end{array}$ & Qualified and Verified \\
\hline $\begin{array}{l}\text { UE-25 } \\
\text { ONC\#1 }\end{array}$ & 759257.27 & 568092.85 & MO9906GPS98410.000 [DIRS 109059] $]^{\mathrm{a}}$ & 98.00 & 29.87 & $\begin{array}{l}\text { MO0004QGFMPICK.000 } \\
\text { [DIRS 152554] }^{\mathrm{h}}\end{array}$ & Qualified and Verified \\
\hline $\begin{array}{l}\text { USW } \\
\text { UZ-N53 }\end{array}$ & 760096.24 & 564237.27 & MO9906GPS98410.000 [DIRS 109059] $]^{a}$ & 2.10 & 0.64 & $\begin{array}{l}\text { GS940208314211.003 } \\
\text { [DIRS 152565] }\end{array}$ & Qualified and Verified \\
\hline $\begin{array}{l}\text { USW } \\
\text { UZ-N54 }\end{array}$ & 760272.26 & 564262.66 & MO0506GSC05047.000 [DIRS 175919] & 19.00 & 5.79 & $\begin{array}{l}\text { GS940208314211.003 } \\
\text { [DIRS 152565] }\end{array}$ & Qualified and Verified \\
\hline $\begin{array}{l}\text { USW } \\
\text { UZ-N55 }\end{array}$ & 760503.25 & 564248.64 & MO0506GSC05047.000 [DIRS 175919] & 0.00 & 0.00 & $\begin{array}{l}\text { GS940208314211.003 } \\
\text { [DIRS 152565] }^{\text {[ }}\end{array}$ & Qualified and Verified \\
\hline $\begin{array}{l}\text { USW } \\
\text { UZ-N35 }\end{array}$ & 762264.00 & 562309.92 & MO9906GPS98410.000 [DIRS 109059] & 11.90 & 3.60 & $\begin{array}{l}\text { GS940208314211.007 } \\
\text { [DIRS 155533], } \\
\text { ACC: NNA.19940414.0078 }\end{array}$ & Qualified and Verified \\
\hline $\begin{array}{l}\text { USW } \\
\text { SD-6 }\end{array}$ & 762421.40 & 558607.70 & MO9912GSC99492.000 [DIRS 165922] & 0.00 & 0.00 & $\begin{array}{l}\text { SNF40060298001.001 } \\
\text { [DIRS 107372], } \\
\text { Table S98430_001 }\end{array}$ & Qualified and Verified \\
\hline $\begin{array}{l}\text { USW } \\
\text { UZ-N32 }\end{array}$ & 764302.62 & 562799.60 & MO9906GPS98410.000 [DIRS 109059] ${ }^{\mathrm{a}}$ & 0.00 & 0.00 & $\begin{array}{l}\text { GS940208314211.005 } \\
\text { [DIRS 152563] }^{9}\end{array}$ & Qualified and Verified \\
\hline
\end{tabular}


Table A-1. Boreholes and Surficial Deposit Depth Measurements (Continued)

\begin{tabular}{|c|c|c|c|c|c|c|c|}
\hline $\begin{array}{l}\text { Borehole } \\
\text { Name }\end{array}$ & $\begin{array}{c}\text { State } \\
\text { Plane } \\
\text { Coordinate } \\
\text { Northing }\end{array}$ & $\begin{array}{c}\text { State } \\
\text { Plane } \\
\text { Coordinate } \\
\text { Easting }\end{array}$ & $\begin{array}{c}\text { Location Source by Author and Date, } \\
\text { or DTN, or Both }\end{array}$ & $\begin{array}{l}\text { Alluvial } \\
\text { Thickness } \\
\text { (ft) }\end{array}$ & $\begin{array}{c}\text { Alluvial } \\
\text { Thickness } \\
(\mathrm{m})\end{array}$ & $\begin{array}{c}\text { Thickness Source by } \\
\text { Author and Date, or DTN, } \\
\text { or Both }\end{array}$ & Remarks \\
\hline \multicolumn{8}{|c|}{ QUALIFIED DATA (Continued) } \\
\hline $\begin{array}{l}\text { UE-25 } \\
\text { NRG\#1 }\end{array}$ & 765358.60 & 569803.06 & $\begin{array}{l}\text { MO0002GSC00068.000 [DIRS 152562], } \\
\text { /pub3/gis/baseline/test/mo0002gsc00068.zip }\end{array}$ & 6.10 & 1.86 & $\begin{array}{l}\text { SNF29041993002.034 } \\
\text { [DIRS 175927], } \\
\text { ACC: MOL.19950327.0062; } \\
\text { GS931208314211.046 } \\
\text { [DIRS 150012], } \\
\text { ACC: NNA.19940608.0247 }\end{array}$ & Qualified and Verified \\
\hline $\begin{array}{l}\text { USW UZ- } \\
\text { N64 }\end{array}$ & 765728.12 & 559435.53 & MO9906GPS98410.000 [DIRS 109059] ${ }^{a}$ & 1.80 & 0.55 & $\begin{array}{l}\text { GS940308314211.016 } \\
\text { [DIRS 146474], } \\
\text { ACC: MOL.19941101.0065 }\end{array}$ & Qualified and Verified \\
\hline $\begin{array}{l}\text { UE-25 } \\
\text { NRG\#2 }\end{array}$ & 765763.71 & 569162.45 & MO9906GPS98410.000 [DIRS 109059] & 0.00 & 0.00 & $\begin{array}{l}\text { GS931008314211.039 } \\
\text { [DIRS 150009], } \\
\text { ACC: NNA.19940105.0085 }\end{array}$ & Qualified and Verified \\
\hline $\begin{array}{l}\text { UE-25 } \\
\text { NRG\#3 }\end{array}$ & 766250.60 & 568316.10 & MO9906GPS98410.000 [DIRS 109059] & 0.00 & 0.00 & $\begin{array}{l}\text { GS931008314211.037 } \\
\text { [DIRS 150007], } \\
\text { ACC: NNA.19940105.0083; } \\
\text { SNF29041993002.038 } \\
\text { [DIRS 175958], } \\
\text { ACC: MOL.19950327.0079 }\end{array}$ & Qualified and Verified \\
\hline $\begin{array}{l}\text { USW } \\
\text { NRG-6 }\end{array}$ & 766726.28 & 564187.17 & MO0506GSC05047.000 [DIRS 175919] & 0.00 & 0.00 & $\begin{array}{l}\text { GS931008314211.045 } \\
\text { [DIRS 150010], } \\
\text { ACC: NNA.19940608.0248 }\end{array}$ & Qualified and verified \\
\hline $\begin{array}{l}\text { UE-25 } \\
\text { NRG\#4 }\end{array}$ & 767080.21 & 566819.99 & MO9906GPS98410.000 [DIRS 109059] & 0.00 & 0.00 & $\begin{array}{l}\text { SNF29041993002.039 } \\
\text { [DIRS 175959], } \\
\text { ACC: MOL.19950327.0083 }\end{array}$ & Qualified and Verified \\
\hline $\begin{array}{l}\text { USW UZ- } \\
\text { N37 }\end{array}$ & 767498.87 & 563713.51 & MO0506GSC05047.000 [DIRS 175919] & 36.30 & 11.06 & $\begin{array}{l}\text { GS940208314211.005 } \\
\text { [DIRS 152563] }^{9}\end{array}$ & Qualified and Verified \\
\hline $\begin{array}{l}\text { UE-25 } \\
\text { NRG\#5 }\end{array}$ & 767889.61 & 564769.87 & MO9906GPS98410.000 [DIRS 109059] ${ }^{\mathrm{a}}$ & 0.00 & 0.00 & $\begin{array}{l}\text { SNF29041993002.040 } \\
\text { [DIRS 175960], } \\
\text { MOL.19950327.0087 }\end{array}$ & Qualified and Verified \\
\hline $\begin{array}{l}\text { UE-25 } \\
\text { UZN\#63 }\end{array}$ & 768837.28 & 566169.64 & MO9906GPS98410.000 [DIRS 109059] ${ }^{\mathrm{a}}$ & 8.60 & 2.62 & $\begin{array}{l}\text { GS940308314211.017 } \\
\text { [DIRS 155534], } \\
\text { ACC: MOL.19941101.0064 }\end{array}$ & Qualified and Verified \\
\hline $\begin{array}{l}\text { USW } \\
\text { NRG-7a }\end{array}$ & 768880.11 & 562983.99 & MO9906GPS98410.000 [DIRS 109059] & 17.00 & 5.18 & $\begin{array}{l}\text { SNF29041993002.042 } \\
\text { [DIRS 166437], } \\
\text { Table S97096_001 }\end{array}$ & Qualified and Verified \\
\hline
\end{tabular}


Table A-1. Boreholes and Surficial Deposit Depth Measurements (Continued)

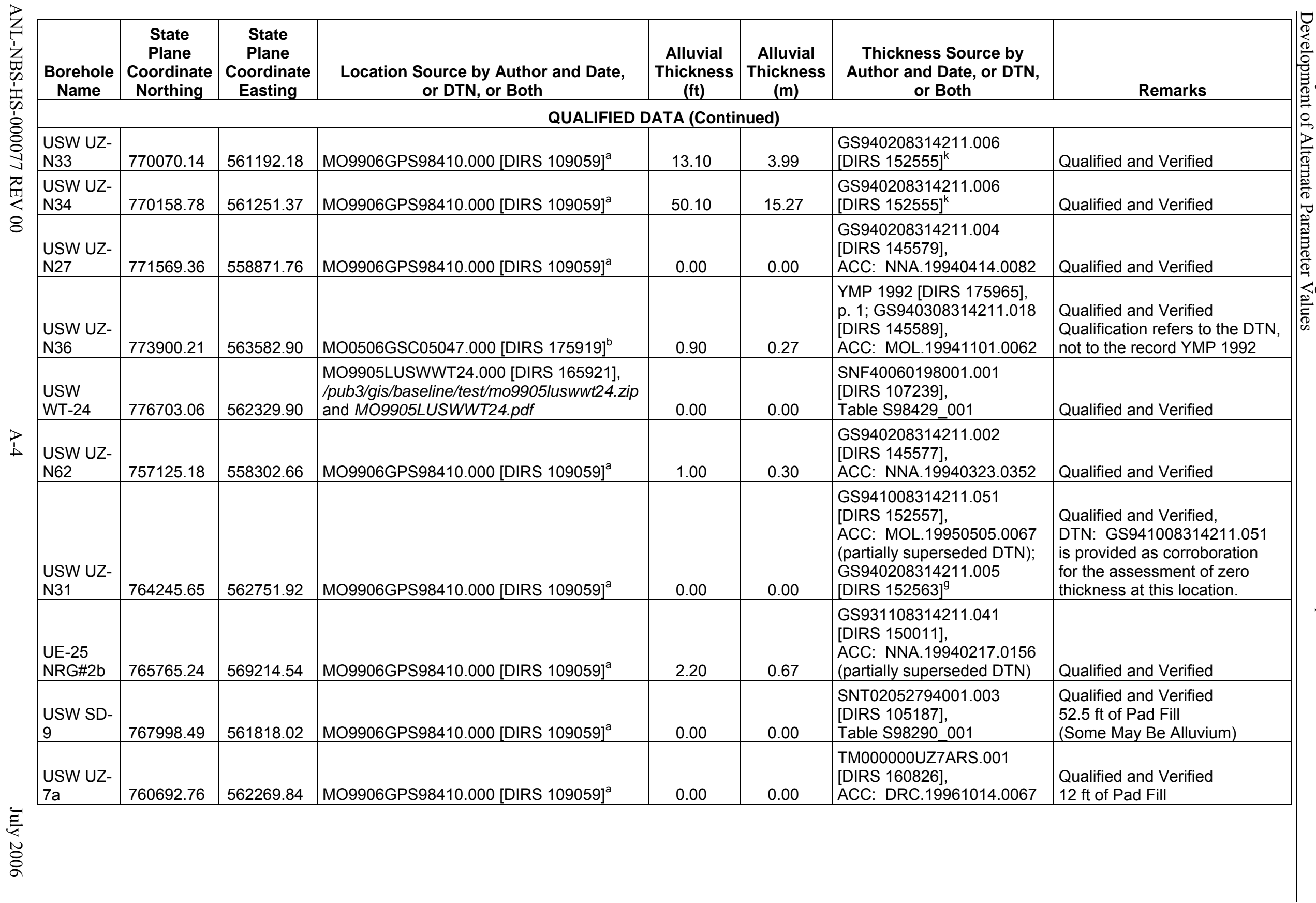


Table A-1. Boreholes and Surficial Deposit Depth Measurements (Continued)

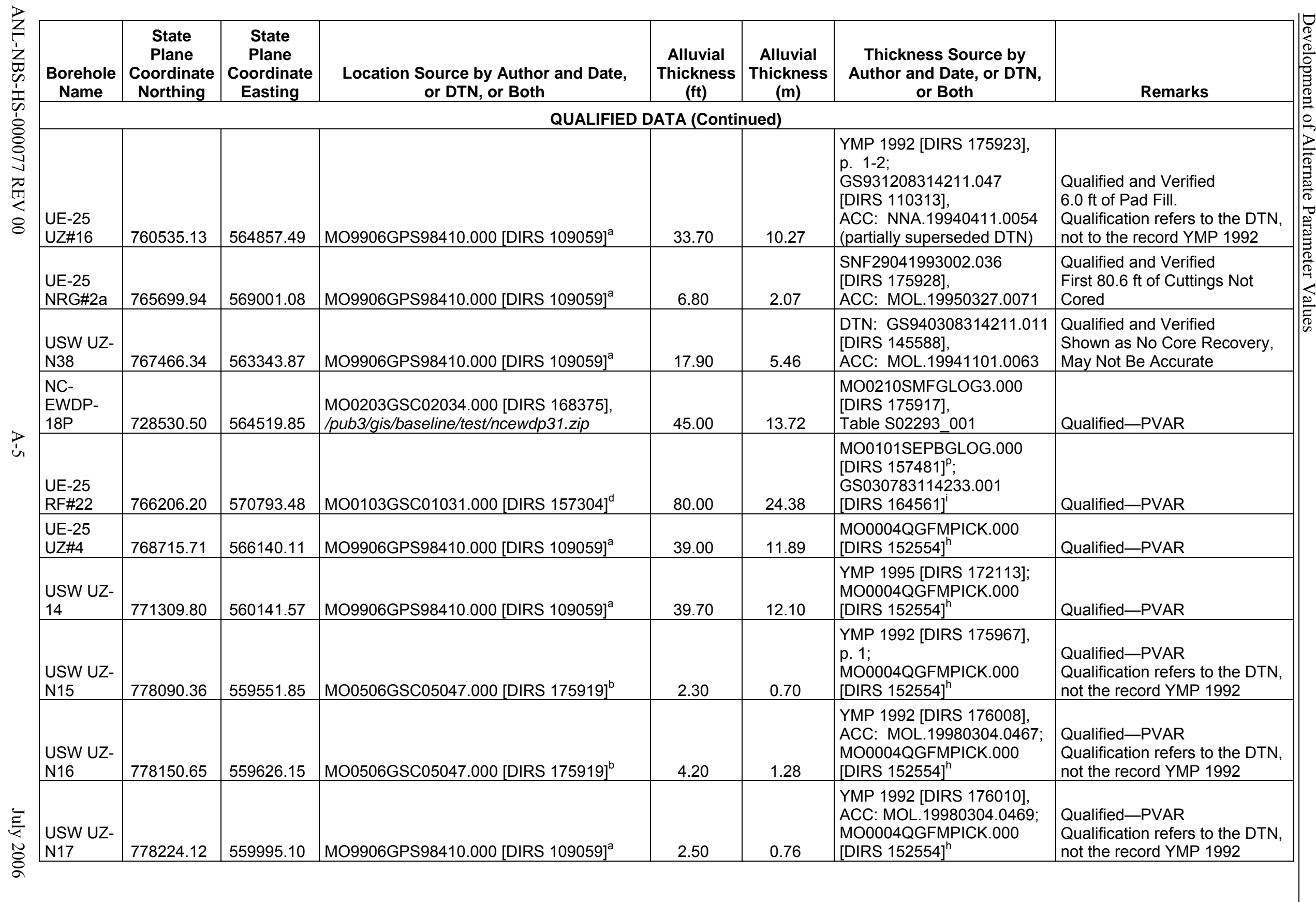


Table A-1. Boreholes and Surficial Deposit Depth Measurements (Continued)

\begin{tabular}{|c|c|c|c|c|c|c|c|}
\hline $\begin{array}{c}\text { Borehole } \\
\text { Name }\end{array}$ & \begin{tabular}{|c|} 
State \\
Plane \\
Coordinate \\
Northing \\
\end{tabular} & \begin{tabular}{|c|} 
State \\
Plane \\
Coordinate \\
Easting \\
\end{tabular} & $\begin{array}{l}\text { Location Source by Author and Date, } \\
\text { or DTN, or Both }\end{array}$ & $\begin{array}{c}\text { Alluvial } \\
\text { Thickness } \\
\text { (ft) }\end{array}$ & $\begin{array}{c}\text { Alluvial } \\
\text { Thickness } \\
\text { (m) }\end{array}$ & $\begin{array}{c}\text { Thickness Source by } \\
\text { Author and Date, or DTN, } \\
\text { or Both }\end{array}$ & Remarks \\
\hline \multicolumn{8}{|c|}{ QUALIFIED DATA (Continued) } \\
\hline $\begin{array}{l}\text { USW } \\
\text { UZ-N11 }\end{array}$ & 780573.74 & 559020.91 & MO0506GSC05047.000 [DIRS 175919] ${ }^{\mathrm{b}}$ & 1.70 & 0.52 & $\begin{array}{l}\text { Gertz } 1992 \text { [DIRS 175968]; } \\
\text { MO0004QGFMPICK.000 } \\
\text { [DIRS 152554] }^{\mathrm{h}}\end{array}$ & $\begin{array}{l}\text { Qualified-PVAR } \\
\text { Qualification refers to the DTN, } \\
\text { not the record Gertz } 1992\end{array}$ \\
\hline $\begin{array}{l}\text { UE-25 } \\
\text { RF\#14 }\end{array}$ & 765308.73 & 571065.44 & MO0103GSC01031.000 [DIRS 157304] ${ }^{\mathrm{d}}$ & 101.80 & 31.03 & $\begin{array}{l}\text { MO0101SEPBGLOG.000 } \\
\text { [DIRS 157481] } \\
\text { GS030783114233.001 } \\
\text { [DIRS 164561] }^{\mathrm{i}}\end{array}$ & Qualified-PVAR \\
\hline $\begin{array}{l}\text { UE-25 } \\
\text { RF\#19 }\end{array}$ & 765880.41 & 571383.73 & MO0103GSC01031.000 [DIRS 157304] ${ }^{\mathrm{d}}$ & 120.00 & 36.58 & $\begin{array}{l}\text { GS030783114233.001 } \\
\text { [DIRS 164561] }^{\mathrm{i}}\end{array}$ & Qualified-PVAR \\
\hline $\begin{array}{l}\text { UE-25 } \\
\text { RF\#29 }\end{array}$ & 766018.46 & 570836.04 & MO0103GSC01031.000 [DIRS 157304] ${ }^{d}$ & 85.00 & 25.91 & $\begin{array}{l}\text { MO0101SEPBGLOG.000 } \\
\text { [DIRS 157481]; } \\
\text { GS030783114233.001 } \\
\text { [DIRS 164561] }^{\mathrm{p}}\end{array}$ & Qualified-PVAR \\
\hline $\begin{array}{l}\text { UE-25 } \\
\text { RF\#17 }\end{array}$ & 766075.86 & 571041.88 & MO0103GSC01031.000 [DIRS 157304] ${ }^{d}$ & 92.40 & 28.16 & $\begin{array}{l}\text { MO0101SEPBGLOG.000 } \\
\text { [DIRS 157481] }^{p} \\
\text { GS030783114233.001 } \\
\text { [DIRS 164561] }^{\text {i }}\end{array}$ & Qualified-PVAR \\
\hline USW G-1 & 770500.20 & 561000.50 & MO0106STRATHFM.030 [DIRS 155591] ${ }^{q}$ & 60.00 & 18.29 & $\begin{array}{l}\text { MO0106STRATHFM.030 } \\
\text { [DIRS 155591] }^{q}\end{array}$ & Qualified-PVAR \\
\hline USW G-2 & 778822.20 & 560502.00 & MO0106STRATHFM.031 [DIRS 155592] & 0.00 & 0.00 & $\begin{array}{l}\text { MO0106STRATHFM.031 } \\
\text { [DIRS 155592]' }\end{array}$ & Qualified-PVAR \\
\hline USW G-3 & 752780.00 & 558483.00 & MO0106STRATHFM.004 [DIRS 155539] ${ }^{\mathrm{m}}$ & 0.00 & 0.00 & $\begin{array}{l}\text { MO0106STRATHFM.004 } \\
\text { [DIRS 155539] }^{\mathrm{m}}\end{array}$ & Qualified-PVAR \\
\hline USW H-1 & 770254.00 & 562388.00 & MO0106STRATHFM.028 [DIRS 155589] ${ }^{\mathrm{n}}$ & 0.00 & 0.00 & $\begin{array}{l}\text { MO0106STRATHFM.028 } \\
\text { [DIRS 155589] }^{\mathrm{n}}\end{array}$ & Qualified-PVAR \\
\hline USW G-4 & 765807.51 & 563081.78 & MO9906GPS98410.000 [DIRS 109059] ${ }^{\mathrm{a}}$ & 30.00 & 9.14 & $\begin{array}{l}\text { MO0004QGFMPICK.000 } \\
\text { [DIRS 152554] }^{\mathrm{h}}\end{array}$ & Qualified-PVAR \\
\hline $\begin{array}{l}\text { UE-25 } \\
\text { J\#13 }\end{array}$ & 749201.99 & 579647.91 & MO9906GPS98410.000 [DIRS 109059] ${ }^{\mathrm{a}}$ & 435.00 & 132.59 & $\begin{array}{l}\text { MO0004QGFMPICK.000 } \\
\text { [DIRS 152554] }^{\mathrm{h}}\end{array}$ & Qualified-PVAR \\
\hline
\end{tabular}


Table A-1. Boreholes and Surficial Deposit Depth Measurements (Continued)

\begin{tabular}{|c|c|c|c|c|c|c|c|}
\hline $\begin{array}{l}\text { Borehole } \\
\text { Name }\end{array}$ & $\begin{array}{c}\text { State } \\
\text { Plane } \\
\text { Coordinate } \\
\text { Northing }\end{array}$ & $\begin{array}{l}\text { State } \\
\text { Plane } \\
\text { Coordinate } \\
\text { Easting }\end{array}$ & $\begin{array}{c}\text { Location Source by Author and Date, } \\
\text { or DTN, or Both }\end{array}$ & $\begin{array}{l}\text { Alluvial } \\
\text { Thickness } \\
\text { (ft) }\end{array}$ & $\begin{array}{c}\text { Alluvial } \\
\text { Thickness } \\
\text { (m) }\end{array}$ & $\begin{array}{c}\text { Thickness Source by } \\
\text { Author and Date, or DTN, } \\
\text { or Both }\end{array}$ & Remarks \\
\hline \multicolumn{8}{|c|}{ QUALIFIED DATA (Continued) } \\
\hline USW a\#1 & 764900.99 & 566349.89 & MO9906GPS98410.000 [DIRS 109059] ${ }^{a}$ & 30.00 & 9.14 & $\begin{array}{l}\text { MO0004QGFMPICK.000 } \\
\text { [DIRS 152554] }^{\mathrm{h}}\end{array}$ & Qualified-PVAR \\
\hline $\begin{array}{l}\text { UE-25 } \\
\text { a\#4 }\end{array}$ & 767972.39 & 564471.85 & MO9906GPS98410.000 [DIRS 109059] & 30.00 & 9.14 & $\begin{array}{l}\text { MO0004QGFMPICK.000 } \\
\text { [DIRS 152554] }^{\mathrm{h}}\end{array}$ & Qualified-PVAR \\
\hline $\begin{array}{l}\text { UE-25 } \\
\text { a\#5 }\end{array}$ & 766956.42 & 564755.20 & MO9906GPS98410.000 [DIRS 109059] & 90.00 & 27.43 & $\begin{array}{l}\text { MO0004QGFMPICK.000 } \\
\text { [DIRS 152554] }^{\mathrm{h}}\end{array}$ & Qualified-PVAR \\
\hline $\begin{array}{l}\text { UE-25 } \\
\text { a\#6 }\end{array}$ & 765899.90 & 564500.89 & MO9906GPS98410.000 [DIRS 109059] & 20.00 & 6.10 & $\begin{array}{l}\text { MO0004QGFMPICK.000 } \\
\text { [DIRS 152554] }^{\mathrm{h}}\end{array}$ & Qualified-PVAR \\
\hline $\begin{array}{l}\text { UE-25 } \\
\text { a\#7 }\end{array}$ & 766250.03 & 565468.31 & MO9906GPS98410.000 [DIRS 109059] & 165.00 & 50.29 & $\begin{array}{l}\text { MO0004QGFMPICK.000 } \\
\text { [DIRS 152554] }^{\mathrm{h}}\end{array}$ & Qualified-PVAR \\
\hline $\begin{array}{l}\text { UE-25 } \\
\mathrm{b \# 1}\end{array}$ & 765244.31 & 566416.20 & MO9906GPS98410.000 [DIRS 109059] & 156.00 & 47.55 & $\begin{array}{l}\text { MO0004QGFMPICK.000 } \\
\text { [DIRS 152554] }^{\mathrm{h}}\end{array}$ & Qualified-PVAR \\
\hline $\begin{array}{l}\text { UE-25 } \\
\text { C\#1 }\end{array}$ & 757096.78 & 569680.57 & MO9906GPS98410.000 [DIRS 109059] & 0.00 & 0.00 & $\begin{array}{l}\text { MO0004QGFMPICK.000 } \\
\text { [DIRS 152554] }^{\mathrm{h}}\end{array}$ & Qualified-PVAR \\
\hline $\begin{array}{l}\text { UE-25 } \\
\text { C\#2 }\end{array}$ & 756849.64 & 569633.94 & MO9906GPS98410.000 [DIRS 109059] & 69.90 & 21.31 & $\begin{array}{l}\text { MO0004QGFMPICK.000 } \\
\text { [DIRS 152554] }^{\mathrm{h}}\end{array}$ & Qualified_-PVAR \\
\hline $\begin{array}{l}\text { UE-25 } \\
\text { C\#3 }\end{array}$ & 756910.88 & 569554.93 & MO9906GPS98410.000 [DIRS 109059] & 80.10 & 24.41 & $\begin{array}{l}\text { MO0004QGFMPICK.000 } \\
\text { [DIRS 152554] }^{\mathrm{h}}\end{array}$ & Qualified_PVAR \\
\hline USW H-3 & 756542.01 & 558451.90 & MO9906GPS98410.000 [DIRS 109059] & 0.00 & 0.00 & $\begin{array}{l}\text { MO0004QGFMPICK.000 } \\
\text { [DIRS 152554] }\end{array}$ & Qualified_-PVAR \\
\hline USW H-4 & 761644.48 & 563911.40 & MO9906GPS98410.000 [DIRS 109059] ${ }^{\mathrm{a}}$ & 0.00 & 0.00 & $\begin{array}{l}\text { MO0004QGFMPICK.000 } \\
\text { [DIRS 152554] }^{\mathrm{h}}\end{array}$ & Qualified-PVAR \\
\hline USW H-5 & 766634.12 & 558908.41 & MO9906GPS98410.000 [DIRS 109059] ${ }^{\mathrm{a}}$ & 0.00 & 0.00 & $\begin{array}{l}\text { MO0004QGFMPICK.000 } \\
\text { [DIRS 152554] }^{\mathrm{h}}\end{array}$ & Qualified_-PVAR \\
\hline USW H-6 & 763299.43 & 554074.96 & MO9906GPS98410.000 [DIRS 109059] & 29.90 & 9.11 & $\begin{array}{l}\text { MO0004QGFMPICK.000 } \\
\text { [DIRS 152554] }^{\mathrm{h}}\end{array}$ & Qualified_-PVAR \\
\hline $\begin{array}{l}\text { USW UZ- } \\
1\end{array}$ & 771277.35 & 560221.57 & MO9906GPS98410.000 [DIRS 109059] & 40.00 & 12.19 & $\begin{array}{l}\text { MO0004QGFMPICK.000 } \\
\text { [DIRS 152554] }^{\mathrm{h}}\end{array}$ & Qualified_-PVAR \\
\hline $\begin{array}{l}\text { USW } \\
\text { UZ-6 }\end{array}$ & 759730.18 & 558324.98 & MO9906GPS98410.000 [DIRS 109059] & 0.00 & 0.00 & $\begin{array}{l}\text { MO0004QGFMPICK.000 } \\
\text { [DIRS 152554] }^{\mathrm{h}}\end{array}$ & Qualified_-PVAR \\
\hline $\begin{array}{l}\text { UE-25 } \\
\text { UZ\#5 }\end{array}$ & 768593.15 & 566136.13 & MO9906GPS98410.000 [DIRS 109059] ${ }^{\mathrm{a}}$ & 3.00 & 0.91 & $\begin{array}{l}\text { MO0004QGFMPICK.000 } \\
\text { [DIRS 152554] }^{\mathrm{h}}\end{array}$ & Qualified-PVAR \\
\hline $\begin{array}{l}\text { USW } \\
\text { WT-1 }\end{array}$ & 753942.15 & 563739.20 & MO9906GPS98410.000 [DIRS 109059] & 30.00 & 9.14 & $\begin{array}{l}\text { MO0004QGFMPICK.000 } \\
\text { [DIRS 152554] }^{\mathrm{h}}\end{array}$ & Qualified_-PVAR \\
\hline
\end{tabular}


Table A-1. Boreholes and Surficial Deposit Depth Measurements (Continued)

\begin{tabular}{|c|c|c|c|c|c|c|}
\hline $\begin{array}{l}\text { State } \\
\text { Plane } \\
\text { Coordinate } \\
\text { Northing } \\
\end{array}$ & $\begin{array}{c}\text { State } \\
\text { Plane } \\
\text { Coordinate } \\
\text { Easting } \\
\end{array}$ & $\begin{array}{l}\text { Location Source by Author and Date, } \\
\text { or DTN, or Both }\end{array}$ & $\begin{array}{c}\text { Alluvial } \\
\text { Thickness } \\
\text { (ft) }\end{array}$ & \begin{tabular}{|c} 
Alluvial \\
Thickness \\
$(\mathrm{m})$
\end{tabular} & $\begin{array}{c}\text { Thickness Source by } \\
\text { Author and Date, or DTN, } \\
\text { or Both }\end{array}$ & Remarks \\
\hline \multicolumn{7}{|c|}{$\begin{array}{l}\text { QUALIFIED DATA (Continued) } \\
\end{array}$} \\
\hline 60661.55 & 561924.01 & MO9906GPS98410.000 [DIRS 109059] $]^{\mathrm{a}}$ & 60.00 & 18.29 & $\begin{array}{l}\text { MO0004QGFMPICK.000 } \\
\text { [DIRS 152554] }^{\mathrm{h}}\end{array}$ & Qualified_PVAR \\
\hline 45995.54 & 573385.20 & MO9906GPS98410.000 [DIRS 109059] $]^{\mathrm{a}}$ & 11.00 & 3.35 & $\begin{array}{l}\text { MO0004QGFMPICK.000 } \\
\text { [DIRS 152554] }^{\mathrm{h}}\end{array}$ & Qualified_PVAR \\
\hline 68511.94 & 568038.29 & MO9906GPS98410.000 [DIRS 109059] ${ }^{\mathrm{a}}$ & 51.00 & 15.54 & $\begin{array}{l}\text { MO0004QGFMPICK.000 } \\
\text { [DIRS 152554] }^{\mathrm{h}}\end{array}$ & Qualified_PVAR \\
\hline 80576.15 & 564523.62 & MO9906GPS98410.000 [DIRS 109059] ${ }^{\mathrm{a}}$ & 170.00 & 51.82 & $\begin{array}{l}\text { MO0004QGFMPICK.000 } \\
\text { [DIRS 152554] }^{\mathrm{h}}\end{array}$ & Qualified_PVAR \\
\hline 55570.55 & 553891.65 & MO9906GPS98410.000 [DIRS 109059] $]^{\mathrm{a}}$ & 40.00 & 12.19 & $\begin{array}{l}\text { MO0004QGFMPICK.000 } \\
\text { [DIRS 152554] }^{\mathrm{h}}\end{array}$ & Qualified_PVAR \\
\hline 48771.58 & 553302.33 & MO9906GPS98410.000 [DIRS 109059] $]^{a}$ & 60.00 & 18.29 & $\begin{array}{l}\text { MO0004QGFMPICK.000 } \\
\text { [DIRS 152554] }^{\mathrm{h}}\end{array}$ & Qualified_PVAR \\
\hline 39071.30 & 558376.70 & MO9906GPS98410.000 [DIRS 109059] ${ }^{\mathrm{a}}$ & 40.00 & 12.19 & $\begin{array}{l}\text { MO0004QGFMPICK.000 } \\
\text { [DIRS 152554] }^{\mathrm{h}}\end{array}$ & Qualified_PVAR \\
\hline 39726.71 & 567011.79 & MO9906GPS98410.000 [DIRS 109059] $]^{\mathrm{a}}$ & 60.00 & 18.29 & $\begin{array}{l}\text { MO0004QGFMPICK.000 } \\
\text { [DIRS 152554] }^{\mathrm{h}}\end{array}$ & Qualified_PVAR \\
\hline 56885.20 & 578842.27 & MO9906GPS98410.000 [DIRS 109059] $]^{\mathrm{a}}$ & 220.00 & 67.06 & $\begin{array}{l}\text { MO0004QGFMPICK.000 } \\
\text { [DIRS 152554] }{ }^{\mathrm{h}}\end{array}$ & Qualified_PVAR \\
\hline 61651.39 & 575210.21 & MO9906GPS98410.000 [DIRS 109059] ${ }^{\mathrm{a}}$ & 107.00 & 32.61 & $\begin{array}{l}\text { MO0004QGFMPICK.000 } \\
\text { [DIRS 152554] }\end{array}$ & Qualified_PVAR \\
\hline 66116.98 & 579806.27 & MO9906GPS98410.000 [DIRS 109059] ${ }^{a}$ & 210.00 & 64.01 & $\begin{array}{l}\text { MO0004QGFMPICK.000 } \\
\text { [DIRS 152554] }^{\mathrm{h}}\end{array}$ & Qualified_PVAR \\
\hline 74420.20 & 570395.13 & MO9906GPS98410.000 [DIRS 109059] ${ }^{\mathrm{a}}$ & 137.00 & 41.76 & $\begin{array}{l}\text { MO0004QGFMPICK.000 } \\
\text { [DIRS 152554] }^{\mathrm{h}}\end{array}$ & Qualified_PVAR \\
\hline 48421.52 & 566212.01 & MO9906GPS98410.000 [DIRS 109059] ${ }^{\mathrm{a}}$ & 30.00 & 9.14 & $\begin{array}{l}\text { MO0004QGFMPICK.000 } \\
\text { [DIRS 152554] }^{\mathrm{h}}\end{array}$ & Qualified_PVAR \\
\hline 71167.22 & 564854.65 & MO9906GPS98410.000 [DIRS 109059] ${ }^{\mathrm{a}}$ & 0.00 & 0.00 & $\begin{array}{l}\text { MO0004QGFMPICK.000 } \\
\text { [DIRS 152554] }^{\mathrm{h}}\end{array}$ & Qualified_PVAR \\
\hline 65968.15 & 570626.38 & MO0103GSC01031.000 [DIRS 157304] ${ }^{\mathrm{d}}$ & 60.00 & 18.29 & $\begin{array}{l}\text { GS030783114233.001 } \\
\text { [DIRS 164561] }^{\mathrm{i}}\end{array}$ & $\begin{array}{l}\text { Qualified_PVAR } \\
10 \mathrm{ft} \text { of Pad Fill }\end{array}$ \\
\hline 65311.43 & 570465.03 & MO0103GSC01031.000 [DIRS 157304] ${ }^{\mathrm{d}}$ & 64.00 & 19.51 & $\begin{array}{l}\text { GS030783114233.001 } \\
\text { [DIRS 164561] }\end{array}$ & $\begin{array}{l}\text { Qualified_PVAR } \\
12 \mathrm{ft} \text { of Pad Fill }\end{array}$ \\
\hline
\end{tabular}


Table A-1. Boreholes and Surficial Deposit Depth Measurements (Continued)

\begin{tabular}{|c|c|c|c|c|c|c|c|}
\hline $\begin{array}{l}\text { Borehole } \\
\text { Name }\end{array}$ & \begin{tabular}{|c|} 
State \\
Plane \\
Coordinate \\
Northing \\
\end{tabular} & \begin{tabular}{|c|} 
State \\
Plane \\
Coordinate \\
Easting \\
\end{tabular} & $\begin{array}{l}\text { Location Source by Author and Date, } \\
\text { or DTN, or Both }\end{array}$ & \begin{tabular}{|c} 
Alluvial \\
Thickness \\
(ft)
\end{tabular} & \begin{tabular}{|c} 
Alluvial \\
$\begin{array}{c}\text { Thickness } \\
\text { (m) }\end{array}$ \\
\end{tabular} & $\begin{array}{c}\text { Thickness Source by } \\
\text { Author and Date, or DTN, } \\
\text { or Both }\end{array}$ & Remarks \\
\hline \multicolumn{8}{|c|}{ QUALIFIED DATA (Continued) } \\
\hline $\begin{array}{l}\text { UE-25 } \\
\text { RF\#13 }\end{array}$ & 765500.04 & 570720.12 & $\begin{array}{l}\text { MO9902ASBUBOLC.000 [DIRS 103793], } \\
\text { /pub3/gis/baseline/test/mo9902asbubolc.zip }\end{array}$ & 85.50 & 26.06 & $\begin{array}{l}\text { GS030783114233.001 } \\
\text { [DIRS 164561]; } \\
\text { MO9903BLOGSOIL.000 } \\
\text { [DIRS 103790], } \\
\text { Table S99142_001 } \\
\end{array}$ & $\begin{array}{l}\text { Qualified-PVAR } \\
12.5 \mathrm{ft} \text { of Pad Fill } \\
\end{array}$ \\
\hline $\begin{array}{l}\text { UE-25 } \\
\text { RF\#26 }\end{array}$ & 765247.95 & 570579.71 & MO0103GSC01031.000 [DIRS 157304] ${ }^{d}$ & 71.00 & 21.64 & $\begin{array}{l}\text { GS030783114233.001 } \\
\text { [DIRS 164561] }^{i}\end{array}$ & $\begin{array}{l}\text { Qualified-PVAR } \\
14 \mathrm{ft} \text { of Pad Fill }\end{array}$ \\
\hline $\begin{array}{l}\text { UE-25 } \\
\text { RF\#16 }\end{array}$ & 765055.54 & 570472.65 & MO0103GSC01031.000 [DIRS 157304] ${ }^{\mathrm{d}}$ & 53.30 & 16.25 & $\begin{array}{l}\text { MO0101SEPBGLOG.000 } \\
\text { [DIRS 157481] } \\
\text { GS030783114233.001 } \\
\text { [DIRS 164561] }^{\mathrm{i}}\end{array}$ & $\begin{array}{l}\text { Qualified-PVAR } \\
22.4 \mathrm{ft} \text { of Pad Fill } \\
\end{array}$ \\
\hline $\begin{array}{l}\text { UE-25 } \\
\text { RF\#20 }\end{array}$ & 765637.36 & 570796.82 & MO0103GSC01031.000 [DIRS 157304] ${ }^{\mathrm{d}}$ & 70.00 & 21.34 & $\begin{array}{l}\text { MO0101SEPBGLOG.000 } \\
\text { [DIRS 157481] } \\
\text { GS030783114233.001 } \\
\text { [DIRS 164561] }\end{array}$ & $\begin{array}{l}\text { Qualified-PVAR } \\
28 \mathrm{ft} \text { of Pad Fill }\end{array}$ \\
\hline $\begin{array}{l}\text { UE-25 } \\
\text { RF\#28 }\end{array}$ & 765510.21 & 570104.87 & MO0103GSC01031.000 [DIRS 157304] ${ }^{\mathrm{d}}$ & 10.00 & 3.05 & $\begin{array}{l}\text { MO0101SEPBGLOG.000 } \\
\text { [DIRS 157481] }^{\mathrm{p}} \\
\text { GS030783114233.001 } \\
\text { [DIRS 164561] }^{\mathrm{i}}\end{array}$ & $\begin{array}{l}\text { Qualified-PVAR } \\
5 \mathrm{ft} \text { of Pad Fill }\end{array}$ \\
\hline $\begin{array}{l}\text { UE-25 } \\
\text { RF\#21 }\end{array}$ & 765898.91 & 570739.18 & MO0103GSC01031.000 [DIRS 157304] ${ }^{\mathrm{d}}$ & 110.00 & 33.53 & $\begin{array}{l}\text { GS030783114233.001 } \\
\text { [DIRS 164561] }^{\mathrm{i}}\end{array}$ & $\begin{array}{l}\text { Qualified-PVAR } \\
5 \mathrm{ft} \text { of Pad Fill } \\
\end{array}$ \\
\hline $\begin{array}{l}\text { UE-25 } \\
\text { RF\#15 }\end{array}$ & 765773.64 & 570224.85 & MO0103GSC01031.000 [DIRS 157304] ${ }^{\mathrm{d}}$ & 0.00 & 0.00 & $\begin{array}{l}\text { GS030783114233.001 } \\
\text { [DIRS 164561] }^{i}\end{array}$ & $\begin{array}{l}\text { Qualified-PVAR } \\
5 \mathrm{ft} \text { of Pad Fill }\end{array}$ \\
\hline $\begin{array}{l}\text { UE-25 } \\
\text { RF\#24 }\end{array}$ & 766344.31 & 570542.26 & MO0103GSC01031.000 [DIRS 157304] ${ }^{d}$ & 20.00 & 6.10 & $\begin{array}{l}\text { MO0101SEPBGLOG.000 } \\
\text { [DIRS 157481] }^{\text {p }} \\
\text { GS030783114233.001 } \\
\text { [DIRS 164561] }^{i}\end{array}$ & $\begin{array}{l}\text { Qualified-PVAR } \\
10 \mathrm{ft} \text { of Pad Fill }\end{array}$ \\
\hline \multicolumn{8}{|c|}{ UNQUALIFIED DATA } \\
\hline $\begin{array}{l}\text { USW } \\
\text { SD-12 }\end{array}$ & 761956.56 & 561605.61 & MO9906GPS98410.000 [DIRS 109059] ${ }^{a}$ & 0.00 & 0.00 & $\begin{array}{l}\text { TM000000SD12RS.014 } \\
\text { [DIRS 175924], } \\
\text { ACC: DRC.19960926.0174; } \\
\text { GS000308314211.002 } \\
\text { [DIRS 158000], } \\
\text { Table S00274_001 }\end{array}$ & $\begin{array}{l}\text { Location is Qualified and } \\
\text { Verified; } \\
\text { Soil Depth in DIRS } 175924 \text { is } \\
\text { Qualified and Not Verified; } \\
\text { Soil Depth in DIRS } 158000 \text { is } \\
\text { not qualified. } \\
12.9 \mathrm{ft} \text { of Pad Fill }\end{array}$ \\
\hline
\end{tabular}


Table A-1. Boreholes and Surficial Deposit Depth Measurements (Continued)

\begin{tabular}{|c|c|c|c|c|c|c|c|}
\hline $\begin{array}{c}\text { Borehole } \\
\text { Name }\end{array}$ & \begin{tabular}{|c|} 
State \\
Plane \\
Coordinate \\
Northing \\
\end{tabular} & $\begin{array}{c}\text { State } \\
\text { Plane } \\
\text { Coordinate } \\
\text { Easting } \\
\end{array}$ & $\begin{array}{l}\text { Location Source by Author and Date, } \\
\text { or DTN, or Both }\end{array}$ & $\begin{array}{c}\text { Alluvial } \\
\text { Thickness } \\
\text { (ft) }\end{array}$ & $\begin{array}{c}\text { Alluvial } \\
\text { Thickness } \\
\text { (m) }\end{array}$ & $\begin{array}{c}\text { Thickness Source by } \\
\text { Author and Date, or DTN, } \\
\text { or Both }\end{array}$ & Remarks \\
\hline \multicolumn{8}{|c|}{ UNQUALIFIED DATA (Continued) } \\
\hline $\begin{array}{l}\text { USW } \\
\text { NRG-7 }\end{array}$ & 768846.23 & 563004.86 & $\begin{array}{l}\text { YMP } 1993 \text { [DIRS 175962], p. 22C - } \\
\text { Proposed Location NRG-7 }\end{array}$ & 0.00 & 0.00 & $\begin{array}{l}\text { YMP } 1993 \text { [DIRS 175963], } \\
\text { p. } 1\end{array}$ & Unqualified \\
\hline $\begin{array}{l}\text { UE-25 } \\
\text { NRG\#2c }\end{array}$ & 765771.68 & 569189.78 & MO9906GPS98410.000 [DIRS 109059] & $\begin{array}{c}\text { Not } \\
\text { Determined } \\
\end{array}$ & - & $\begin{array}{l}\text { YMP } 1994 \text { [DIRS 175930], } \\
\text { ACC: MOL.19940719.0031 }\end{array}$ & $\begin{array}{l}\text { Location is Qualified and } \\
\text { Verified; } \\
\text { Soil Depth is Unqualified - } \\
\text { Alluvial Section Not Logged }\end{array}$ \\
\hline $\begin{array}{l}\text { UE-25 } \\
\text { NRG\#2d }\end{array}$ & 765825.10 & 569132.29 & MO9906GPS98410.000 [DIRS 109059] ${ }^{a}$ & $\begin{array}{c}\text { Not } \\
\text { Determined }\end{array}$ & - & 一 & $\begin{array}{l}\text { Location is Qualified and } \\
\text { Verified; } \\
\text { Soil Depth is Unqualified - } \\
\text { Log Not Available for } \\
\text { Alluvial Section }\end{array}$ \\
\hline $\begin{array}{l}\text { UE-25 } \\
\text { J\#12 }\end{array}$ & 733508.28 & 581012.07 & MO9906GPS98410.000 [DIRS 109059] & 515.00 & 156.97 & $\begin{array}{l}\text { Dixon } 1992 \text { [DIRS 175970], } \\
\text { ACC: MOL.19980218.0561, } \\
\text { p. } 24\end{array}$ & $\begin{array}{l}\text { Location is Qualified and } \\
\text { Verified; } \\
\text { Soil Depth is Unqualified }\end{array}$ \\
\hline $\begin{array}{l}\text { UE-25 } \\
\text { JF\#3 }\end{array}$ & 730875.40 & 581179.42 & MO9906GPS98410.000 [DIRS 109059] ${ }^{\mathrm{a}}$ & 487.00 & 148.44 & $\begin{array}{l}\text { TM0000000000CL.005 } \\
\text { [DIRS 175971], } \\
\text { ACC: MOL.20011018.0232 }\end{array}$ & $\begin{array}{l}\text { Location is Qualified and } \\
\text { Verified; } \\
\text { Soil Depth is Unqualified }\end{array}$ \\
\hline $\begin{array}{l}\text { USW } \\
\text { UZ-N40 }\end{array}$ & 766176.22 & 564221.41 & MO9906GPS98410.000 [DIRS 109059] & 2.89 & 0.88 & $\begin{array}{l}\text { GS910808312212.001 } \\
\text { [DIRS 175972] }^{\dagger}\end{array}$ & $\begin{array}{l}\text { Location is Qualified and } \\
\text { Verified; } \\
\text { Soil Depth is Unqualified }\end{array}$ \\
\hline $\begin{array}{l}\text { USW } \\
\text { UZ-N41 }\end{array}$ & 765867.75 & 563521.07 & MO9906GPS98410.000 [DIRS 109059] ${ }^{a}$ & 16.01 & 4.88 & $\begin{array}{l}\text { GS910808312212.001 } \\
\text { [DIRS 175972] }^{\dagger}\end{array}$ & $\begin{array}{l}\text { Location is Qualified and } \\
\text { Verified; } \\
\text { Soil Depth is Unqualified }\end{array}$ \\
\hline $\begin{array}{l}\text { USW } \\
\text { UZ-N42 }\end{array}$ & 765729.13 & 562858.62 & MO9906GPS98410.000 [DIRS 109059] & 0.00 & 0.00 & $\begin{array}{l}\text { GS910808312212.001 } \\
\text { [DIRS 175972] }^{\dagger}\end{array}$ & $\begin{array}{l}\text { Location is Qualified and } \\
\text { Verified; } \\
\text { Soil Depth is Unqualified }\end{array}$ \\
\hline $\begin{array}{l}\text { USW } \\
\text { UZ-N43 }\end{array}$ & 765997.47 & 563263.61 & MO9906GPS98410.000 [DIRS 109059] ${ }^{a}$ & 27.00 & 8.23 & $\begin{array}{l}\text { GS910808312212.001 } \\
\text { [DIRS 175972] }^{f}\end{array}$ & $\begin{array}{l}\text { Location is Qualified and } \\
\text { Verified; } \\
\text { Soil Depth is Unqualified }\end{array}$ \\
\hline $\begin{array}{l}\text { USW } \\
\text { UZ-N44 }\end{array}$ & 766193.00 & 563139.53 & MO9906GPS98410.000 [DIRS 109059] & 0.00 & 0.00 & $\begin{array}{l}\text { GS910808312212.001 } \\
\text { [DIRS 175972] }^{\dagger}\end{array}$ & $\begin{array}{l}\text { Location is Qualified and } \\
\text { Verified; } \\
\text { Soil Depth is Unqualified }\end{array}$ \\
\hline $\begin{array}{l}\text { USW } \\
\text { UZ-N45 }\end{array}$ & 765977.24 & 563429.26 & MO9906GPS98410.000 [DIRS 109059] ${ }^{a}$ & 35.01 & 10.67 & $\begin{array}{l}\text { GS910808312212.001 } \\
\text { [DIRS 175972] }^{\dagger}\end{array}$ & $\begin{array}{l}\text { Location is Qualified and } \\
\text { Verified; } \\
\text { Soil Depth is Unqualified }\end{array}$ \\
\hline
\end{tabular}


Table A-1. Boreholes and Surficial Deposit Depth Measurements (Continued)

\begin{tabular}{|c|c|c|c|c|c|c|c|}
\hline $\begin{array}{c}\text { Borehole } \\
\text { Name }\end{array}$ & $\begin{array}{c}\text { State } \\
\text { Plane } \\
\text { Coordinate } \\
\text { Northing }\end{array}$ & \begin{tabular}{|c} 
State \\
Plane \\
Coordinate \\
Easting
\end{tabular} & $\begin{array}{l}\text { Location Source by Author and Date, } \\
\text { or DTN, or Both }\end{array}$ & \begin{tabular}{|c|} 
Alluvial \\
$\begin{array}{c}\text { Thickness } \\
\text { (ft) }\end{array}$ \\
\end{tabular} & $\begin{array}{c}\text { Alluvial } \\
\text { Thickness } \\
\text { (m) }\end{array}$ & $\begin{array}{c}\text { Thickness Source by } \\
\text { Author and Date, or DTN, } \\
\text { or Both }\end{array}$ & Remarks \\
\hline \multicolumn{8}{|c|}{ UNQUALIFIED DATA (Continued) } \\
\hline $\begin{array}{l}\text { USW } \\
\text { UZ-N46 }\end{array}$ & 772262.19 & 559747.93 & MO9906GPS98410.000 [DIRS 109059] & 0.00 & 0.00 & $\begin{array}{l}\text { GS910808312212.001 } \\
\text { [DIRS 175972] }^{\dagger}\end{array}$ & $\begin{array}{l}\text { Location is Qualified and } \\
\text { Verified; } \\
\text { Soil Depth is Unqualified }\end{array}$ \\
\hline $\begin{array}{l}\text { USW } \\
\text { UZ-N47 }\end{array}$ & 771967.54 & 559783.74 & MO9906GPS98410.000 [DIRS 109059] & 33.01 & 10.06 & $\begin{array}{l}\text { GS910808312212.001 } \\
\text { [DIRS 175972] }^{\dagger}\end{array}$ & $\begin{array}{l}\text { Location is Qualified and } \\
\text { Verified; } \\
\text { Soil Depth is Unqualified }\end{array}$ \\
\hline $\begin{array}{l}\text { USW } \\
\text { UZ-N48 }\end{array}$ & 760835.94 & 562413.82 & MO9906GPS98410.000 [DIRS 109059] ${ }^{a}$ & 0.00 & 0.00 & $\begin{array}{l}\text { GS910808312212.001 } \\
\text { [DIRS 175972] }^{\dagger}\end{array}$ & $\begin{array}{l}\text { Location is Qualified and } \\
\text { Verified; } \\
\text { Soil Depth is Unqualified }\end{array}$ \\
\hline $\begin{array}{l}\text { USW } \\
\text { UZ-N49 }\end{array}$ & 760861.42 & 562322.06 & MO9906GPS98410.000 [DIRS 109059] & 2.00 & 0.61 & $\begin{array}{l}\text { GS910808312212.001 } \\
\text { [DIRS 175972] }^{\dagger}\end{array}$ & $\begin{array}{l}\text { Location is Qualified and } \\
\text { Verified; } \\
\text { Soil Depth is Unqualified }\end{array}$ \\
\hline $\begin{array}{l}\text { USW } \\
\text { UZ-N50 }\end{array}$ & 760776.92 & 562911.92 & MO9906GPS98410.000 [DIRS 109059] ${ }^{\mathrm{a}}$ & 8.99 & 2.74 & $\begin{array}{l}\text { GS910808312212.001 } \\
\text { [DIRS 175972] }^{\dagger}\end{array}$ & $\begin{array}{l}\text { Location is Qualified and } \\
\text { Verified; } \\
\text { Soil Depth is Unqualified }\end{array}$ \\
\hline $\begin{array}{l}\text { USW } \\
\text { UZ-N51 }\end{array}$ & 760861.75 & 562909.56 & MO9906GPS98410.000 [DIRS 109059] ${ }^{a}$ & 14.01 & 4.27 & $\begin{array}{l}\text { GS910808312212.001 } \\
\text { [DIRS 175972] }^{\dagger}\end{array}$ & $\begin{array}{l}\text { Location is Qualified and } \\
\text { Verified; } \\
\text { Soil Depth is Unqualified }\end{array}$ \\
\hline $\begin{array}{l}\text { USW } \\
\text { UZ-N52 }\end{array}$ & 760894.93 & 562908.95 & MO9906GPS98410.000 [DIRS 109059] ${ }^{a}$ & 6.99 & 2.13 & $\begin{array}{l}\text { GS910808312212.001 } \\
\text { [DIRS 175972] }^{\dagger}\end{array}$ & $\begin{array}{l}\text { Location is Qualified and } \\
\text { Verified; } \\
\text { Soil Depth is Unqualified }\end{array}$ \\
\hline $\begin{array}{l}\text { USW } \\
\text { UZ-N65 }\end{array}$ & 758627.34 & 562537.63 & MO9906GPS98410.000 [DIRS 109059] & 0.00 & 0.00 & $\begin{array}{l}\text { GS910808312212.001 } \\
\text { [DIRS 175972] }^{f}\end{array}$ & $\begin{array}{l}\text { Location is Qualified and } \\
\text { Verified; } \\
\text { Soil Depth is Unqualified }\end{array}$ \\
\hline $\begin{array}{l}\text { USW } \\
\text { UZ-N66 }\end{array}$ & 758433.89 & 561881.67 & MO9906GPS98410.000 [DIRS 109059] & 0.00 & 0.00 & $\begin{array}{l}\text { GS910808312212.001 } \\
\text { [DIRS 175972] }^{f}\end{array}$ & $\begin{array}{l}\text { Location is Qualified and } \\
\text { Verified; } \\
\text { Soil Depth is Unqualified }\end{array}$ \\
\hline $\begin{array}{l}\text { USW } \\
\text { UZ-N67 }\end{array}$ & 753635.58 & 563799.22 & MO9906GPS98410.000 [DIRS 109059] & 19.00 & 5.79 & $\begin{array}{l}\text { GS910808312212.001 } \\
\text { [DIRS 175972] }^{\dagger}\end{array}$ & $\begin{array}{l}\text { Location is Qualified and } \\
\text { Verified; } \\
\text { Soil Depth is Unqualified }\end{array}$ \\
\hline $\begin{array}{l}\text { USW } \\
\text { UZ-N68 }\end{array}$ & 753963.71 & 564006.12 & MO9906GPS98410.000 [DIRS 109059] ${ }^{a}$ & 50.00 & 15.24 & $\begin{array}{l}\text { GS910808312212.001 } \\
\text { [DIRS 175972] }^{f}\end{array}$ & $\begin{array}{l}\text { Location is Qualified and } \\
\text { Verified; } \\
\text { Soil Depth is Unqualified }\end{array}$ \\
\hline $\begin{array}{l}\text { USW } \\
\text { UZ-N69 }\end{array}$ & 754462.47 & 564401.98 & MO9906GPS98410.000 [DIRS 109059] & 27.00 & 8.23 & $\begin{array}{l}\text { GS910808312212.001 } \\
\text { [DIRS 175972] }^{f}\end{array}$ & $\begin{array}{l}\text { Location is Qualified and } \\
\text { Verified; } \\
\text { Soil Depth is Unqualified }\end{array}$ \\
\hline
\end{tabular}


Table A-1. Boreholes and Surficial Deposit Depth Measurements (Continued)

\begin{tabular}{|c|c|c|c|c|c|c|c|}
\hline $\begin{array}{c}\text { Borehole } \\
\text { Name }\end{array}$ & \begin{tabular}{|c|} 
State \\
Plane \\
Coordinate \\
Northing \\
\end{tabular} & \begin{tabular}{|c|} 
State \\
Plane \\
Coordinate \\
Easting
\end{tabular} & $\begin{array}{l}\text { Location Source by Author and Date, } \\
\text { or DTN, or Both }\end{array}$ & $\begin{array}{c}\text { Alluvial } \\
\text { Thickness } \\
\text { (ft) }\end{array}$ & $\begin{array}{c}\text { Alluvial } \\
\text { Thickness } \\
\text { (m) } \\
\end{array}$ & $\begin{array}{c}\text { Thickness Source by } \\
\text { Author and Date, or DTN, } \\
\text { or Both }\end{array}$ & Remarks \\
\hline \multicolumn{8}{|c|}{ UNQUALIFIED DATA (Continued) } \\
\hline $\begin{array}{l}\text { USW } \\
\text { UZ-N70 }\end{array}$ & 769250.78 & 560165.01 & MO9906GPS98410.000 [DIRS 109059] ${ }^{a}$ & 0.00 & 0.00 & $\begin{array}{l}\text { GS910808312212.001 } \\
\text { [DIRS 175972] }^{f}\end{array}$ & $\begin{array}{l}\text { Location is Qualified and } \\
\text { Verified; } \\
\text { Soil Depth is Unqualified }\end{array}$ \\
\hline $\begin{array}{l}\text { USW } \\
\text { UZ-N71 }\end{array}$ & 761025.67 & 558405.94 & MO9906GPS98410.000 [DIRS 109059] ${ }^{a}$ & 0.00 & 0.00 & $\begin{array}{l}\text { GS910808312212.001 } \\
\text { [DIRS 175972] }^{\dagger}\end{array}$ & $\begin{array}{l}\text { Location is Qualified and } \\
\text { Verified; } \\
\text { Soil Depth is Unqualified }\end{array}$ \\
\hline $\begin{array}{l}\text { USW } \\
\text { UZ-N72 }\end{array}$ & 761071.61 & 558645.91 & MO9906GPS98410.000 [DIRS 109059] ${ }^{\mathrm{a}}$ & 0.00 & 0.00 & $\begin{array}{l}\text { GS910808312212.001 } \\
\text { [DIRS 175972] }^{\dagger}\end{array}$ & $\begin{array}{l}\text { Location is Qualified and } \\
\text { Verified; } \\
\text { Soil Depth is Unqualified }\end{array}$ \\
\hline $\begin{array}{l}\text { USW } \\
\text { UZ-N73 }\end{array}$ & 761052.93 & 558945.57 & MO9906GPS98410.000 [DIRS 109059] & 0.00 & 0.00 & $\begin{array}{l}\text { GS910808312212.001 } \\
\text { [DIRS 175972] }^{\dagger}\end{array}$ & $\begin{array}{l}\text { Location is Qualified and } \\
\text { Verified; } \\
\text { Soil Depth is Unqualified }\end{array}$ \\
\hline $\begin{array}{l}\text { USW } \\
\text { UZ-N75 }\end{array}$ & 761462.24 & 559076.00 & MO9906GPS98410.000 [DIRS 109059] ${ }^{a}$ & 2.00 & 0.61 & $\begin{array}{l}\text { GS910808312212.001 } \\
\text { [DIRS 175972] }^{\dagger}\end{array}$ & $\begin{array}{l}\text { Location is Qualified and } \\
\text { Verified; } \\
\text { Soil Depth is Unqualified }\end{array}$ \\
\hline $\begin{array}{l}\text { USW } \\
\text { UZ-N76 }\end{array}$ & 761356.90 & 559067.41 & MO9906GPS98410.000 [DIRS 109059] ${ }^{a}$ & 0.00 & 0.00 & $\begin{array}{l}\text { GS910808312212.001 } \\
\text { [DIRS 175972] }^{\dagger}\end{array}$ & $\begin{array}{l}\text { Location is Qualified and } \\
\text { Verified; } \\
\text { Soil Depth is Unqualified }\end{array}$ \\
\hline $\begin{array}{l}\text { USW } \\
\text { UZ-N77 }\end{array}$ & 755526.90 & 554397.31 & MO9906GPS98410.000 [DIRS 109059] ${ }^{a}$ & 37.99 & 11.58 & $\begin{array}{l}\text { GS910808312212.001 } \\
\text { [DIRS 175972] }^{\dagger}\end{array}$ & $\begin{array}{l}\text { Location is Qualified and } \\
\text { Verified; } \\
\text { Soil Depth is Unqualified }\end{array}$ \\
\hline $\begin{array}{l}\text { USW } \\
\text { UZ-N78 }\end{array}$ & 757558.45 & 556262.68 & MO9906GPS98410.000 [DIRS 109059] ${ }^{a}$ & 0.00 & 0.00 & $\begin{array}{l}\text { GS910808312212.001 } \\
\text { [DIRS 175972] }^{\dagger}\end{array}$ & $\begin{array}{l}\text { Location is Qualified and } \\
\text { Verified; } \\
\text { Soil Depth is Unqualified }\end{array}$ \\
\hline $\begin{array}{l}\text { USW } \\
\text { UZ-N79 }\end{array}$ & 757733.70 & 556334.15 & MO9906GPS98410.000 [DIRS 109059] ${ }^{a}$ & 0.00 & 0.00 & $\begin{array}{l}\text { GS910808312212.001 } \\
\text { [DIRS 175972] }^{\dagger}\end{array}$ & $\begin{array}{l}\text { Location is Qualified and } \\
\text { Verified; } \\
\text { Soil Depth is Unqualified }\end{array}$ \\
\hline $\begin{array}{l}\text { USW } \\
\text { UZ-N81 }\end{array}$ & 757807.56 & 555595.39 & MO9906GPS98410.000 [DIRS 109059] & 6.00 & 1.83 & $\begin{array}{l}\text { GS910808312212.001 } \\
\text { [DIRS 175972] }^{\dagger}\end{array}$ & $\begin{array}{l}\text { Location is Qualified and } \\
\text { Verified; } \\
\text { Soil Depth is Unqualified }\end{array}$ \\
\hline
\end{tabular}


Table A-1. Boreholes and Surficial Deposit Depth Measurements (Continued)

\begin{tabular}{|c|c|c|c|c|c|c|c|}
\hline $\begin{array}{l}\text { Borehole } \\
\text { Name }\end{array}$ & \begin{tabular}{|c|} 
State \\
Plane \\
Coordinate \\
Northing \\
\end{tabular} & \begin{tabular}{|c} 
State \\
Plane \\
Coordinate \\
Easting
\end{tabular} & $\begin{array}{l}\text { Location Source by Author and Date, } \\
\text { or DTN, or Both }\end{array}$ & $\begin{array}{c}\text { Alluvial } \\
\begin{array}{c}\text { Thickness } \\
(\mathrm{ft})\end{array} \\
\end{array}$ & $\begin{array}{c}\text { Alluvial } \\
\text { Thickness } \\
\text { (m) }\end{array}$ & $\begin{array}{c}\text { Thickness Source by } \\
\text { Author and Date, or DTN, } \\
\text { or Both }\end{array}$ & Remarks \\
\hline \multicolumn{8}{|c|}{ UNQUALIFIED DATA (Continued) } \\
\hline $\begin{array}{l}\text { USW } \\
\text { UZ-N82 }\end{array}$ & 757498.76 & 554689.93 & MO9906GPS98410.000 [DIRS 109059] & 21.98 & 6.70 & $\begin{array}{l}\text { GS910808312212.001 } \\
\text { [DIRS 175972] }^{\dagger}\end{array}$ & $\begin{array}{l}\text { Location is Qualified and } \\
\text { Verified; } \\
\text { Soil Depth is Unqualified }\end{array}$ \\
\hline $\begin{array}{l}\text { USW } \\
\text { UZ-N83 }\end{array}$ & 760624.95 & 556349.23 & MO9906GPS98410.000 [DIRS 109059] & 0.00 & 0.00 & $\begin{array}{l}\text { GS910808312212.001 } \\
\text { [DIRS 175972] }^{\dagger}\end{array}$ & $\begin{array}{l}\text { Location is Qualified and } \\
\text { Verified; } \\
\text { Soil Depth is Unqualified }\end{array}$ \\
\hline $\begin{array}{l}\text { USW } \\
\text { UZ-N84 }\end{array}$ & 760717.05 & 555888.00 & MO9906GPS98410.000 [DIRS 109059] ${ }^{a}$ & 19.98 & 6.09 & $\begin{array}{l}\text { GS910808312212.001 } \\
\text { [DIRS 175972] }^{\dagger}\end{array}$ & $\begin{array}{l}\text { Location is Qualified and } \\
\text { Verified; } \\
\text { Soil Depth is Unqualified }\end{array}$ \\
\hline $\begin{array}{l}\text { USW } \\
\text { UZ-N86 }\end{array}$ & 760615.37 & 556460.50 & MO9906GPS98410.000 [DIRS 109059] ${ }^{a}$ & 0.00 & 0.00 & $\begin{array}{l}\text { GS910808312212.001 } \\
\text { [DIRS 175972] }^{\dagger}\end{array}$ & $\begin{array}{l}\text { Location is Qualified and } \\
\text { Verified; } \\
\text { Soil Depth is Unqualified }\end{array}$ \\
\hline $\begin{array}{l}\text { USW } \\
\text { UZ-N88 }\end{array}$ & 760797.96 & 556551.25 & MO9906GPS98410.000 [DIRS 109059] ${ }^{a}$ & 0.75 & 0.23 & $\begin{array}{l}\text { GS910808312212.001 } \\
\text { [DIRS 175972] }^{\dagger}\end{array}$ & $\begin{array}{l}\text { Location is Qualified and } \\
\text { Verified; } \\
\text { Soil Depth is Unqualified }\end{array}$ \\
\hline $\begin{array}{l}\text { USW } \\
\text { UZ-N89 }\end{array}$ & 760611.22 & 555588.72 & MO9906GPS98410.000 [DIRS 109059] ${ }^{a}$ & 33.01 & 10.06 & $\begin{array}{l}\text { GS910808312212.001 } \\
\text { [DIRS 175972] }^{\dagger}\end{array}$ & $\begin{array}{l}\text { Location is Qualified and } \\
\text { Verified; } \\
\text { Soil Depth is Unqualified }\end{array}$ \\
\hline $\begin{array}{l}\text { USW } \\
\text { UZ-N90 }\end{array}$ & 760609.25 & 555587.46 & MO9906GPS98410.000 [DIRS 109059] & 32.51 & 9.91 & $\begin{array}{l}\text { GS910808312212.001 } \\
\text { [DIRS 175972] }^{f}\end{array}$ & $\begin{array}{l}\text { Location is Qualified and } \\
\text { Verified; } \\
\text { Soil Depth is Unqualified }\end{array}$ \\
\hline $\begin{array}{l}\text { USW } \\
\text { UZ-N93 }\end{array}$ & 759584.09 & 558321.03 & MO9906GPS98410.000 [DIRS 109059] & 0.00 & 0.00 & $\begin{array}{l}\text { GS910808312212.001 } \\
\text { [DIRS 175972] }^{f}\end{array}$ & $\begin{array}{l}\text { Location is Qualified and } \\
\text { Verified; } \\
\text { Soil Depth is Unqualified }\end{array}$ \\
\hline $\begin{array}{l}\text { USW } \\
\text { UZ-N94 }\end{array}$ & 759723.29 & 558236.66 & MO9906GPS98410.000 [DIRS 109059] & 0.00 & 0.00 & $\begin{array}{l}\text { GS910808312212.001 } \\
\text { [DIRS 175972] }^{\dagger}\end{array}$ & $\begin{array}{l}\text { Location is Qualified and } \\
\text { Verified; } \\
\text { Soil Depth is Unqualified }\end{array}$ \\
\hline $\begin{array}{l}\text { USW } \\
\text { UZ-N96 }\end{array}$ & 759445.54 & 558403.36 & MO9906GPS98410.000 [DIRS 109059] & 2.00 & 0.61 & $\begin{array}{l}\text { GS910808312212.001 } \\
\text { [DIRS 175972] }^{f}\end{array}$ & $\begin{array}{l}\text { Location is Qualified and } \\
\text { Verified; } \\
\text { Soil Depth is Unqualified }\end{array}$ \\
\hline
\end{tabular}


Table A-1. Boreholes and Surficial Deposit Depth Measurements (Continued)

\begin{tabular}{|c|c|c|c|c|c|c|c|}
\hline $\begin{array}{c}\text { Borehole } \\
\text { Name }\end{array}$ & \begin{tabular}{|c|} 
State \\
Plane \\
Coordinate \\
Northing
\end{tabular} & \begin{tabular}{|c} 
State \\
Plane \\
Coordinate \\
Easting
\end{tabular} & $\begin{array}{l}\text { Location Source by Author and Date, } \\
\text { or DTN, or Both }\end{array}$ & \begin{tabular}{|c|} 
Alluvial \\
Thickness \\
(ft)
\end{tabular} & \begin{tabular}{|c} 
Alluvial \\
Thickness \\
(m)
\end{tabular} & $\begin{array}{c}\text { Thickness Source by } \\
\text { Author and Date, or DTN, } \\
\text { or Both }\end{array}$ & Remarks \\
\hline \multicolumn{8}{|c|}{ UNQUALIFIED DATA (Continued) } \\
\hline $\begin{array}{l}\text { USW } \\
\text { UZ-N98 }\end{array}$ & 767996.47 & 562083.75 & MO9906GPS98410.000 [DIRS 109059] & 0.98 & 0.30 & $\begin{array}{l}\text { GS910808312212.001 } \\
\text { [DIRS 175972] }^{f}\end{array}$ & $\begin{array}{l}\text { Location is Qualified and } \\
\text { Verified; } \\
\text { Soil Depth is Unqualified }\end{array}$ \\
\hline $\begin{array}{l}\text { USW } \\
\text { UZ-N24 }\end{array}$ & 768005.60 & 562054.42 & MO9906GPS98410.000 [DIRS 109059] ${ }^{a}$ & 0.49 & 0.15 & $\begin{array}{l}\text { GS910808312212.001 } \\
\text { [DIRS 175972] }^{\dagger}\end{array}$ & $\begin{array}{l}\text { Location is Qualified and } \\
\text { Verified; } \\
\text { Soil Depth is Unqualified }\end{array}$ \\
\hline $\begin{array}{l}\text { USW } \\
\text { UZ-N25 }\end{array}$ & 768430.44 & 561219.10 & MO9906GPS98410.000 [DIRS 109059] ${ }^{a}$ & 0.00 & 0.00 & $\begin{array}{l}\text { GS910808312212.001 } \\
\text { [DIRS 175972] }^{\dagger}\end{array}$ & $\begin{array}{l}\text { Location is Qualified and } \\
\text { Verified; Soil Depth is } \\
\text { Unqualified }\end{array}$ \\
\hline $\begin{array}{l}\text { USW } \\
\text { UZ-N26 }\end{array}$ & 768757.35 & 561023.24 & MO9906GPS98410.000 [DIRS 109059] & 0.00 & 0.00 & $\begin{array}{l}\text { GS910808312212.001 } \\
\text { [DIRS 175972] }^{f}\end{array}$ & $\begin{array}{l}\text { Location is Qualified and } \\
\text { Verified; } \\
\text { Soil Depth is Unqualified }\end{array}$ \\
\hline $\begin{array}{l}\text { UE-25 } \\
\text { UZN\#1 }\end{array}$ & 769329.75 & 565224.45 & MO9906GPS98410.000 [DIRS 109059] & 27.30 & 8.32 & $\begin{array}{l}\text { GS910808312212.001 } \\
\text { [DIRS 175972] }^{f}\end{array}$ & $\begin{array}{l}\text { Location is Qualified and } \\
\text { Verified; } \\
\text { Soil Depth is Unqualified }\end{array}$ \\
\hline $\begin{array}{l}\text { UE-25 } \\
\text { UZN\#2 }\end{array}$ & 768606.38 & 566113.70 & MO9906GPS98410.000 [DIRS 109059] ${ }^{a}$ & 0.00 & 0.00 & $\begin{array}{l}\text { GS910808312212.001 } \\
\text { [DIRS 175972] }^{\dagger}\end{array}$ & $\begin{array}{l}\text { Location is Qualified and } \\
\text { Verified; } \\
\text { Soil Depth is Unqualified }\end{array}$ \\
\hline $\begin{array}{l}\text { UE-25 } \\
\text { UZN\#3 }\end{array}$ & 768631.42 & 566119.42 & MO9906GPS98410.000 [DIRS 109059] ${ }^{a}$ & 8.99 & 2.74 & $\begin{array}{l}\text { GS910808312212.001 } \\
\text { [DIRS 175972] }^{\dagger}\end{array}$ & $\begin{array}{l}\text { Location is Qualified and } \\
\text { Verified; } \\
\text { Soil Depth is Unqualified }\end{array}$ \\
\hline $\begin{array}{l}\text { UE-25 } \\
\text { UZN\#4 }\end{array}$ & 768664.37 & 566127.14 & MO9906GPS98410.000 [DIRS 109059] & 24.51 & 7.47 & $\begin{array}{l}\text { GS910808312212.001 } \\
\text { [DIRS 175972] }^{f}\end{array}$ & $\begin{array}{l}\text { Location is Qualified and } \\
\text { Verified; } \\
\text { Soil Depth is Unqualified }\end{array}$ \\
\hline $\begin{array}{l}\text { UE-25 } \\
\text { UZN\#5 }\end{array}$ & 768690.17 & 566134.21 & MO9906GPS98410.000 [DIRS 109059] ${ }^{a}$ & 44.49 & 13.56 & $\begin{array}{l}\text { GS910808312212.001 } \\
\text { [DIRS 175972] }^{f}\end{array}$ & $\begin{array}{l}\text { Location is Qualified and } \\
\text { Verified; } \\
\text { Soil Depth is Unqualified }\end{array}$ \\
\hline $\begin{array}{l}\text { UE-25 } \\
\text { UZN\#6 }\end{array}$ & 768706.49 & 566136.79 & MO9906GPS98410.000 [DIRS 109059] & 39.50 & 12.04 & $\begin{array}{l}\text { GS910808312212.001 } \\
\text { [DIRS 175972] }^{f}\end{array}$ & $\begin{array}{l}\text { Location is Qualified and } \\
\text { Verified; } \\
\text { Soil Depth is Unqualified }\end{array}$ \\
\hline $\begin{array}{l}\text { UE-25 } \\
\text { UZN\#7 }\end{array}$ & 768724.95 & 566141.35 & MO9906GPS98410.000 [DIRS 109059] & 40.49 & 12.34 & $\begin{array}{l}\text { GS910808312212.001 } \\
\text { [DIRS 175972] }^{f}\end{array}$ & $\begin{array}{l}\text { Location is Qualified and } \\
\text { Verified; } \\
\text { Soil Depth is Unqualified }\end{array}$ \\
\hline $\begin{array}{l}\text { UE-25 } \\
\text { UZN\#8 }\end{array}$ & 768743.84 & 566146.64 & MO9906GPS98410.000 [DIRS 109059] & 39.99 & 12.19 & $\begin{array}{l}\text { GS910808312212.001 } \\
\text { [DIRS 175972] }^{\dagger}\end{array}$ & $\begin{array}{l}\text { Location is Qualified and } \\
\text { Verified; } \\
\text { Soil Depth is Unqualified }\end{array}$ \\
\hline
\end{tabular}


Table A-1. Boreholes and Surficial Deposit Depth Measurements (Continued)

\begin{tabular}{|c|c|c|c|c|c|c|c|}
\hline $\begin{array}{c}\text { Borehole } \\
\text { Name }\end{array}$ & $\begin{array}{c}\text { State } \\
\text { Plane } \\
\text { Coordinate } \\
\text { Northing } \\
\end{array}$ & \begin{tabular}{|c} 
State \\
Plane \\
Coordinate \\
Easting
\end{tabular} & $\begin{array}{c}\text { Location Source by Author and Date, } \\
\text { or DTN, or Both }\end{array}$ & $\begin{array}{c}\text { Alluvial } \\
\begin{array}{c}\text { Thickness } \\
(\mathrm{ft})\end{array} \\
\end{array}$ & $\begin{array}{c}\text { Alluvial } \\
\text { Thickness } \\
\text { (m) }\end{array}$ & $\begin{array}{c}\text { Thickness Source by } \\
\text { Author and Date, or DTN, } \\
\text { or Both }\end{array}$ & Remarks \\
\hline \multicolumn{8}{|c|}{ UNQUALIFIED DATA (Continued) } \\
\hline $\begin{array}{l}\text { UE-25 } \\
\text { UZN\#9 }\end{array}$ & 768782.36 & 566156.06 & MO9906GPS98410.000 [DIRS 109059] & 35.01 & 10.67 & $\begin{array}{l}\text { GS910808312212.001 } \\
\text { [DIRS 175972] }^{\dagger}\end{array}$ & $\begin{array}{l}\text { Location is Qualified and } \\
\text { Verified; } \\
\text { Soil Depth is Unqualified }\end{array}$ \\
\hline $\begin{array}{l}\text { UE-25 } \\
\text { UZN\#10 }\end{array}$ & 769869.29 & 564744.59 & MO9906GPS98410.000 [DIRS 109059] & 0.00 & 0.00 & $\begin{array}{l}\text { GS910808312212.001 } \\
\text { [DIRS 175972] }^{\dagger}\end{array}$ & $\begin{array}{l}\text { Location is Qualified and } \\
\text { Verified; } \\
\text { Soil Depth is Unqualified }\end{array}$ \\
\hline $\begin{array}{l}\text { UE-25 } \\
\text { UZN\#12 }\end{array}$ & 768651.53 & 566695.21 & MO9906GPS98410.000 [DIRS 109059] ${ }^{a}$ & 45.01 & 13.72 & $\begin{array}{l}\text { GS910808312212.001 } \\
\text { [DIRS 175972] }^{\dagger}\end{array}$ & $\begin{array}{l}\text { Location is Qualified and } \\
\text { Verified; } \\
\text { Soil Depth is Unqualified }\end{array}$ \\
\hline $\begin{array}{l}\text { UE-25 } \\
\text { UZN\#13 }\end{array}$ & 768025.46 & 568255.20 & MO9906GPS98410.000 [DIRS 109059] & 44.00 & 13.41 & $\begin{array}{l}\text { GS910808312212.001 } \\
\text { [DIRS 175972] }^{\dagger}\end{array}$ & $\begin{array}{l}\text { Location is Qualified and } \\
\text { Verified; } \\
\text { Soil Depth is Unqualified }\end{array}$ \\
\hline $\begin{array}{l}\text { UE-25 } \\
\text { UZN\#14 }\end{array}$ & 767968.00 & 568233.04 & MO9906GPS98410.000 [DIRS 109059] ${ }^{\mathrm{a}}$ & 44.00 & 13.41 & $\begin{array}{l}\text { GS910808312212.001 } \\
\text { [DIRS 175972] }^{\dagger}\end{array}$ & $\begin{array}{l}\text { Location is Qualified and } \\
\text { Verified; } \\
\text { Soil Depth is Unqualified }\end{array}$ \\
\hline $\begin{array}{l}\text { UE-25 } \\
\text { UZN\#18 }\end{array}$ & 766472.79 & 565246.63 & MO9906GPS98410.000 [DIRS 109059] ${ }^{a}$ & 56.00 & 17.07 & $\begin{array}{l}\text { GS910808312212.001 } \\
\text { [DIRS 175972] }^{\dagger}\end{array}$ & $\begin{array}{l}\text { Location is Qualified and } \\
\text { Verified; } \\
\text { Soil Depth is Unqualified }\end{array}$ \\
\hline $\begin{array}{l}\text { UE-25 } \\
\text { UZN\#19 }\end{array}$ & 763689.57 & 564570.82 & MO9906GPS98410.000 [DIRS 109059] ${ }^{a}$ & 22.51 & 6.86 & $\begin{array}{l}\text { GS910808312212.001 } \\
\text { [DIRS 175972] }^{\dagger}\end{array}$ & $\begin{array}{l}\text { Location is Qualified and } \\
\text { Verified; } \\
\text { Soil Depth is Unqualified }\end{array}$ \\
\hline $\begin{array}{l}\text { UE-25 } \\
\text { UZN\#20 }\end{array}$ & 763760.60 & 564579.42 & MO9906GPS98410.000 [DIRS 109059] & 39.01 & 11.89 & $\begin{array}{l}\text { GS910808312212.001 } \\
\text { [DIRS 175972] }^{f}\end{array}$ & $\begin{array}{l}\text { Location is Qualified and } \\
\text { Verified; } \\
\text { Soil Depth is Unqualified }\end{array}$ \\
\hline $\begin{array}{l}\text { UE-25 } \\
\text { UZN\#21 }\end{array}$ & 763806.82 & 564591.36 & MO9906GPS98410.000 [DIRS 109059] & 27.49 & 8.38 & $\begin{array}{l}\text { GS910808312212.001 } \\
\text { [DIRS 175972] }^{f}\end{array}$ & $\begin{array}{l}\text { Location is Qualified and } \\
\text { Verified; } \\
\text { Soil Depth is Unqualified }\end{array}$ \\
\hline $\begin{array}{l}\text { UE-25 } \\
\text { UZN\#22 }\end{array}$ & 763881.05 & 564604.88 & MO9906GPS98410.000 [DIRS 109059] & 19.49 & 5.94 & $\begin{array}{l}\text { GS910808312212.001 } \\
\text { [DIRS 175972] }^{f}\end{array}$ & $\begin{array}{l}\text { Location is Qualified and } \\
\text { Verified; } \\
\text { Soil Depth is Unqualified }\end{array}$ \\
\hline $\begin{array}{l}\text { UE-25 } \\
\text { UZN\#23 }\end{array}$ & 763973.76 & 564545.69 & MO9906GPS98410.000 [DIRS 109059] ${ }^{a}$ & 0.00 & 0.00 & $\begin{array}{l}\text { GS910808312212.001 } \\
\text { [DIRS 175972] }^{f}\end{array}$ & $\begin{array}{l}\text { Location is Qualified and } \\
\text { Verified; } \\
\text { Soil Depth is Unqualified }\end{array}$ \\
\hline $\begin{array}{l}\text { UE-25 } \\
\text { UZN\#29 }\end{array}$ & 762613.84 & 565173.32 & MO9906GPS98410.000 [DIRS 109059] & 0.98 & 0.30 & $\begin{array}{l}\text { GS910808312212.001 } \\
\text { [DIRS 175972] }^{f}\end{array}$ & $\begin{array}{l}\text { Location is Qualified and } \\
\text { Verified; } \\
\text { Soil Depth is Unqualified }\end{array}$ \\
\hline
\end{tabular}


Table A-1. Boreholes and Surficial Deposit Depth Measurements (Continued)

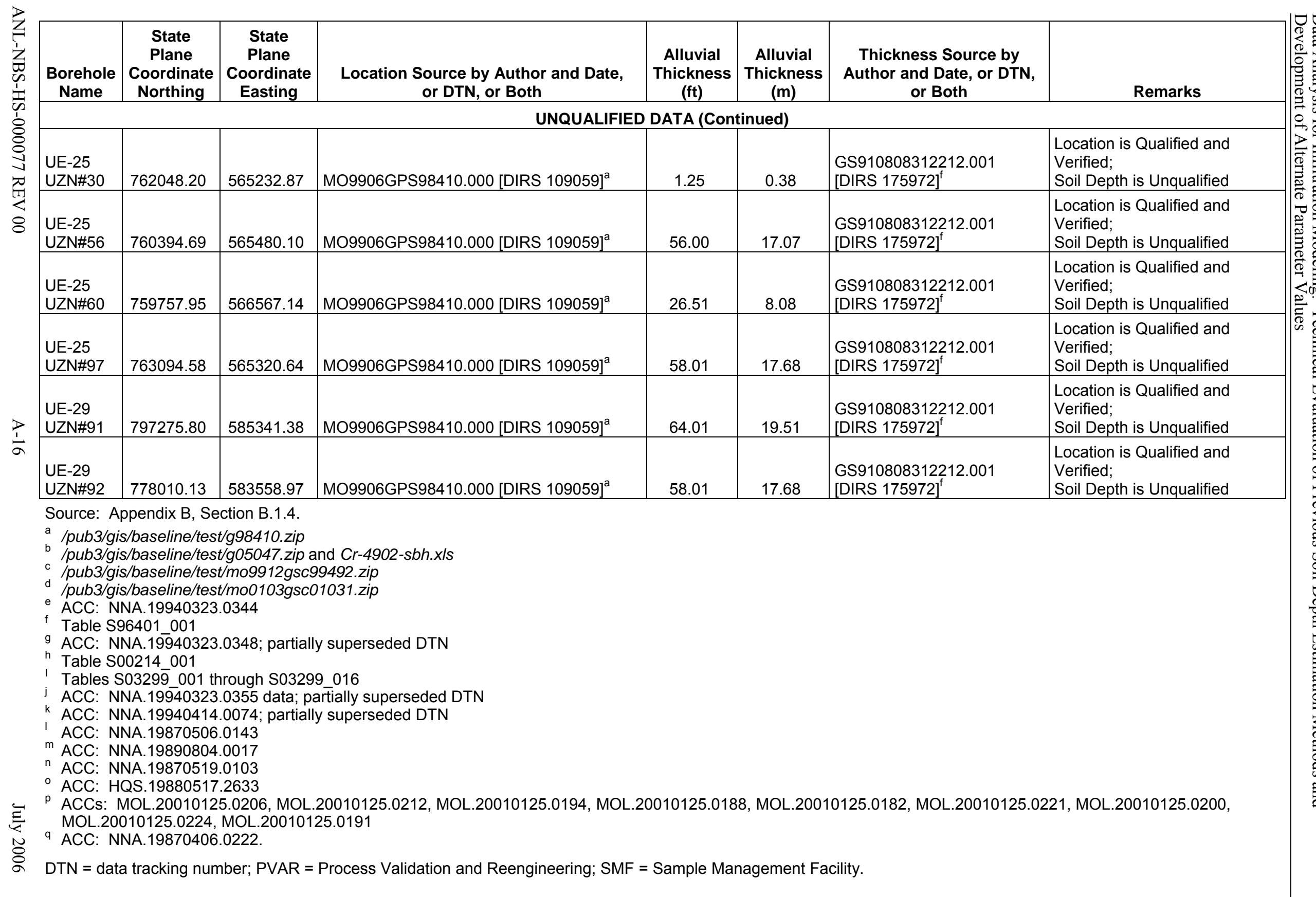


APPENDIX B

ELECTRONIC SPREADSHEETS AND WORKBOOK-WORKSHEETS FOR CALCULATIONS PRESENTED IN THE ANALYSIS 


\section{B1. SUPPORTING INFORMATION FOR ELECTRONIC SPREADSHEETS AND WORKBOOK-WORKSHEETS FOR CALCULATIONS PRESENTED IN THE ANALYSIS}

Included with this appendix is a compact disc with copies of Excel® spreadsheets and workbooks with worksheets used to create the tables, charts, and values presented in this analysis in their native format. The provision of this information responds to the requirements of LP-SIII.9Q-BSC, Attachment 2, regarding the use of standard functions in exempted software, Excel ${ }^{\circledR}$, used for this analysis. Several spreadsheets and worksheets were used to carry out operations in Excel ${ }^{\circledR}$. The following text provides a roadmap and explanation to allow the user to locate the proper spreadsheet or worksheet, and to interpret the material contained in the spreadsheet or worksheet for a particular table, figure, or text. The user can determine the standard function used to calculate a particular value in a cell on a spreadsheet or worksheet by selecting the cell and finding the function on the formula bar. The software ARCINFO was also used to support the comparison of historic and revised map files.

\section{B.1.1 Files for Section 6.1.2 - Comparison of SOILMAP6.INP to the Digital Soil Map}

A comparison was made (DTN: MO0512SPASURFD.000 [DIRS 175870]) between the version of the soil map in SOILMAP6.INP (USGS 2000 [DIRS 175858], folder Soilmap6) and the digital version of the original source map (DTN: MO9903COV96274.000 [DIRS 176571], /publ3/gis/baseline/geol/soils.e00). The comparison was performed using the ARCINFO IDENTITY command. The summary statistics for that comparison are given in Guertal Test.xls.

\section{B.1.2 Files for Section 6.1.2 - Check of Soil Depth Code Equation Calculation}

A check was performed of how well the equations, given to calculate depth from topographic slope, were implemented in SOILMAP6.INP (USGS 2000 [DIRS 175858], folder Soilmap6). Depth eq. test.xls documents the check performed using a single spreadsheet or worksheet containing the Excel ${ }^{\circledR}$ maximum of 65,536 rows. Columns A through $\mathrm{K}$ were copied from the first 65,536 rows in SOILMAP6.INP and pasted into Depth eq. test.xls. The spreadsheet was then sorted on slope angle (column F) and soil depth code (column J) to produce the version of the file displayed in the spreadsheet. The calculated soil depth was then calculated independently in column L using the equations shown in Section 6.1.2. Column M represents the difference between the file value (column K) and the independently calculated value (column L). Column $\mathrm{M}$ values were then summed to test for any non-zero differences (cell Q12). The result identifies some differences, those being a series of 0.01 values that result from rounding to two decimal places in the calculation. Row 11,232 provides an example of the rounding issue.

\section{B.1.3 Files for Table 6-3, Figures 6-4 and 6-5, and Associated Text in Section 6.1.2}

Table 6-3 and Figures 6-4 and 6-5 compare the soil depth equations to a set of corroborating data (DTN: GS011208312212.004 [DIRS 176317], Table S02086_001) that provide field measurements of the topographic slope and associated soil depth. These items were compiled in Taylor soil depth.xls using several worksheets. The original data (DTN: GS011208312212.004 [DIRS 176317], Table S02086_001) are shown in worksheet 'Original Data'. Taylor soil 
depth.xls also includes location information from the scientific notebook associated with DTN: GS011208312212.004 [DIRS 176317], Table S02086_001. The values shown in Table 6-3 and on Figures 6-4 and 6-5, and in text, were derived in worksheet 'Final calc'. Values representing Soil Depth Code 1 were selected and copied from worksheet 'Original Data' and appear in columns A through I. These values were sorted on the slope magnitude value (column F). Values calculated from the field slope measurements using the equations from Section 6.1.2 are given in column J. The difference between the calculated and measured soil depths is given in column $\mathrm{K}$. The correlation coefficient between the predicted depth from the equations and the measured depths is given in cells Q31 and Q65 for the two ranges of slope angles. The correlation coefficient for the range between 10 and 40 degrees with outliers removed is calculated in cell AD80.

\section{B.1.4 Files for Table A-1}

Information presented in Table A-1 were compiled in worksheet 'BH compile' located in Final calcs. for report.xls. Sources that were reviewed in compiling data for Table A-1 are shown in 'BH compile', while Table A-1 lists values and the sources of those values. Thus, some additional records may appear in 'BH compile' as sources. Data were copied from 'BH compile' for use in other worksheets in preparing other tables.

\section{B.1.5 Files for Table 6-4 and Figure 6-10}

Information presented in Table 6-4 were compiled in Final calcs. for report.xls using several worksheets. Borehole location data (DTN: MO0512SPASURFD.000 [DIRS 175870]) were taken from worksheet 'BH compile' and from SOILMAP6.INP (USGS 2000 [DIRS 175858], folder Soilmap6), then ARCINFO was used to determine the calculated soil thickness and soil depth code for the grid cell in which each borehole is located. The results are shown on worksheet 'Code assign' in columns E and F. Relevant data were then copied to worksheet 'Soilmap BH diff' as columns A through D and sorted by the SOILMAP6.INP soil depth code. The difference between the measured borehole soil depth and the SOILMAP6.INP calculated depth was then calculated in column F of 'Soilmap BH diff'. The column F values are shown as an absolute difference in column E. Averages for the absolute differences are calculated for the depth codes in column H. Differences were not calculated for Soil Depth Code 4, where the borehole depth exceeded $6 \mathrm{~m}$, because the result would not be meaningful; soil depth code uses a constant value of $6 \mathrm{~m}$ and, as long as the borehole value exceeded the constant, the difference between the two measures provides no information on the accuracy of the SOILMAP6.INP prediction. Relevant columns were copied to columns M through O of 'Soilmap BH diff' to create Figure 6-10.

\section{B.1.6 Files for Tables 6-7, 6-8, and 6-9, and Figures 6-15, 6-17, and 6-19}

Information presented in Tables 6-7 to 6-9, and in Figures 6-15, 6-17, and 6-19, were compiled in Final calcs. for report.xls using several worksheets. Borehole location data were taken from worksheet 'BH compile' and from output DTN: MO0606SPASDFIM.005 for soil depth classes (Figure 6-12), then ARCINFO was used to determine the soil depth class for the grid cell in which each borehole is located. The results are shown on worksheet 'Code assign' in column I and on worksheet 'Borehole depth class' in column J. The data in 'Borehole depth class' were 
then transferred to worksheet 'Sorted Boreholes' and sorted by soil depth class. The appropriate segment of data for each depth class was then copied and transferred to worksheets 'Class 3', 'Class 2', and 'Class 1' in columns A through F. In 'Class 3' the modified depths, natural logarithm values, mean, and standard deviation appear in columns $\mathrm{S}$ and $\mathrm{Z}$ through $\mathrm{AA}$, respectively. The sample median, which is also the estimated population median (Gilbert 1987 [DIRS 163705], p. 171), is calculated in cell AC2 using the standard Excel ${ }^{\circledR}$ function. For the mean and the standard deviation calculations, five additional values were added from the field measurements, which are shown in cells S12 through S16 of 'Class 3' in Final calcs. for report.xls; these values are from worksheets 'Original Data' and 'Class 3' of Taylor soil depth.xls. The calculation for the histograms on Figure 6-15 is shown in 'Class 3' of Final calcs. for report.xls, in columns I through $\mathrm{P}$ for the linear version of the histogram and in columns AE through AI for the log transformed version of the histogram.

Worksheet 'Class 2' of Final calcs. for report.xls does not use a lognormal distribution and, therefore, does not display columns related to this calculation. Two different calculations of the sample statistics are presented, one set with the lower bound truncation value at $0.00 \mathrm{~m}$ and another with the truncation value at $0.5 \mathrm{~m}$. The sample mean, sample standard deviation, and sample median, are calculated in cells S2, T2, and U2 for a lower bound of $0.0 \mathrm{~m}$ and in cells R14, S14, and T14 for a lower bound of $0.5 \mathrm{~m}$.. The sample median, which is also the estimated population median (Gilbert 1987 [DIRS 163705], p. 171), is calculated in cell U2 using the standard Excel ${ }^{\circledR}$ function. The calculation for the histogram appears in columns $J$ through $P$.

Worksheet 'Class 1' of Final calcs. for report.xls shows the only calculation for the histogram, being that a uniform distribution was used and no other calculations were necessary.

\section{B.1.7 Files for Figure 6-11}

Information presented in Figure 6-11 were compiled in Final calcs. for report.xls. Borehole location data were taken from worksheet 'BH compile' and from output DTN: MO0606SPASDFIM.005 for soil depth classes (Figure 6-12), then ARCINFO was used to determine the soil depth class for the grid cell in which each borehole is located. The results are shown on worksheet 'Code assign' in column I and on worksheet 'Borehole depth class' in column J. The data in 'Borehole depth class' were transferred to worksheet 'Sorted Boreholes' and were subsequently sorted by soil depth class. The borehole data from Midway Valley were then copied to worksheet 'Midway Valley depth'. The distance east of the UE-25 NRG\#1 borehole was calculated by subtracting the State Plane easting coordinate for UE-25 NRG\#1 from the easting coordinates for the boreholes on the list in column G. This result was converted from feet to meters in column $\mathrm{H}$.

\section{B.1.8 Files for Section 6.2.1 - W Test for DTN: GS011208312212.004, Calculation for Mean and Standard Deviation for Class 4, Table 6-6, and Figures 6-13 and 6-14}

Section 6.2.1 discusses a W test for a field measurement data set that provides soil depth (DTN: GS011208312212.004 [DIRS 176317], Table S02086_001). Data used to conduct the test are from Taylor soil depth.xls. The original data, copied from DTN: GS011208312212.004 [DIRS 176317], Table S02086_001, are shown on worksheet 'Original Data'. This worksheet 
includes location information from the scientific notebook associated with the DTN. The depths interpreted as belonging to Soil Class 4 were copied to column A of worksheet 'Wtest' and then sorted. The natural logarithm value for the depths was calculated in column B. The actual $\mathrm{W}$ test for the lognormal hypothesis is carried out in columns $\mathrm{W}$ through $\mathrm{Z}$. The equation for calculating the "W" value was implemented as a series of steps using basic Excel ${ }^{\circledR}$ functions in cells Y38 through Y45. A parallel W test for the normal hypothesis is shown in columns AC through AE. The lognormal mean and standard deviation for this data set are also calculated for the distribution for Soil Depth Class 4 (Section 6.2.1). These values are calculated in cells G21 and G22. The sample median, which is also the estimated population median (Gilbert 1987 [DIRS 163705], p. 171), is calculated in cell J21 using the standard Excel ${ }^{\circledR}$ function.

The data shown on Table 6-6 are from the listing in columns A and B of worksheet 'Wtest'. This listing was extracted from the original data copied from DTN: GS011208312212.004 [DIRS 176317], Table S02086_001 in worksheet 'Original Data'. Measurements with a "Deposit Unit" designation of "Tpc" or "stone st" were interpreted as belonging to Soil Depth Class 4 (Section 6.2.1). Figure 6-13 was generated on 'Wtest' from the data in columns A and B and from the histogram data in cells F3 to F18, G3 to G18, I3 to I15, and J3 to J15, which were generated using the HISTOGRAM function from the Excel ${ }^{\circledR}$ Data Analysis menu. Figure 6-14 was generated from the calculations shown in columns AI through AN on 'Wtest'.

\section{B.1.9 Files for Section 6.2.1 - W Test for Boreholes in Class 4 and Figure 6-15}

The W test for the Soil Depth Class 4 borehole data is documented in Final calcs. for report.xls. The modified depth values related to Soil Depth Class 4 were extracted from worksheet 'Class 4' (Section B.1.6), pasted into column A of worksheet 'W-test C4', and then sorted. The natural logarithm value for the depth was calculated in column B and the square of the natural logarithm value was calculated in column $\mathrm{C}$. The equation for calculating the $\mathrm{W}$ value is implemented as a series of steps using basic Excel ${ }^{\circledR}$ functions in cells B25 through B35. A parallel W test for the normal hypothesis is shown in columns D through F. A second parallel W test for the normal hypothesis using unmodified data is shown in columns $\mathrm{H}$ through $\mathrm{J}$. The values for the probability plot shown in Figure 6-15 are calculated in columns N through S.

\section{B.1.10 Files for Section 6.2.1 - W Test for Class 3 and Figure 6-16}

The W test for the Soil Depth Class 3 data is documented in Final calcs. for report.xls. The modified depth values for the boreholes related to Soil Depth Class 3 and the field measurements were extracted from worksheet 'Class 3' (Section B.1.6), pasted into column S of worksheet 'W-test C3', and then sorted. The natural logarithm value for the depth was calculated in column $\mathrm{T}$ and the square of the natural logarithm value was calculated in column $\mathrm{U}$. The equation for calculating the $\mathrm{W}$ value is implemented as a series of steps using basic Excel ${ }^{\circledR}$ functions in cells T20 to T33. A parallel W test for the normal hypothesis is shown in columns $\mathrm{W}$ to $\mathrm{X}$. The probability plot illustrated in Figure 6-16 is calculated in columns AB through AG.

\section{B.1.11 Files for Section 6.2.1 - D’Agostino's Test for Class 2 and Figure 6-18}

D'Agostino's Test for the Soil Depth Class 2 data is documented in Final calcs. for report.xls. The modified depth values related to Soil Depth Class 2 were extracted from worksheet 'Class 2' 
(Section B.1.6), pasted into column A of worksheet 'DAg test', and then sorted. Parameters related to the test are calculated in columns B through $\mathrm{F}$. The equation for calculating the Y value of the test is implemented as a series of steps using basic Excel ${ }^{\circledR}$ functions in cells D68 to D70, F71 to F73, and B74 to B75. The values for significance levels used to compare with the $\mathrm{Y}$ value are given in cells B76 to B77 and $\mathrm{C} 76$ to $\mathrm{C} 77$. The calculation of the values shown on the probability plot shown in Figure 6-18 is given in columns K, L, M, P, and Q. Columns N, O, $\mathrm{R}$, and $\mathrm{S}$ relate to a probability plot (not shown) to test the effect of the truncation at zero on the test results. Column $\mathrm{T}$ relates to a probability plot (not shown) for the normal logarithm hypothesis.

\section{B.1.12 Files for Section 6.2.2 and Table 6-10 - Confidence Intervals}

The confidence intervals for the Soil Depth Class 4 mean are calculated on worksheet 'Wtest' in Taylor soil depth.xls. The sample mean and the sample standard deviation are given in cells G21 and G22. The estimated population mean, calculated as the sample mean plus one-half the sample variance, is calculated in cells G25 and G26. Two methods were considered for calculating the estimated population mean (Gilbert 1987 [DIRS 163705]). This analysis uses the "Less Efficient But Simpler Estimator" (Gilbert 1987 [DIRS 163705], Equation 13.7) rather than an alternative method (Gilbert 1987 [DIRS 163705], Equation 13.13), because the approach does not require interpolating a $\psi$ value from a table and the equations for the confidence limits have the estimated population mean from Equation 13.7 embedded in them.

A comparison of the results between the two approaches is shown in cells F56, E56, and G26 on worksheet 'Wtest' in Taylor soil depth.xls. The comparison indicates that the difference between the two approaches is less than $1 \%$. A second comparison between the two equations for the Soil Depth Class 3 estimated population mean is shown on worksheet 'Class 3' of Final calcs. for report.xls in cells W29 to W31 and W5. This comparison shows a somewhat larger difference of 5.6\%. The upper and lower confidence limits for Soil Class 4 are calculated using equations and tables (Gilbert 1987 [DIRS 163705], Equations 13.13 and 13.14; Tables A10 to A13) in cells G33 through G49 on 'Wtest'. The " $H$ " values used in the calculation were taken from the tables using the $\mathrm{n}$ and $\mathrm{s}_{\mathrm{y}}$ values closest to the sample value, meaning that no interpolation was performed. Interpolation was not performed because the differences between adjacent values on the source tables are small and do not significantly affect the calculation.

The confidence intervals for the Soil Depth Class 3 mean are calculated in similar fashion on worksheet 'Class 3' in Final calcs. for report.xls. The sample mean and the sample standard deviation are given in cells Z2 and AA2. The estimated population mean is calculated in cells W4 and W5. The upper and lower confidence limits are calculated in cells W10 through W24. The confidence intervals for the Soil Depth Class 2 mean are calculated using the Student's t-distribution on worksheet 'Class 2' in Final calcs. for report.xls. The sample mean and the sample standard deviation are given in cells R2 and S2. The upper and lower confidence limits are calculated in cells R5 to R12, S10 to S12, and T10 to T12. The estimated population mean for Soil Depth Class 1 is calculated as $0.5(40+150)$. Confidence limits are not provided for this class because they are not meaningful for this case. The confidence intervals for the Soil Depth Class 4 median are calculated on worksheet 'Wtest' in Taylor soil depth.xls using equation 13.20 by Gilbert (1987 [DIRS 163705]). The equation is implemented as a series of Excel ${ }^{\circledR}$ calculations in cells G53 to G66, H53 to H66, and I53 to I64. The confidence intervals for the 
Soil Depth Class 3 median are calculated in similar fashion on worksheet 'Class 3' in Final calcs. for report.xls. The equation is implemented as a series of Excel ${ }^{\circledR}$ calculations in cells $\mathrm{AA} 3$ to $\mathrm{AA} 15, \mathrm{AB} 3$ to $\mathrm{AB} 15$, and $\mathrm{AC} 3$ to $\mathrm{AC} 13$.

\section{B.1.13 Files for Figure 7-1}

Figure 7-1 was generated on worksheet 'Figure 7-1' in Section 7 comparison.xls. Values appearing in Depth eq. test.xls (Section B.1.2) for Soil Depth Code 1 were copied and pasted into Section 7 comparison.xls. These data were then plotted using the HISTOGRAM function from the Excel ${ }^{\circledR}$ Data Analysis menu in cells D52 through D67 and E52 through E67. The mean for these data are calculated in cell E22. Values for the sample mean and the sample standard deviation for Soil Depth Class 4 (Table 6-10) were then used to create a hypothetical distribution for the same number of values. This distribution is calculated in cells D32 though I44.

\section{B2. COMPACT DISC FOR ELECTRONIC SPREADSHEETS AND WORKBOOK- WORKSHEETS FOR CALCULATIONS PRESENTED IN THE ANALYSIS}

The compact disc that accompanies this analysis contains the following files as part of Appendix B:

- Depth eq. test.xls

- Final calcs. For report.xls

- Guertal Test.xls

- Section 7 comparison.xls

- Taylor soil depth.xls

The directory path, sizes, and dates of the files are as follows:

Microsoft Windows 2000 [Version 5.00.2195]

(C) Copyright 1985-2000 Microsoft Corp.

$\mathrm{H}: \mid>\mathrm{D}$ :

D: $\mid>$ dir

Volume in drive D is 060706_1824

Volume Serial Number is D6EC-D29B

Directory of D:\

05/19/2006 11:08a

06/30/2006 06:04p

03/08/2006 09:55a

$06 / 29 / 2006 \quad 02: 47 \mathrm{p}$

05/26/2006 04:32p

5 File(s)

$0 \operatorname{Dir}(\mathrm{s})$
12,540,928 Depth eq. test.xls

350,208 Final calcs. for report.xls

18,432 Guertal Test.xls

1,597,952 Section 7 comparison.xls

122,368 Taylor soil depth.xls

$14,629,888$ bytes

0 bytes free

D:|> 